\title{
Alternative Fuels and Chemicals From Synthesis Gas
}

\author{
Quarterly Report \\ July 1 - September 30, 1995 \\ RECEIVED \\ NOV 161998 \\ OSTI
}

Work Performed Under Contract No.: DE-FC22-95PC93052

For

U.S. Department of Energy

Office of Fossil Energy

Federal Energy Technology Center

P.O. Box 880

Morgantown, West Virginia 26507-0880

By

Air Products and Chemicals Inc.

7201 Hamilton Boulevard

Allentown, Pennsylvania 18195-1501 


\section{Disclaimer}

This report was prepared as an account of work sponsored by an agency of the United States Government. Neither the United States Government nor any agency thereof, nor any of their employees, makes any warranty, express or implied, or assumes any legal liability or responsibility for the accuracy, completeness, or usefulness of any information, apparatus, product, or process disclosed, or represents that its use would not infringe privately owed rights. Reference herein to any specific commercial product, process, or service by trade name, trademark, manufacturer, or otherwise does not necessarily constitute or imply its endorsement, recommendation, or favoring by the United States Government or any agency thereof. The views and opinions of authors expressed herein do not necessarily state or reflect those of the United States Government or any agency thereof. 


\section{DISCLAIMER}

Portions of this document may be illegible in electronic image products. Images are produced from the best available original document. 


\title{
Alternative Fuels and Chemicals from Synthesis Gas
}

\author{
Quarterly Technical Progress Report
}

1 July - 30 September 1995

\section{CONTRACT OBJECTIVES}

The overall objectives of this program are to investigate potential technologies for the conversion of synthesis gas to oxygenated and hydrocarbon fuels and industrial chemicals, and to demonstrate the most promising technologies at DOE's LaPorte, Texas, Slurry Phase Alternative Fuels Development Unit (AFDU). The program will involve a continuation of the work performed under the Alternative Fuels from Coal-Derived Synthesis Gas Program and will draw upon information and technologies generated in parallel current and future DOE-funded contracts.

\section{SUMMARY OF ACTIVITY}

- A two-day meeting was held with the DOE and subcontractors to kick off the Alternative Fuels II and Slurry Hydrodynamics programs. Preliminary results from the June run at LaPorte were presented. Discussions were lengthy and valuable.

- Further analysis was conducted on the results from the LaPorte Hydrodynamic run in June. In particular, the gas holdup estimates were investigated, as there appeared to be a systematic difference between gas holdup based on differential pressure (DP) measurements and nuclear density gauge (NDG) readings. DP transmitters were calibrated by filling the reactor with water. The calibrations resulted in only minor corrections in the zero and the span. The NDG-based gas holdup remains 15-20\% higher than the DP-based holdup. Holdup based on shutdown tests conducted at three different conditions match well with the DP-based holdups. Discussions with Sandia National Lab personnel revealed that the NDG measurements will typically show higher holdup if a radial profile exists with higher holdup in the center. This occurs because NDG measurements weigh all the sections equally along the path of the radiation. For better accuracy, the sections near the center need lower weight since the amount of slurry with that holdup is smaller than at the sections near the wall. Typically, a radial profile for the holdup shows higher holdup in the center. This is expected to be even more prominent at high velocities studied in this run. An additional artifact could be the extra heat exchanger tubes in the annulus of the reactor, imposing further radial variations. 
- A list of targets and objectives was received from Shell for a two-month (October-November 1996) run using their cobalt catalyst. Work has begun to determine design modifications needed to achieve the goals. Shell has agreed to reduce per-pass conversion to $40 \%$. This will require connecting the F-T train to the oxygenate recycle compressor. In addition, proper degassing and possibly stripping of the reactor slurry will be required for filtration at lower temperature. Mass balance information at operating conditions (to be defined) is needed to put together an air emission permit exemption application. Mass balance information is also needed to develop the flow scheme. Information on cross-flow filtration flux is needed to design the filter loop.

- A confidentiality agreement was signed between Air Products and Shell for the FischerTropsch III test run at LaPorte. Proprietary information exchange and related process work can now go forward for the October-November 1996 run. A meeting with Shell personnel is planned in late October.

- In LPDMETM studies, the bifunctional single particle DME catalyst patented by Shell deactivated rapidly in both slurry and gas phase reactions. The slurry run was a repeat of one conducted earlier that showed the same effect.

- Tetraglyme, a hydrophobic ether, is an unsuitable slurry fluid for DME service due to its reactivity with alumina at reaction temperatures.

- Encapsulation of alumina with $\mathrm{SiO}_{2}$ did not increase $\mathrm{LPDME}^{\mathrm{TM}}$ catalyst life. Fracture of the alumina particles is the most likely cause of this behavior.

- In LPDME ${ }^{\mathrm{TM}}$ studies, modified Catapal B $\delta$-alumina, zinc exchanged $\mathrm{Y}$, and silicon ammonia phosphate were tested for use as the dehydration catalyst. Modification of the $\delta$-alumina with did not help with the stability of the catalyst system. The latter two samples exhibited nil activity. Although the silicon ammonia phosphate sample was inert in the LPDMETM run, fast long-term deactivation of the methanol catalyst was observed in this catalyst system.

- As reported by others, DME life data involving a CuO-modified alumina dehydration catalyst were inconclusive because of (unreported) mass transfer limitation in the testing apparatus. The life run was repeated under the conditions free of mass transfer limitations in our 300-cc autoclave using our version of this catalyst. The results show that the catalyst system deactivated at a rate similar to our standard dual catalyst system.

- Baseline LPDMETM tests using a copper-lined reactor for both MEOH and DME synthesis exhibited the same behavior as seen for the normal reactor. Thus, abrasion of the stainless steel walls was ruled out as a possible source of the faster aging seen in the laboratory reactors compared with the plant.

- At Aachen, a second plug flow reactor has been calibrated. Mass transfer limitations in high temperature CSTR are being studied. Isobutanol catalyst preparation using sol gel techniques continues and should be finished by next month. Undecanol is not stable enough for use as 
an inert slurry medium at high temperatures. Theoretical studies indicate that mass transfer should not be a problem in the new slurry reactor; however, unusual pressure behavior continues. The fixed bed reactor is being operated and mass balances are being verified.

- At Lehigh University, the undoped $\mathrm{CuO} / \mathrm{ZrO}_{2}$ catalyst that was first reported in the May monthly report was tested further for reproducible catalytic behavior in $\mathrm{H}_{2} / \mathrm{CO}=70.30 \%$ with and without $\mathrm{CO}_{2}$. The co-production of dimethyl ether during methanol synthesis may be controlled by varying the mol $\% \mathrm{CO}_{2}$ in the synthesis gas.

- The previously tested $\mathrm{Cu} / \mathrm{ZrO}_{2}$ catalyst $(10 / 90 \mathrm{~mol} \%)$ for alcohol/DME synthesis was tested again, except that water was added to the $\mathrm{H}_{2} / \mathrm{CO}\left(70 / 30\right.$ vol \%) feed. At $250^{\circ} \mathrm{C}$ and 1100 psi with an initial GHSV of 6120 , the methanol productivity decreased by $83 \%$, DME productivity stopped, and $\mathrm{CO}_{2}$ productivity increased by over $300 \%$.

- In work at Delaware, the final washing procedure, for example, an acetone final wash, affects catalyst performance. Contrary to results in the factorial experiment, varying copper level had little effect on performance. Interaction between $\mathrm{MnO} / \mathrm{CuO}$ and $\mathrm{ZrO}_{2} / \mathrm{CuO}$ ratios or operation at equilibrium are given as two possible explanations for this difference. Interestingly, the ability to reduce copper may be an advantage at high temperatures. (Yields continue to be about $19 \mathrm{~g} / \mathrm{IBOH} / \mathrm{Kg}$-hr @ GHSV =2900/hr, $1000 \mathrm{psi}, 400^{\circ} \mathrm{C}$ ).

- During September, Air Products compared the catalytic activity of a polymer-supported, covalently bound $\mathrm{Rh}$ complex (USP5371274) to an ionically bound $\mathrm{Rh}$ on Reillex for the $\mathrm{MeOAc}$ to EDA conversion at $150^{\circ} \mathrm{C}$. The covalently bound complex showed a higher activity in the presence of 3-picoline as a promoter. Although the ionically bound catalyst showed a higher initial activity in the presence of 3-picoline, the activity fell rapidly with recycle due to leaching of $\mathrm{Rh}$ from the polymer. We feel that the ability of our system to operate at a higher temperature $\left(190^{\circ} \mathrm{C}\right)$ without the added promoter offers a distinctive advantage over the covalently bound system. Literature suggests that polymer-supported, covalently bound $\mathrm{Rh}$ complexes leach from the polymer with time.

- The cracking of ethylidene diacetate to vinyl acetate (VAM) has proven to be problematic in the gas phase. Liquid phase experiments suggest that equilibrium is a problem. Distillation experiments show that the equilibrium can be shifted by removing the VAM as it is produced.

- At Eastman Chemical, research has been initiated on the synthesis of acetaldehyde from syngas. The current focus is on the sequential conversion of syngas to methanol to acetic acid, followed by hydrogenation of acetic acid to acetaldehyde. A proprietary catalyst has been discovered that can convert acetic acid to acetaldehyde with selectivities of $50-80 \%$.

- At Bechtel, in Task 4.2, Commercial Applications (Mixed Alcohol Synthesis), the model for the third scenario, a stand-alone mixed alcohol synthesis (MAS) plant, was completed and is being tested. Development of a model for an alternative version of the first scenario, IGCC/MAS in a utility setting, was started. This version is based on a cycling power plant, 
optimum selectivity from the MAS plant and no production or storage of methanol for peaking fuel. The effect of MAS productivity on MTBE cost will be examined.

- At Bechtel, in Task 4.5, Syngas Generation and Cleanup, calculations were made for several IGCC/MAS scenarios for the two coal feedstocks and the three gasification technologies to bracket a range of syngas flow rates to the sulfur removal processes. Nonproprietary information was obtained on three types (chemical, physical, hybrid) of conventional regenerable solvent-based sulfur removal processes. The heat and material balance and the process flow diagram for the syngas generation and cleanup for the petroleum coke case were completed. Work continued on the trace contaminants investigation.

- Electrical utility work and ventilation work on the Field Test Laboratory are essentially complete. This project is still on time and within budget.

- A meeting was held with Reema International (now REMTECH) and DOE on August 29 to discuss Reema's interest in demonstrating its F-T process at LaPorte. Reema is a small, privately owned oil and gas company based in Denver, Colorado. Significant business and technical discussions are required before the project can be kicked off. Reema will visit Air Products in mid September. An operating window of April-June 1996 was given, assuming that we do not need significant modifications and the project can be kicked off in the October-November time frame. 


\section{RESULTS AND DISCUSSION}

\section{TASK 1: ENGINEERING AND MODIFICATIONS}

\subsection{Liquid Phase Fischer-Tropsch Demonstration on Behalf of Shell Oil}

A list of targets and objectives was received from Shell for a two month (October-November 1996) run with their cobalt catalyst. Work began to determine design modifications needed to achieve the goals. Some immediate issues needed resolution before further evaluations could be made: (1) It appeared that at high conversion $(-80 \%)$ and high pressure $(750 \mathrm{psig})$, the dew point for water $\left(-235^{\circ} \mathrm{C}\right)$ is very close to the operating temperature $\left(240-250^{\circ} \mathrm{C}\right)$. The heat exchanger tubes would be significantly colder $\left(170^{\circ} \mathrm{C}\right)$ and the target temperature for filtration was $150^{\circ} \mathrm{C}$. (2) Mass balance information at operating conditions (to be defined) is needed to put together an air emission permit exemption application. It is a long lead item that needs immediate attention. Mass balance information is also needed to develop the flow scheme. (3) Information on crossflow filtration flux is needed to design the filter loop.

Response from Shell on issues was limited, pending signing of confidentiality agreements. Shell agreed to reduce per-pass conversion to $40 \%$ with a recycle in order to avoid water condensation in the reactor. This will require connecting the Fischer-Tropsch (F-T) train to the oxygenate recycle compressor. In addition, proper degassing and possible stripping of the reactor slurry will be required for filtration at lower temperature.

At the end of September, a confidentiality agreement was signed between Air Products and Shell for the F-T III test run at LaPorte. Proprietary information exchange and related process work can now go forward for the October-November 1996 run. A meeting with Shell personnel is planned in late October, followed by an update with DOE.

Tasks 1.2, 1.3, and 1.4 No progress to report this quarter.

\section{TASK 2: AFDU SHAKEDOWN, OPERATIONS, DEACTIVATION AND DISPOSAL}

\subsection{Liquid Phase Hydrodynamic Run}

Further analysis was conducted on the results from the "Hydrodynamic" run in June. In particular, the gas holdup estimates were investigated, as there appeared to be a systematic difference between gas holdup based on differential pressure (DP) measurements and nuclear density gauge (NDG) readings. DP transmitters were calibrated by filling the reactor with water. The calibrations resulted in only minor corrections in the zero and the span. The NDG-based gas holdup remains 15-20\% higher than the DP based holdup (see Figures 2.1 and 2.2). Holdups based on shutdown tests conducted at three different conditions match well with the DP-based holdups. In addition, estimated holdups from correlations based on NDG data at low velocities have a better match with holdups from DP data. Discussions with Sandia National Lab personnel revealed that the NDG measurements will typically show higher holdups if a radial profile exists with higher holdups in the center. This occurs because NDG measurements weigh all the sections equally along the path of the radiation. For better accuracy, the sections near the center 


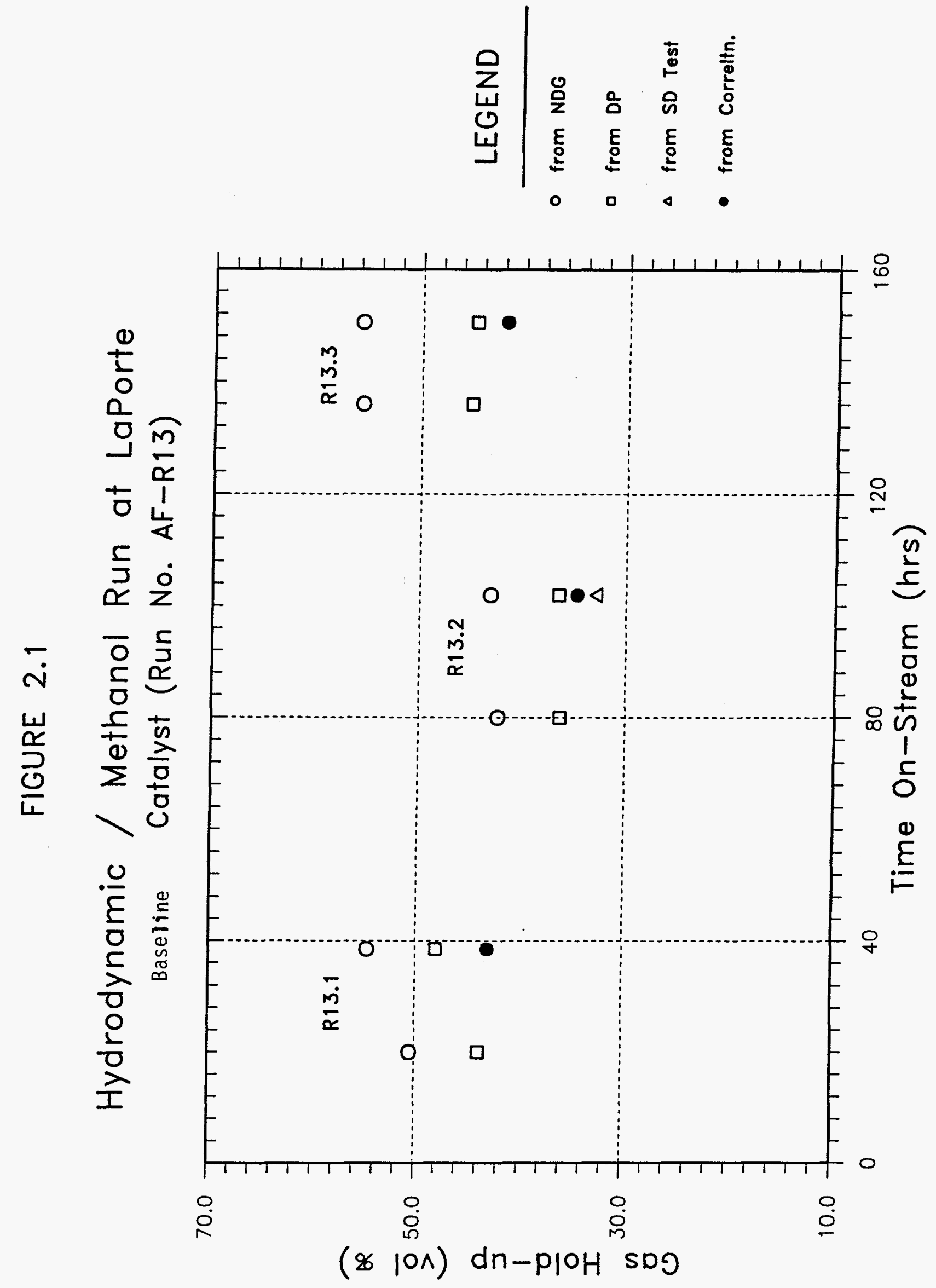




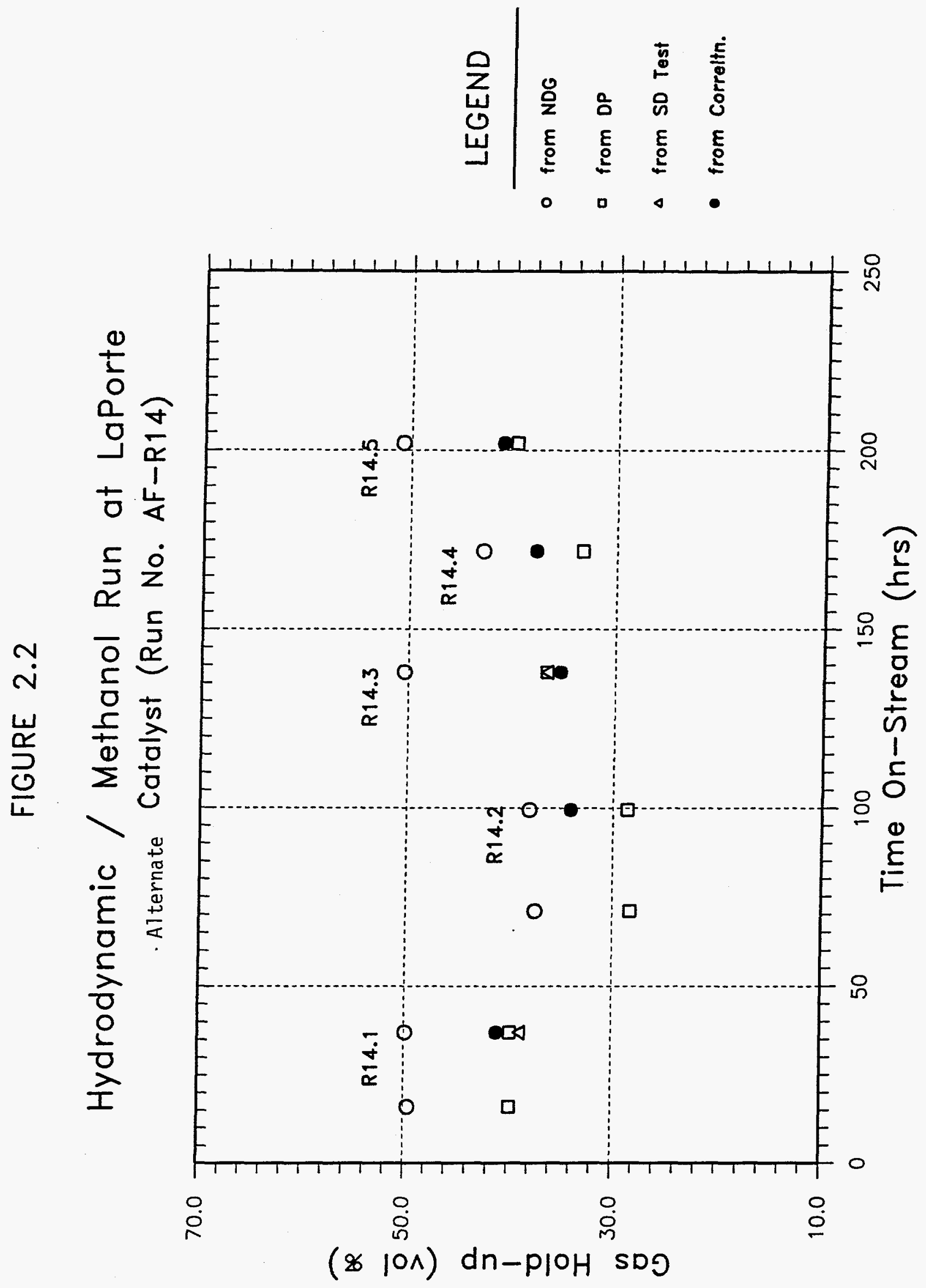


need a lower weight since the amount of slurry with that holdup is smaller than that of the sections near the wall. Typically, a radial profile for holdup shows higher holdup in the center. This is expected to be even more prominent at the high velocities studied in this run. An additional artifact could be the extra heat exchanger tubes in the annulus of the reactor, imposing further radial variations.

\section{TASK 3: RESEARCH AND DEVELOPMENT}

\section{Task 3.1 Improved Processes for DME}

This quarter's work concentrated on the screening of alternative LPDMETM catalysts. The candidates were chosen from the following categories based on our current understanding of catalyst deactivation:

- Chemically modified alumina to reduce the driving force of the interaction between methanol and dehydration catalysts.

- Physically modified alumina to eliminate the contact between the two catalysts.

- Other solid materials of moderate acidity.

- Alternative methanol catalysts such as single catalyst systems.

Most catalysts tested exhibited poorer stability than the standard dual catalyst system (S3-86 plus Catapal B $\delta$-alumina). One catalyst system exhibited improved long-term stability of the methanol catalyst.

A Japanese laboratory has recently reported a catalyst system that is stable under LPDMETM conditions. However, the life data are not reliable, or are at least inconclusive, since our analysis of the article showed that the life-test experimental conditions were subject to severe mass transfer limitations. We repeated the life run under the conditions free of mass transfer limitations. The results show that the catalyst system deactivates at a rate similar to our standard dual catalyst system.

Efforts were also made to understand the nature of the detrimental interaction between the methanol synthesis and dehydration catalysts under LPDME ${ }^{\top M}$ conditions. SEM (scanning electron microscopy) and EDS (energy dispersive X-ray spectrometry) analysis of different catalyst systems were used to determine if deactivation is due to the migration of zinc and copper from the methanol catalyst to the dehydration catalyst (e.g., $\delta$-alumina). Some of the results indicate that migration of zinc and copper from the methanol catalyst to alumina occurred under the reaction conditions. However, other inconsistent observations call for more complementary analyses before a definite conclusion can be made. 


\subsubsection{Screening of Alternative Catalyst Systems}

\subsection{1(i) $\delta$-alumina}

In the last quarterly we reported a dual catalyst system in which improved long-term stability of the methanol catalyst under LPDME ${ }^{\top M}$ conditions was observed for the first time. The dehydration catalyst in that system is a $y$-alumina heavily doped with potassium. Improved longterm stability of the methanol catalyst was observed again this quarter when a $\delta$-alumina (Condea Puralox scca-5/90) was used along with the S3-86 methanol catalyst. The run was carried out under the standard reaction conditions $\left(250^{\circ} \mathrm{C}, 750 \mathrm{psig}, 6,000 \mathrm{GHSV}, 80: 20\right.$ catalyst ratio) using Texaco gas. The results from this run are depicted in Figures 3.1.1a \& b, along with those from the catalyst systems containing the K-doped $\gamma$-alumina and virgin $\gamma$-alumina (e.g., standard system). As can be seen in Figure 3.1.1a, the long-term stability of the methanol catalyst in this catalyst system is improved.

Currently, we are trying to understand why the long-term stability of the methanol catalyst is improved in this system and in the system that contains the K-doped $\gamma$-alumina. As shown in Figure 3.1.1b, the dehydration activity of both $\delta$-alumina and $\mathrm{K}$-doped $\gamma$-alumina are very much lower $\left(\mathrm{k}_{\mathrm{d}} \leq 3.0\right.$ and 1.3 , respectively) than that of $\gamma$-alumina. We have tested systems with similar and poorer dehydration activity, such as those using Chabazite $\left(\mathrm{k}_{\mathrm{d}} \leq 1.3\right), \mathrm{Mg}-\mathrm{Y}\left(\mathrm{k}_{\mathrm{d}} \leq\right.$ 0.9 ), and a silica alumina sample $\left(\mathrm{k}_{\mathrm{d}} \leq 0.6\right)$ as dehydration catalysts (see April-June quarterly). However, the long-term deactivation of the methanol catalyst in these systems is similar to the relatively rapid deactivation of the standard system. This indicates that lower dehydration activity does not guarantee better long-term stability of the methanol catalyst. What we would like to understand is whether the low dehydration activity is a necessary condition for the longterm stability of the methanol catalyst. If it is, we should look for weakly acidic materials with high site density as dehydration catalysts, since it is assumed that the low activity seen in these two systems is related to the weak acid sites. If low activity is not a necessary condition, we need to understand what is special about these two systems so that screening guidelines can be developed for alternative dehydration catalysts.

\subsection{1(ii) Modified $\gamma$-alumina}

Three modified Catapal B $\gamma$-alumina catalysts have been tested. The modification methods include impregnation using potassium, silylation using $\left(\mathrm{Me}_{3} \mathrm{Si}-\right)_{2} \mathrm{NH}$, and encapsulation with $\mathrm{SiO}_{2}$. The potassium-impregnated sample was prepared by the incipient wetness method using a $\mathrm{KOH}$ aqueous solution. This sample had higher dehydration activity due to a lower $\mathrm{K}$ loading than the K-doped sample discussed in section 3.1.1(i) ( $0.15 \mathrm{vs.} 0.8 \mathrm{wt} \%$ of $\mathrm{K})$. The goal of this new preparation was to determine if the improved long-term stability of the methanol catalyst seen in the sample with $0.8 \mathrm{wt} \% \mathrm{~K}$ loading could still be retained with a more active $\mathrm{K}$-doped alumina. Silylation using ( $\mathrm{Me}_{3} \mathrm{Si}-\mathrm{NHH}_{\mathrm{N}}$ was aimed at removing the strong acid sites on Catapal $\mathrm{B}$ $\gamma$-alumina. The sample was prepared by refluxing Catapal B $\gamma$-alumina in $\left(\mathrm{Me}_{3} \mathrm{Si}-{ }_{2} \mathrm{NH}\right.$. If an inert, porous layer of $\mathrm{SiO}_{2}$ can be formed on the outside of alumina particles, intimate contact between the alumina and the methanol catalyst should be prevented. This, in turn, should lead to good stability of the catalyst system. The encapsulation of the alumina with $\mathrm{SiO}_{2}$ was carried out according to a DuPont patent (US Patent 4,677,084). The LPDME ${ }^{\mathrm{TM}}$ runs using these three 
Figure 3.1.1 LPDME ${ }^{T M}$ Life Runs of Different Catalyst Systems
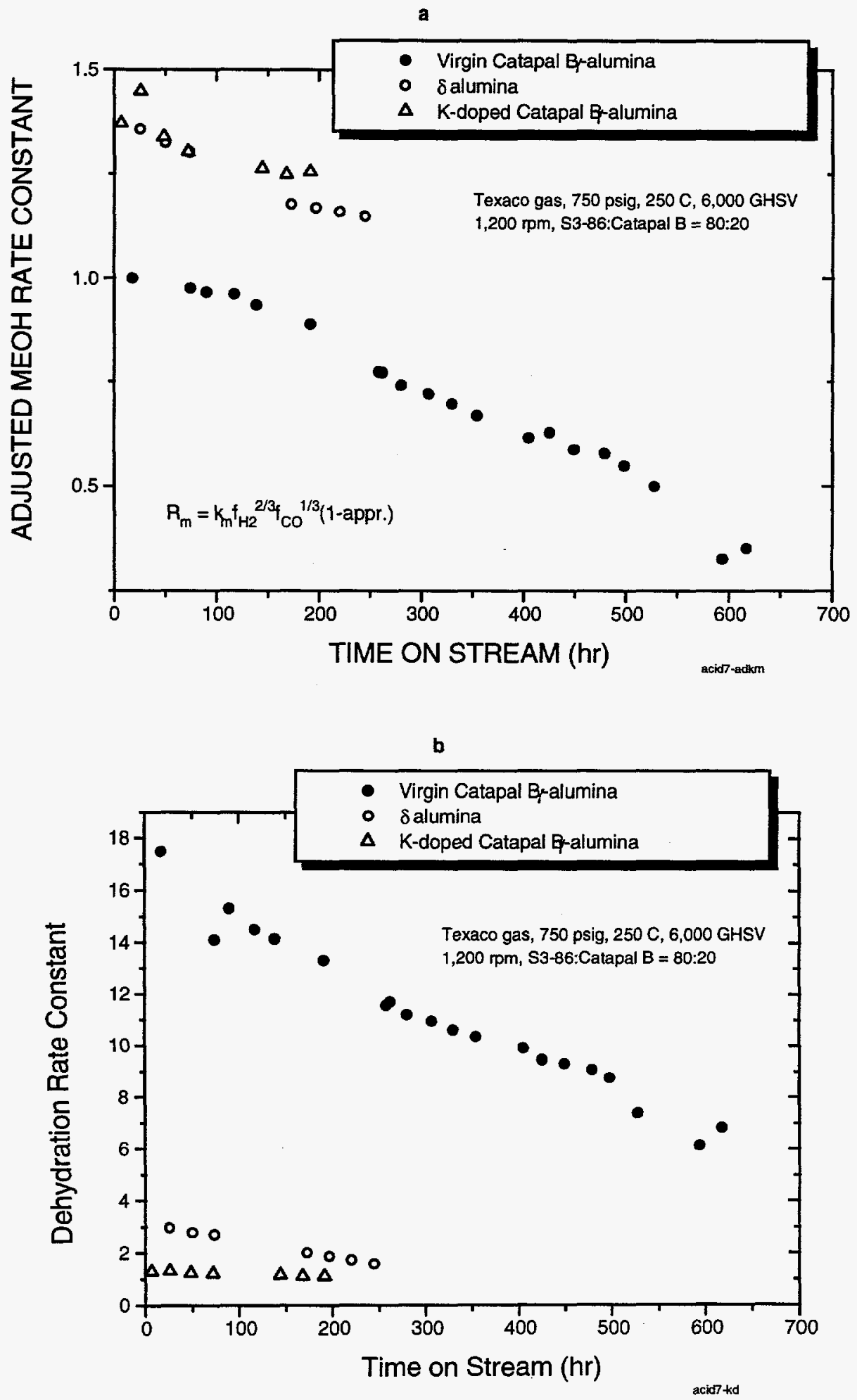
dehydration catalysts were all conducted under the standard reaction conditions using BASF S3-86 as the methanol synthesis catalysts. The results are plotted in Figures 3.1.2a and b against those from the standard catalyst system.

In contrast to the previous result obtained using $0.8 \mathrm{wt} \% \mathrm{~K}$ loading, doping the alumina with $0.15 \mathrm{wt} \%$ of $\mathrm{K}$ did not result in better long-term stability of the methanol catalyst. Figures 3.1.2a and $b$ show that the long-term stability of both methanol synthesis and dehydration catalysts in this system is poorer than that of the standard dual catalyst system. We do not understand why the potassium doping at different levels leads to different effects on the long-term stability of the catalyst system. The effect cannot be attributed to the acidity of the dehydration catalyst because the dehydration activity of the $0.5 \mathrm{wt} \%$ sample $\left(\mathrm{k}_{\mathrm{d}}=8.5\right)$ lies between the virgin alumina $\left(\mathrm{k}_{\mathrm{d}}=\right.$ $17.5)$ and the $0.8 \mathrm{wt} \%$ sample $\left(\mathrm{k}_{\mathrm{d}}=1.3\right)$.

Figures 3.1.2a and $\mathrm{b}$ shows that silylation of Catapal B $\gamma$-alumina with $\left(\mathrm{Me}_{3} \mathrm{Si}-\right)_{2} \mathrm{NH}$ does not result in better long-term stability. The initial deactivation of the methanol catalyst in this system is also greater than that in the standard system. Since silylation in general is believed to passivate the strong acid sites on acidic solid materials, this greater initial deactivation is probably due to the poisonous species in the $\left(\mathrm{Me}_{3} \mathrm{Si}-\right)_{2} \mathrm{NH}$-modified alumina.

The $\mathrm{SiO}_{2}$-encapsulated alumina sample was analyzed prior to the run by scanning electron microscope (SEM) and energy dispersive X-ray spectrometry (EDS). The results show that most of the silica, as desired, was on the exterior of the alumina particles. However, this sample did not bring about the better stability we expected. As shown in Figures 3.1.2a \& b, the stability of this system is poorer than the standard catalyst system; both methanol and dehydration catalysts deactivated very rapidly. Another run (not shown) using the same sample calcined at a higher temperature yielded a similar result. The poor stability may have been due to the poor mechanical strength of the encapsulated alumina particles. Evidence of this hypothesis is the observation that during the SEM/EDS experiments the preparation of the cross section of alumina particles was much more difficult compared to virgin Catapal B $\gamma$-alumina because the particles broke apart readily. The SEM cross section of an alumina particle also appeared flaky. The exposure of the alumina to a fairly strong acidic environment $(\mathrm{pH}=2)$ during the encapsulation may be the reason for the lower strength. If the particles cannot retain their integrity, a protective layer of $\mathrm{SiO}_{2}$ will be useless. The cleavage of the particles under the reaction conditions (strong agitation) will create an active alumina surface, which then interacts with the methanol catalyst to cause the deactivation of both catalysts. This issue will be examined in greater depth, and further efforts will be made to prepare encapsulated alumina samples.

\subsubsection{9(iii) Other Acidic Materials}

Two samples we tested in this category are silica ammonia phosphate and zinc ion-exchanged zeolite $\mathrm{Y}(\mathrm{Zn}-\mathrm{Y})$. The $\mathrm{LPDME}^{\mathrm{TM}}$ run using the silicon ammonium phosphate sample as the dehydration catalyst was conducted under the standard conditions $\left(250^{\circ} \mathrm{C}, 750 \mathrm{psig}, 6,000\right.$ GHSV) using Texaco gas. It has been shown in Air Products' laboratory that the activity of this sample toward isobutanol dehydration at $300^{\circ} \mathrm{C}$ is similar to $\gamma$-alumina. Surprisingly, little 
Figure 3.1.2 The Stability of Different Catalyst Systems under LPDME ${ }^{\mathrm{TM}}$ Conditions
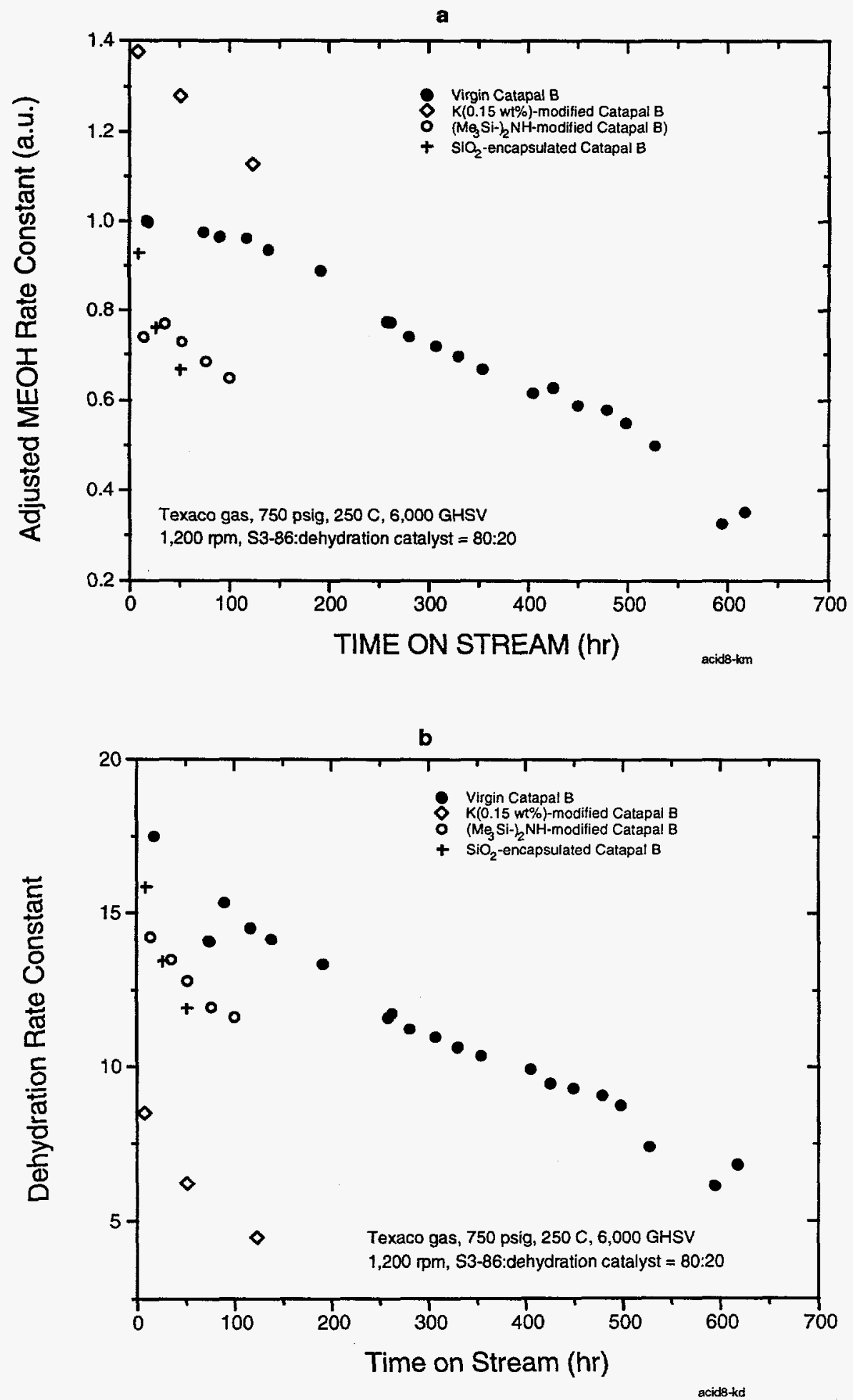
dehydration activity was detected in the LPDME ${ }^{\mathrm{TM}}$ run. Increasing the temperature to $280^{\circ} \mathrm{C}$ had little effect. An interesting observation from this run is that the long-term deactivation of the methanol catalyst in this system is faster than in the standard catalyst system (Fig. 3.1.3), even though the phosphate sample did not show any dehydration activity. This again indicates that the dehydration activity, or acidity, is not necessarily the cause for the long-term deactivation of the methanol catalyst. When the reactor was taken apart after the run, it was observed that the spent catalyst stuck to the surface of the reactor hardware more strongly than it did in the standard catalyst system.

\section{Figure 3.1.3 A LPDME ${ }^{\top м}$ Life Run Using Silicon Ammonia Phosphate as the Dehydration}

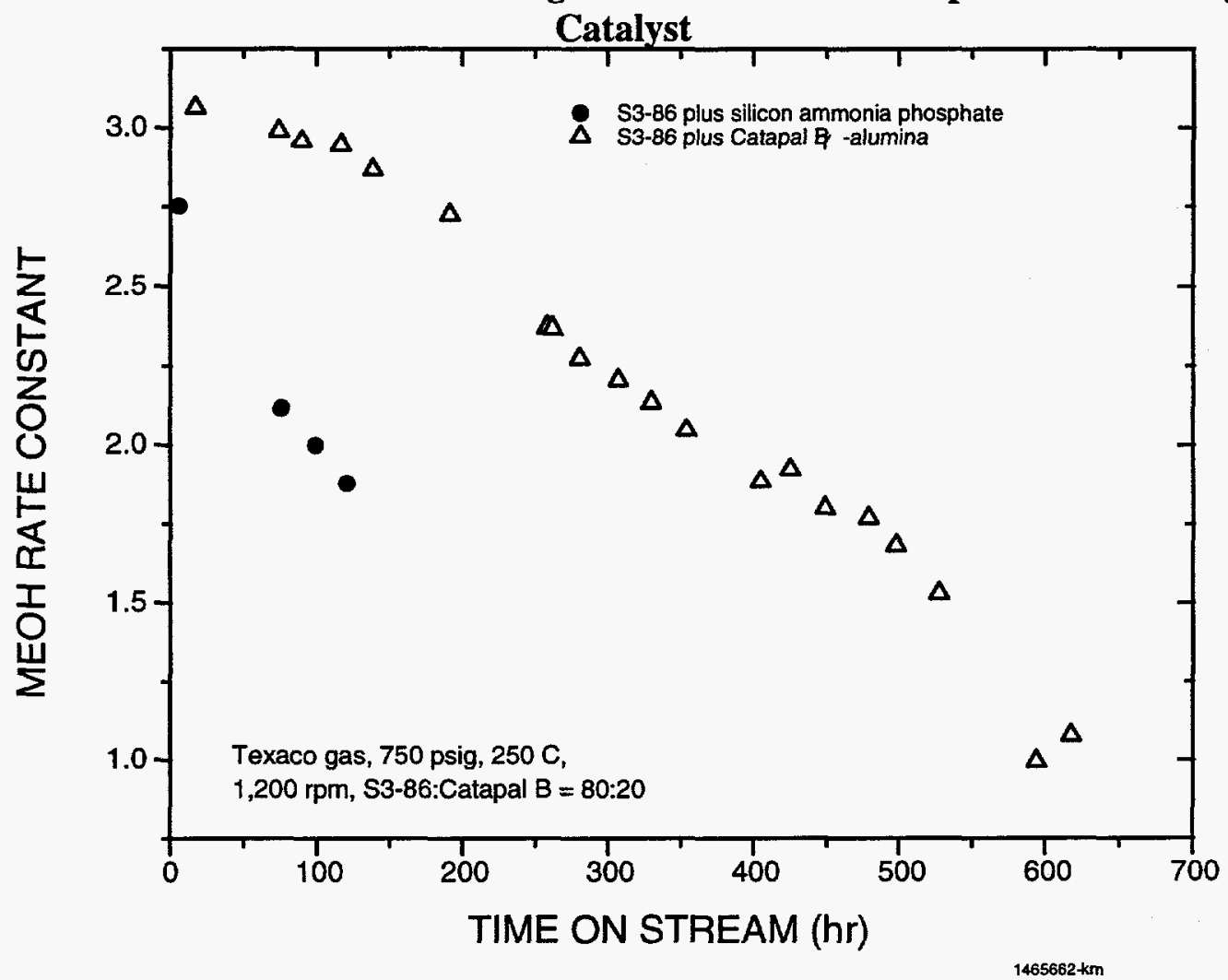

$\mathrm{Zn}-\mathrm{Y}$ was chosen on the basis of the migration hypothesis. If $\mathrm{Zn}$ migration from the methanol catalyst to the dehydration catalyst is the cause of catalyst deactivation, it is unlikely that migration will take place in a dehydration catalyst that has already been saturated with $\mathrm{Zn}$, such as $\mathrm{Zn}-\mathrm{Y}$. However, this sample did not exhibit any dehydration activity.

\subsection{1(iv) Single Catalyst System - Cu and Zn Supported on $\gamma$-Alumina}

It was reported in the April-June quarterly that a single particle catalyst system consisting of $\mathrm{Cu}$, $\mathrm{ZnO}$ supported on $\gamma$-alumina has a poorer stability under LPDME ${ }^{\mathrm{TM}}$ conditions than the standard dual catalyst system. That catalyst was prepared by impregnating Catapal B $\gamma$-alumina powders with zinc and copper nitrate followed by calcination in air, in accordance with a Shell patent (US $4,375,424,1983)$. To find out if the poor stability is intrinsic to the catalyst or related to the slurry phase operation, a similar catalyst was prepared from Catapal B $\gamma$-alumina pellets and 
tested in a packed bed reactor. The final $\mathrm{Cu}$ and $\mathrm{Zn}$ content based on an elemental analysis was 4.0 and $4.6 \mathrm{wt} \%$, respectively. The catalyst bed was diluted with quartz chips. The reduction was carried out using $2 \% \mathrm{H}_{2}$ in $\mathrm{N}_{2}$ at $700 \mathrm{psig}$ and a ramp from room temperature to $250^{\circ} \mathrm{C}$ in 3 $\mathrm{hr}$, mimicking the Shell procedures. Two packed bed runs were conducted because some equipment problems were encountered in the first run. The results from these two runs are displayed in Figure 3.1.4, along with the runs conducted in the autoclave using the single catalyst and the standard dual catalyst system.

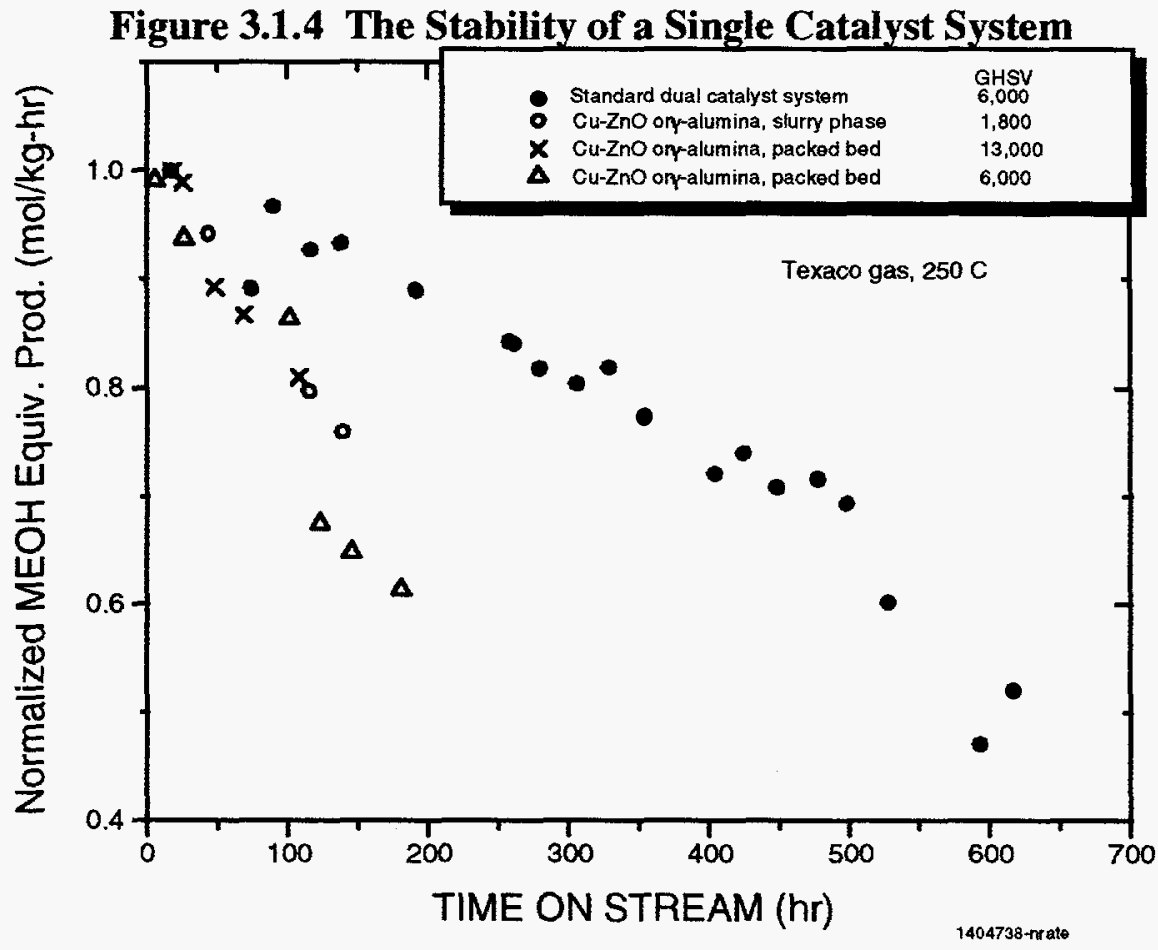

Figure 3.1.4 shows that the aging pattern of the single catalyst system in the packed bed runs is similar to that in the slurry phase run. This indicates that the poor stability is intrinsic to the catalyst sample, rather than to the slurry phase operation. In order to test the single catalyst concept, a DME catalyst that is at least stable in the gas phase operation is needed.

Several data points were collected at $270^{\circ} \mathrm{C}$ in order to compare the activity of the single catalyst system to that of the catalysts reported in the Shell patent. The results show that the catalyst made in the laboratory had about one-half of the activity of a corresponding sample in the patent. This may be partly due to $20 \%$ lower $\mathrm{Cu}$ loading and $10 \% \mathrm{Zn}$ loading in our catalyst.

\subsubsection{A Repeat Life Run of a Reported Dual Catalyst System for LPDME ${ }^{\text {TM }}$}

According to the work in a Japanese laboratory, a dual catalyst system consisting of ICI 51-2 methanol catalyst and $\mathrm{Cu}$-doped $\gamma$-alumina ( $5 \mathrm{wt} \%$ ) powders, with hexadecane as the slurry fluid, exhibited no loss in activity under LPDME ${ }^{T M}$ conditions for 700 hours (private communication). A close examination of the data reveals that the life run is suspect since the catalyst was subject to 
severe mass transfer limitations. (After our experiments were completed, the authors of the report acknowledged this problem and stated that the catalyst system may actually deactivate faster. No elaboration was offered.) We decided to examine the stability of the catalyst system in our $300 \mathrm{cc}$ autoclave under the conditions free of mass transfer limitations. A Cu-doped $\gamma$ alumina sample was prepared according to the method reported in a patent by the Japanese authors. The same gas composition $\left(50: 50 \mathrm{H}_{2}\right.$ to $\mathrm{CO}$ ), catalyst ratio, solid concentration in the slurry, and slurry fluid were used. The run was conducted first at $250^{\circ} \mathrm{C}, 750 \mathrm{psig}$, and a space velocity of $1980 \mathrm{sl} / \mathrm{kg}$-hr for $142 \mathrm{hr}$. Afterward the run was continued at $280^{\circ} \mathrm{C}, 426 \mathrm{psig}$, and $1980 \mathrm{sl} / \mathrm{kg}$-hr for $43 \mathrm{hr}$, which are the exact conditions used in the reported life study. The activity of our version of the catalyst system was similar to that in the report, but stable activity was not observed. As shown in Fig. 3.1.5, the catalyst system deactivated under both sets of conditions. The rate of deactivation is similar to our standard catalyst system (S3-86 plus Catapal $\mathrm{B} \gamma$-alumina). These results suggest that the LPDME ${ }^{\mathrm{TM}}$ run in the Japanese laboratory may also have had a stability problem, unless certain factors were not disclosed in their report and patent.

\subsubsection{SEM/EDS Analysis of LPDMETM Catalysts - Examination of the Migration Hypothesis}

\section{Summary}

This section summarizes the analytical results of methanol catalyst and alumina mixtures using SEM and EDS. The analysis was performed to confirm the hypothesis that the deactivation of the LPDME $^{T M}$ catalyst system is caused by inter-catalyst migration of one or more types of atoms. The questions to be answered by this analysis are: Does the migration take place? And if it does, is the migration correlated to the catalyst deactivation?

Zinc and copper were detected on alumina particles in the spent catalyst mixtures, but not on virgin alumina, and the ratio of copper to zinc was significantly different from that found for the methanol catalyst itself. This indicates that migration occurs, at least in some cases. Furthermore, no migration of zinc or copper was found in the Robinson-Mahoney experiment in which the catalyst did not deactivate rapidly. Unfortunately, the zinc and copper content of alumina particles in catalyst mixtures of different histories does not correlate with the activity of the catalyst systems. Several explanations for this behavior have been proposed:

1) methanol catalyst fines may exist in the alumina particles and thus, confound the analysis;

2) migration is only responsible for the initial deactivation of the catalyst system; or

3) the sampling may not be statistically sound. 


\section{Figure 3.1.5 The Stability of a Reported LPDME ${ }^{\top M}$ Catalyst System Tested in Air Products' Laboratory}

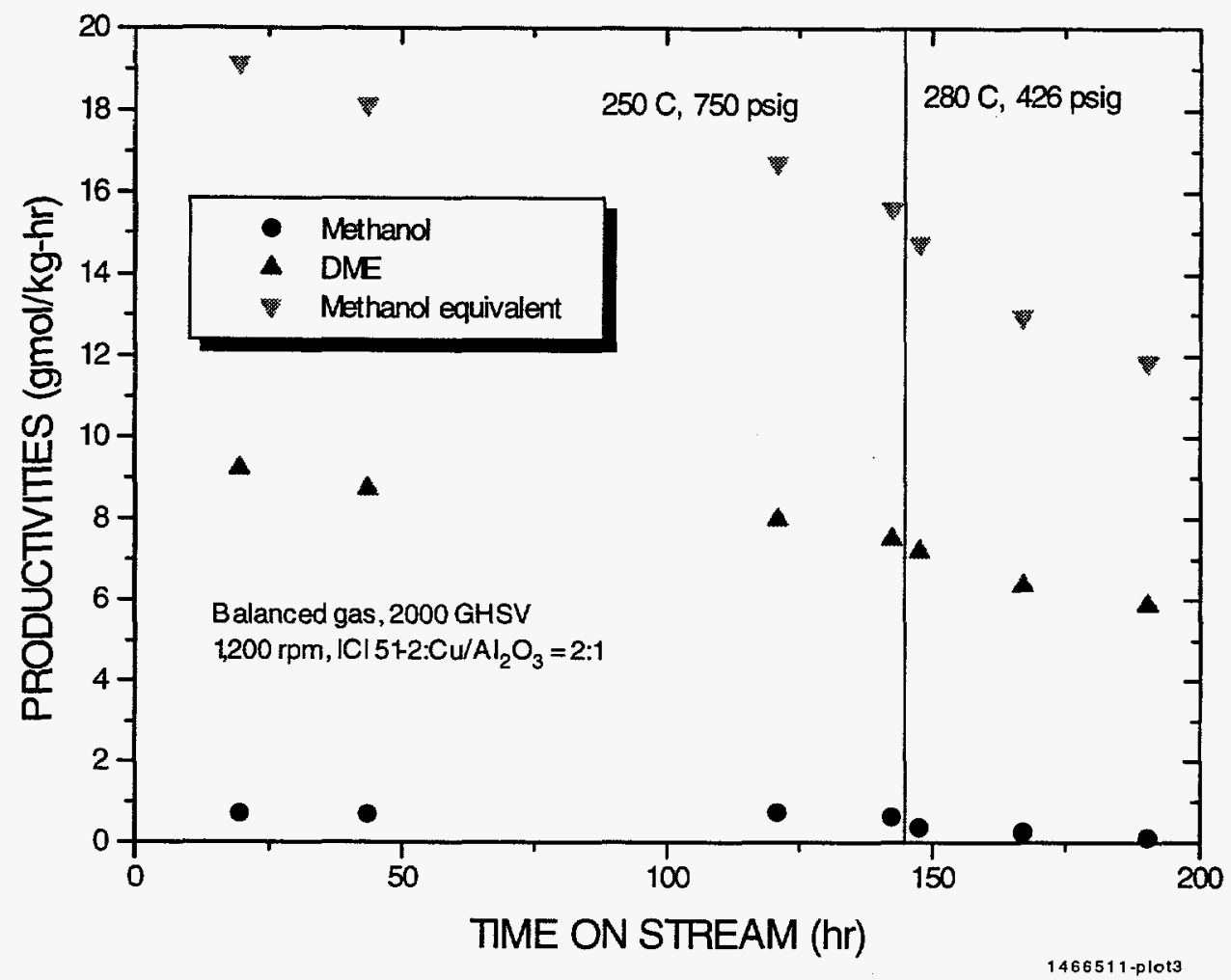

This lack of correlation is not sufficient to reject the migration of zinc and/or copper as the primary cause of catalyst deactivation. Work on understanding the mechanism of migration continues.

\section{Introduction}

We reported previously that an interaction between a methanol synthesis catalyst (e.g., BASF S3$86 \mathrm{Cu}-\mathrm{ZnO}$-based low pressure methanol catalyst) and a dehydration catalyst (e.g., $\gamma$-alumina) in the standard dual catalyst system is responsible for the deactivation of both catalysts under LPDMETM conditions. Several observations have suggested that this interaction could be due to inter-catalyst migration of zinc and copper, that is, the zinc- or/and copper-containing species from the methanol catalyst migrate onto the surface of the dehydration catalyst during reduction and/or under the reaction conditions. By this mechanism, the methanol catalyst deactivates because it loses its active components. The dehydration catalyst is poisoned by these migrating species by reacting with the acid sites. Possible driving forces for the inter-catalyst migration are:

1) acid-base reactions - solid state reactions such as acid-base reactions between $\mathrm{ZnO}$ in the methanol catalyst and the acid sites on the dehydration catalyst,

2) ion exchange - solid-state exchange of copper/zinc ions from the methanol catalyst with the protons (i.e., Bronsted acid sites) on the dehydration catalyst, 
3) spontaneous dispersion - migration simply driven by the concentration gradient of copper and zinc between the two catalysts, considering that $\gamma$-alumina, and most other types of dehydration catalysts, for that matter, have good dispersing capability toward metal, metal oxide, and salts.

These processes must take place where two catalysts contact each other, that is, at the outer surface of alumina particles. The migration may or may not continue into the inner surface of the alumina particles. However, the extent of the deactivation we have seen suggests that the inner surface of the alumina should be affected, unless the alumina particles break apart considerably under the reaction conditions. Efforts have been made to confirm the hypothesis by analyzing the copper and zinc content in the alumina in spent catalyst mixtures. This report summarizes the results based on the analysis using SEM and EDS.

\section{Method of Analysis}

SEM and EDS were used because the different types of catalyst particles are inseparable. The catalyst system under study consists of a powder mixture of the two catalysts. The mean diameter of fresh methanol catalyst and alumina powders is around 60 microns. The particle size in the spent catalyst mixture is smaller because of the attrition in the reactor. This makes it virtually impossible to separate the alumina from the methanol catalyst and use it for conventional elemental analysis. SEM enables us to pick out individual alumina or methanol catalyst particles, while EDS provides elemental information on these particles. The smallest features that can be "seen" by SEM are 0.1 to 1 micron. The spatial resolution of EDS of 1 micron, and the lower detecting limit of EDS is believed to be $0.5 \mathrm{wt} . \%$.

We looked at both the outer surface and the cross section of alumina particles. Inter-catalyst migration will initiate on the outer surface due to the contact of the two catalysts. Figure 3.1.6 is a SEM micrograph of the outer surface of an alumina particle in a spent catalyst mixture. The bright spots are methanol catalyst powders, judging by EDS analysis and the brightness of these powders. (Heavier elements like $\mathrm{Cu}$ and $\mathrm{Zn}$ have a brighter image in SEM than lighter elements like Al.) These powders are physically attached to the alumina, a phenomenon that can be inferred since treating the sample with an organic removes most of the metal particles through dispersion. As shown in Figure 3.1.7, the outer surface of an alumina particle in a catalyst mixture dispersed using an organic solvent is much cleaner, although some methanol catalyst particles can still be seen. In both non-dispersed (or dry) and dispersed samples, "clean" areas where no methanol catalyst particles are found exist as illustrated in Figures 3.1.6 and 3.1.7. The "clean" area was assumed to be free of physically attached methanol catalyst particles, and used for the analysis.

We also looked at the cross section of the alumina particles as a way to circumvent the physical attachment problem. Moreover, this indicates if the migration into the inner surface of alumina particles has occurred. The cross section was prepared by freezing the sample in epoxy and cutting it to expose the cross section. Precautions were taken to make sure that copper and zinc from the methanol catalyst were not smeared onto the alumina cross section by the cutting. This was checked by EDS examination of the epoxy around the sample particles. Little copper and zinc were detected. 
Figure 3.1.6 SEM Micrograph of an Alumina Particle in a Spent Catalyst Mixture.

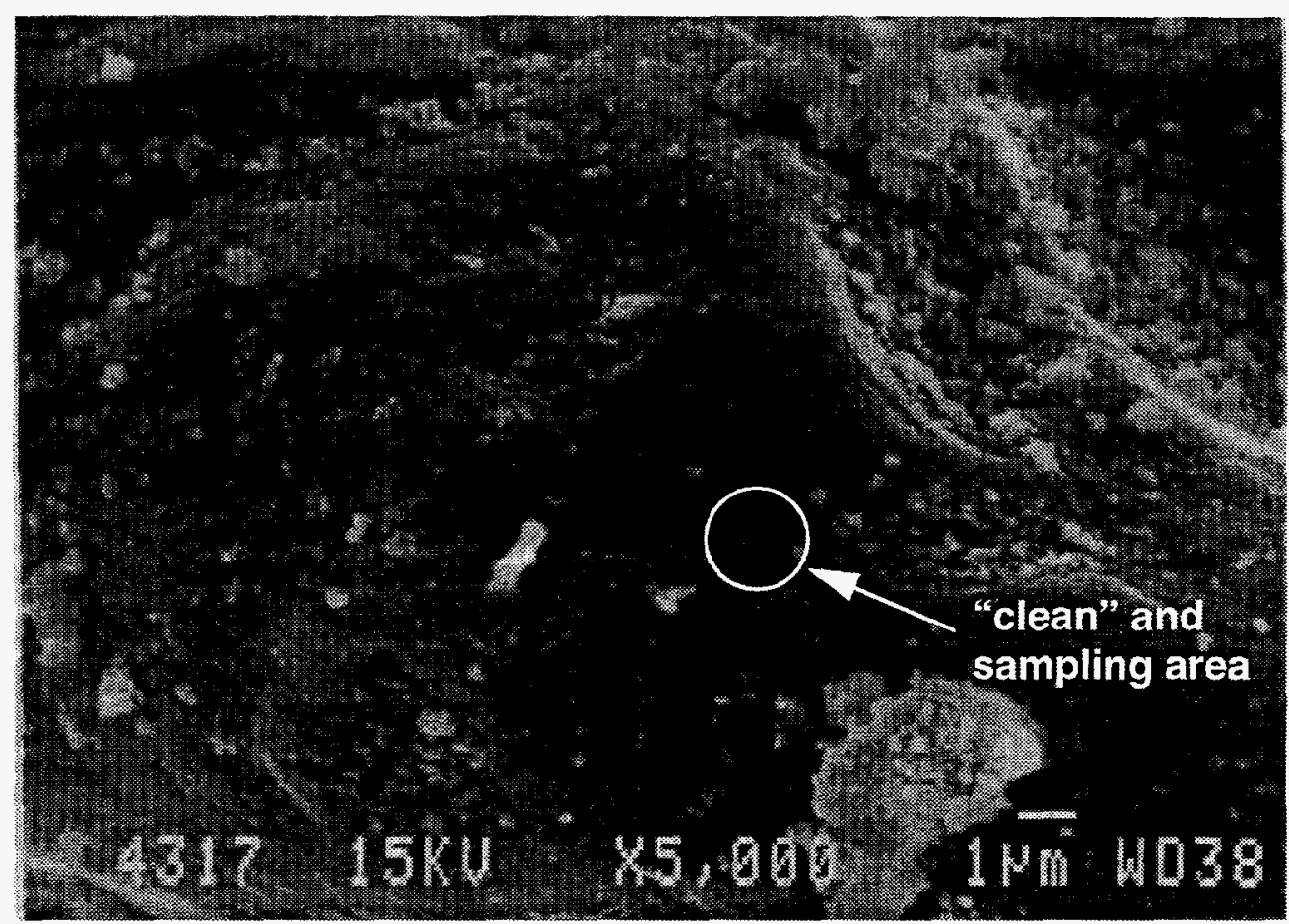

Figure 3.1.7 SEM Micrograph of an Alumina Particle in a Dispersed Spent Catalyst Mixture

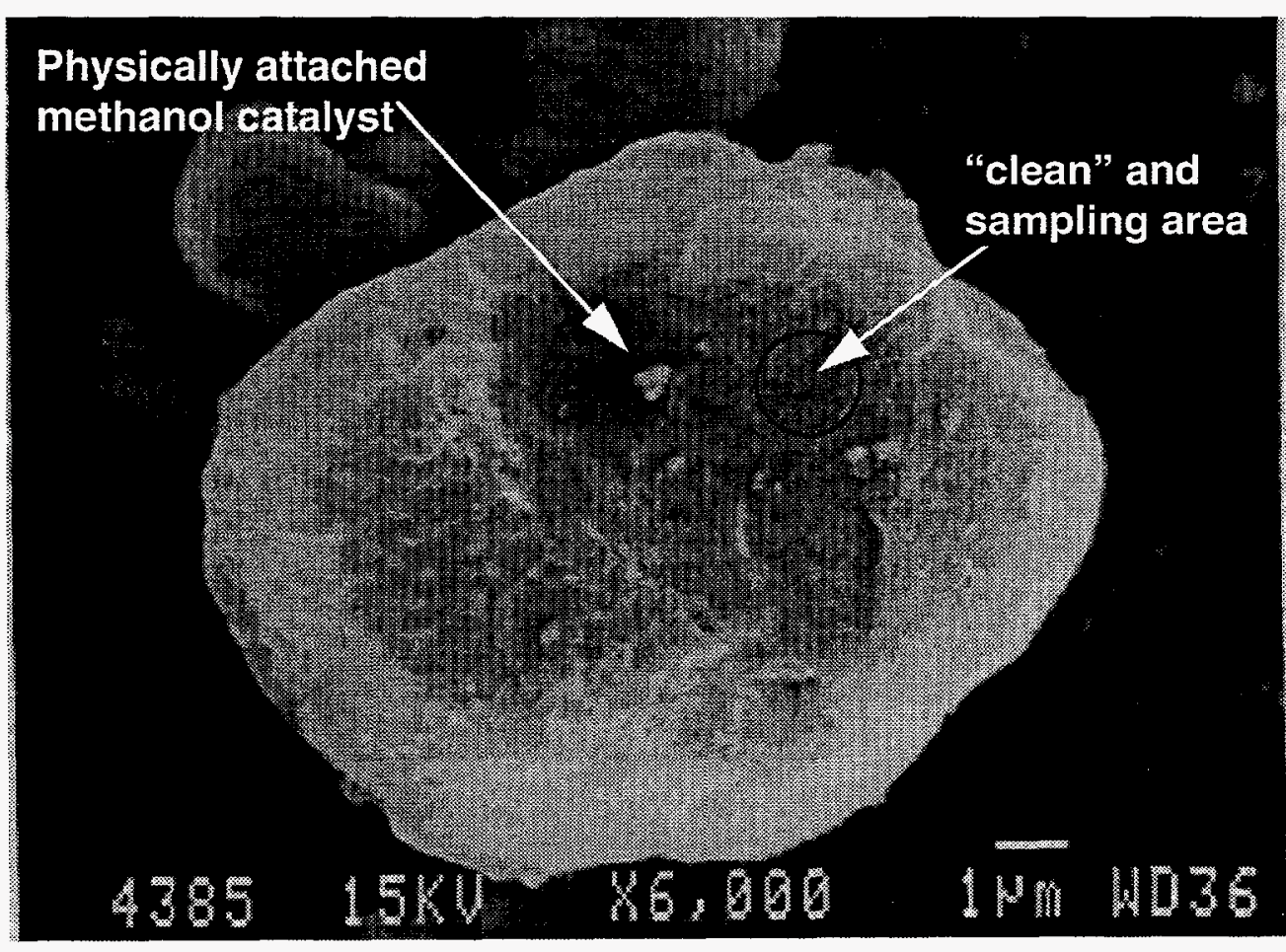

sem1.ppt 
Figure 3.1.8 illustrates how a typical analysis was conducted. An SEM micrograph of an alumina particle, a cross section in this case, was generated first. Then the electron beam was parked at different spots in this cross section, and an EDS elemental analysis was performed. The sampling volume in this mode of analysis was an order of magnitude smaller than the dimension of alumina particles. Alternatively, one could raster the beam across the sample to obtain integrated elemental information.

\section{Figure 3.1.8 SEM Micrograph of the Cross Section of an Alumina Particle in a Spent Catalyst Mixture}

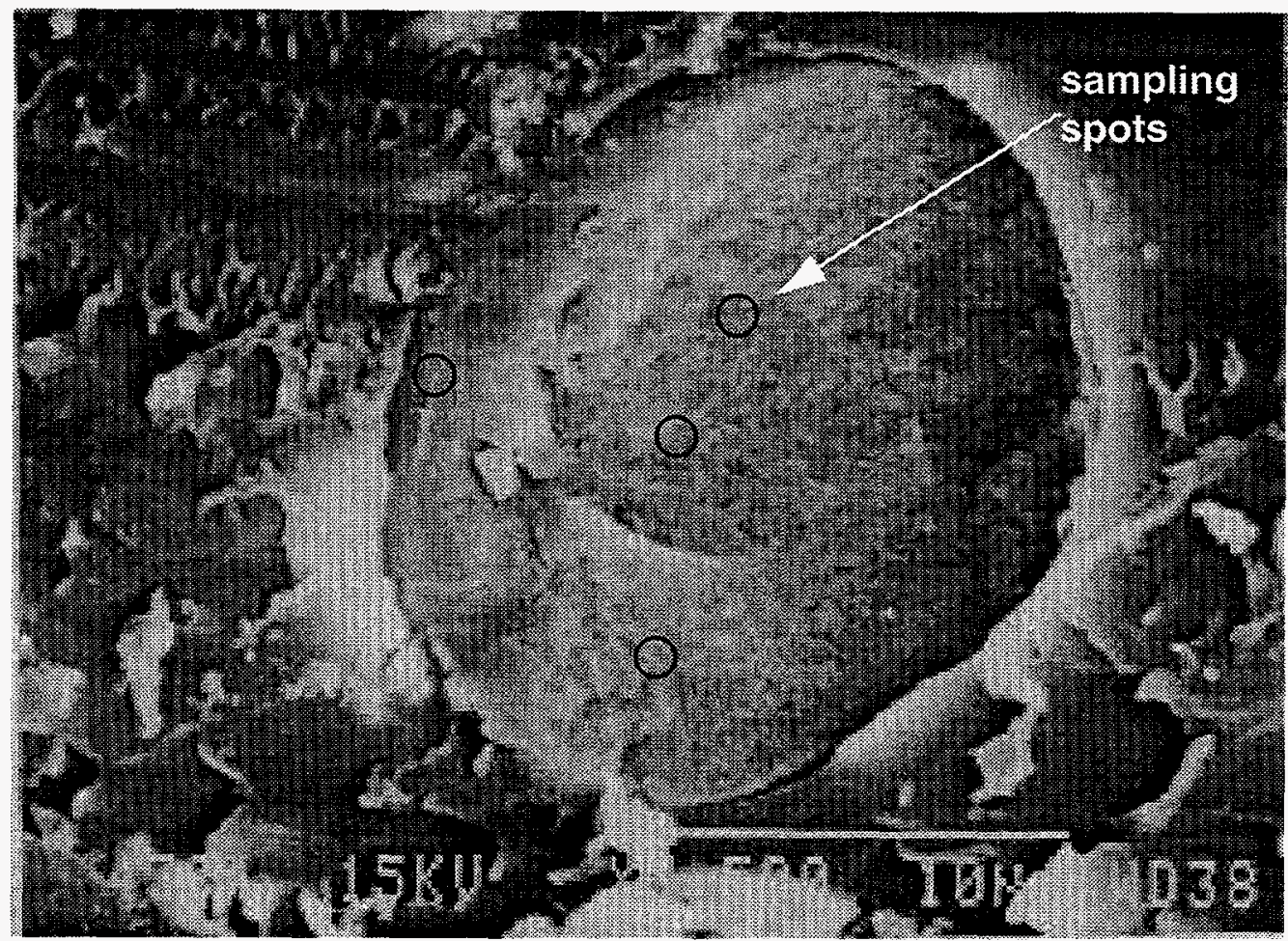

The elemental analysis by EDS is based on the energy dispersion of the X-ray generated by the same electron beam that is used for SEM. Figure 3.1.9a illustrates how different elements in a methanol catalyst sample appear on an EDS spectrum. (The aluminum section of the spectrum is not shown.) The net peak area was used for quantitative analysis. The integration was conducted using graphics software called Origin, since the data processing software used by the EDS instrument tended to draw erroneous baselines, especially when the signal was weak. To make sure the quantitation was independent of analytical conditions, such as the size of sample particles and the electron beam, gold coating, and parking mode vs. rastering mode, we looked at methanol catalyst particles of different sizes, with and without gold coating (gold coating was used to remove the static charging on the sample), and of different origins. The particles included those in a pure, freshly reduced methanol catalyst sample, in a spent catalyst mixture, and those physically attached to the outer surface of alumina particles in the same spent catalyst mixture. As shown by the solid dots in Figure 3.1.10, the $\mathrm{Zn}$-to-Cu ratio from these different samples falls into a narrow range around 0.29 , indicating that the quantitation is insensitive to the analytical conditions. 
Figure 3.1.9 EDS Spectra of Different Samples

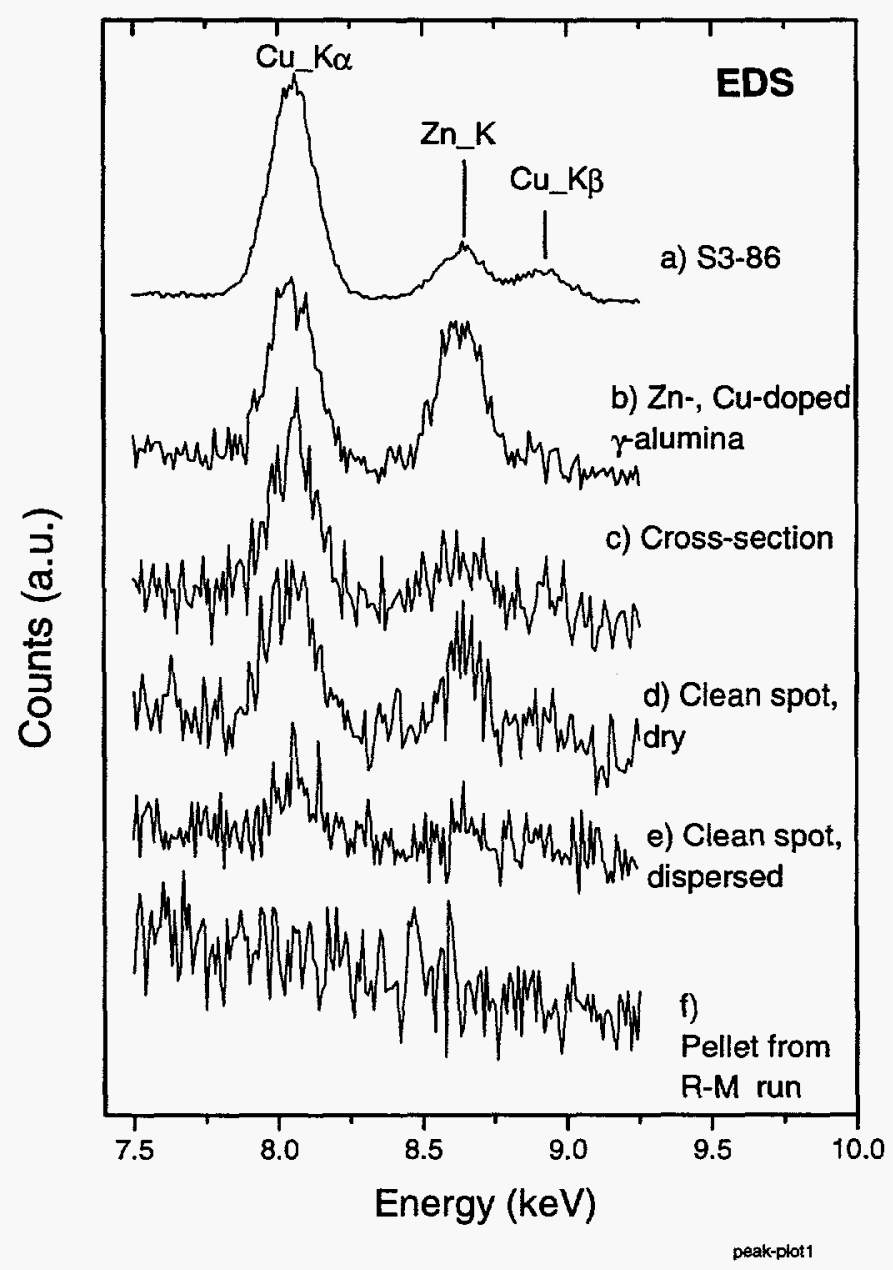


Figure 3.1.10 Zn-to-Cu EDS Peak Ratio of Different Samples

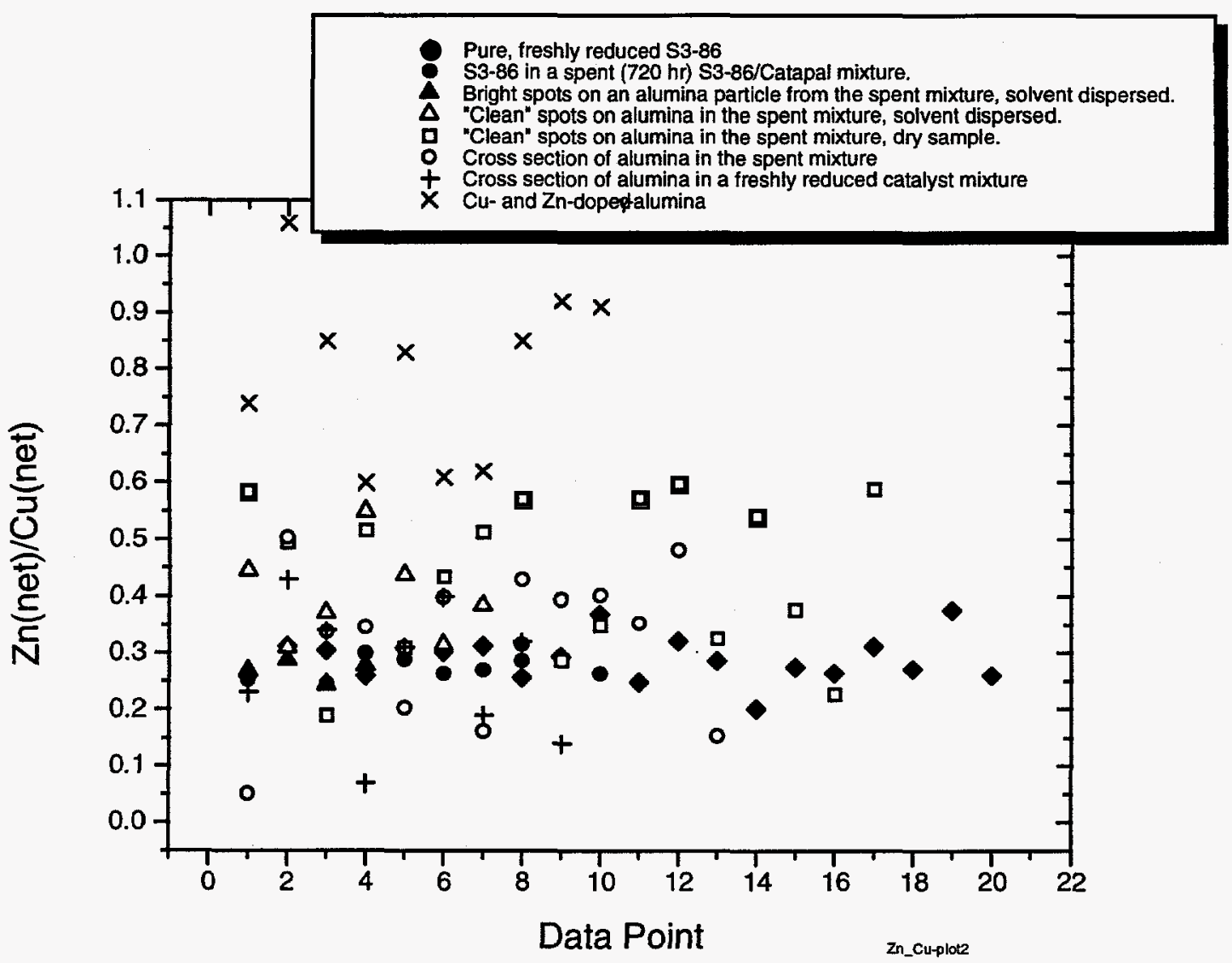

Since the absolute quantitation was difficult to measure, we relied on relative quantity of different elements for our analysis. These included ratios of $(\mathrm{Zn}+\mathrm{Cu})$ to $\mathrm{Al}$ and $\mathrm{Zn}$ to $\mathrm{Cu}$. The $(\mathrm{Zn}+\mathrm{Cu})$ to $\mathrm{Al}$ ratio is intended as a measure of how much zinc and copper migrate onto the alumina, while the $\mathrm{Zn}$ to $\mathrm{Cu}$ ratio serves as a way to distinguish migrated zinc and copper from that in the physically attached methanol catalyst particles. We assume that zinc and copper deposition on the alumina due to the migration is unlikely to have the same ratio as in the methanol catalyst.

\section{Results and Discussion}

All EDS results, that is, the net peak area of $\mathrm{Cu}, \mathrm{Zn}$, and $\mathrm{Al}$ plus their ratios, are summarized in Table 3.1.1. Figure 3.1.9 displays typical EDS spectra from different samples. The $\mathrm{Zn} / \mathrm{Cu}$ and $(\mathrm{Zn}+\mathrm{Cu}) / \mathrm{Al}$ ratios from different samples are shown in Figures 3.1.10 and 3.1.11. The three main samples discussed below are the alumina in a catalyst mixture that was on stream for 710 hours (the spent sample), the alumina in a freshly reduced catalyst mixture (the freshly reduced sample), and the alumina in a catalyst mixture used in a catalyst compatibility study (the compatibility sample). The relative activities of these three catalyst systems, in terms of the methanol equivalent productivity, are $0.6,1$, and 0.5 , respectively. The analytical results from these samples do not show a consistent picture. The data that appear to support the migration theory will be examined first. 


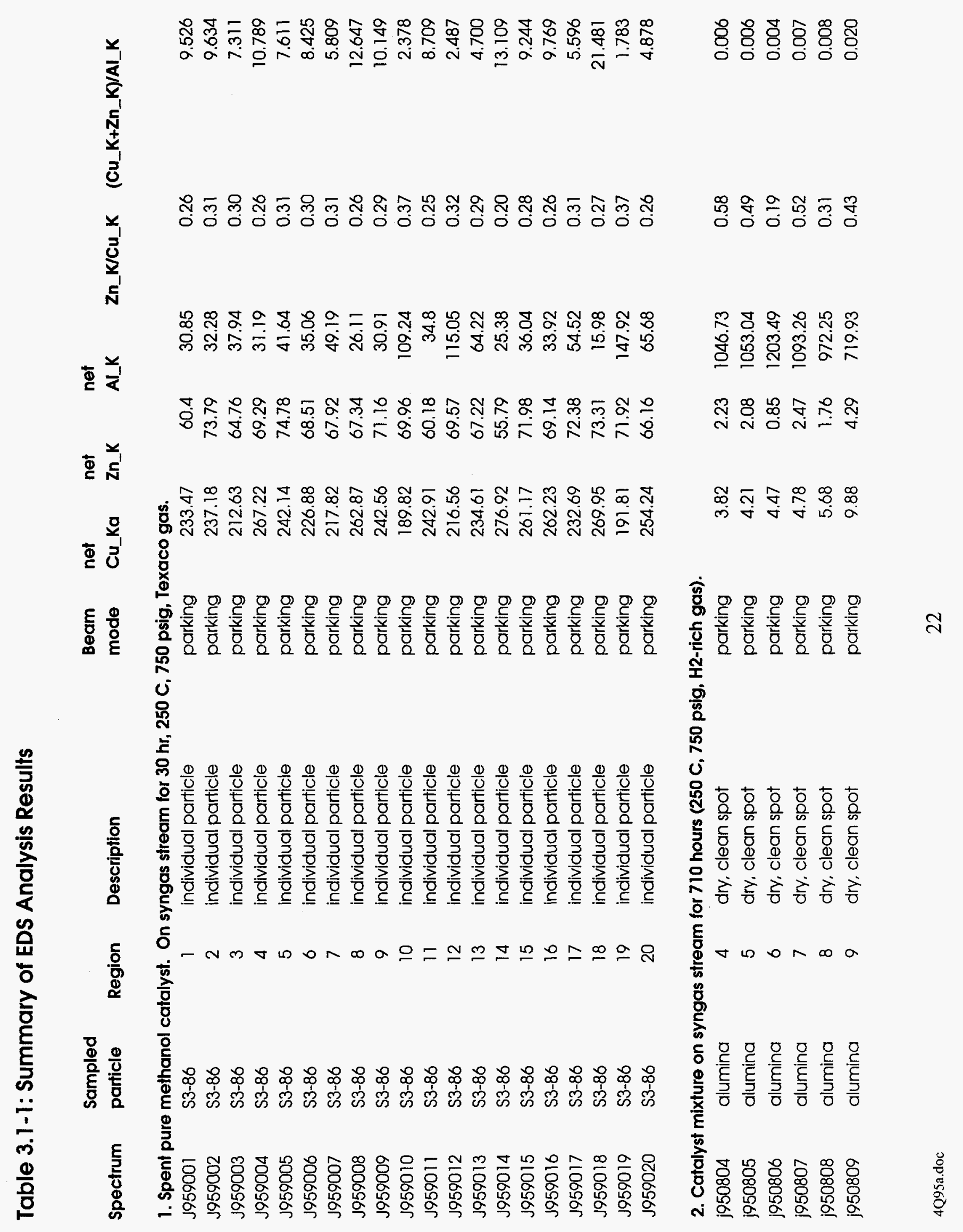




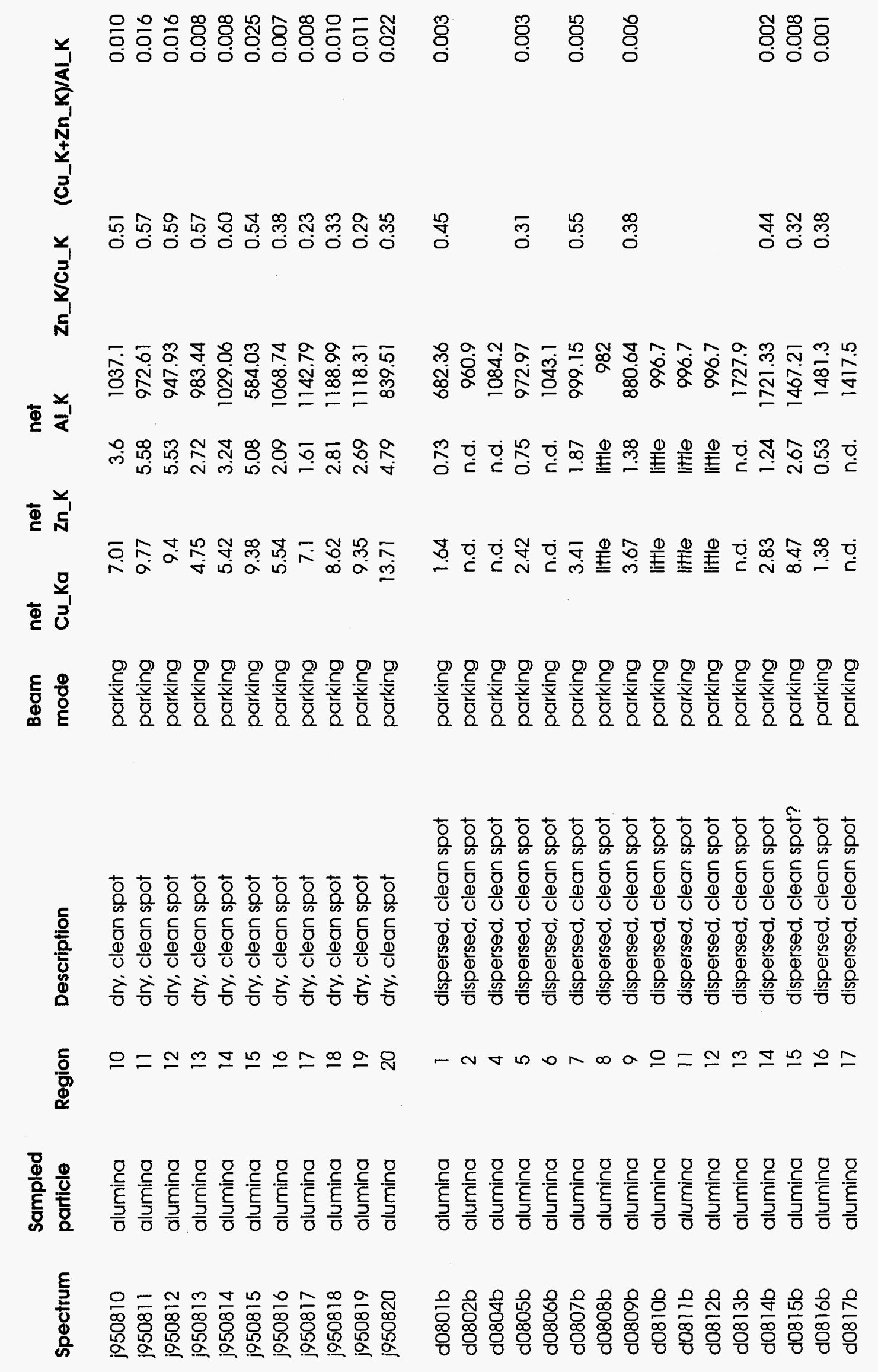




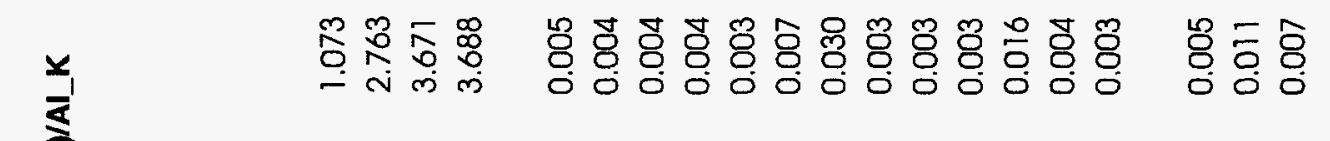

$$
\begin{aligned}
& \text { 옹 종졍 } \\
& \frac{1}{3} \\
& \text { 可废 }
\end{aligned}
$$

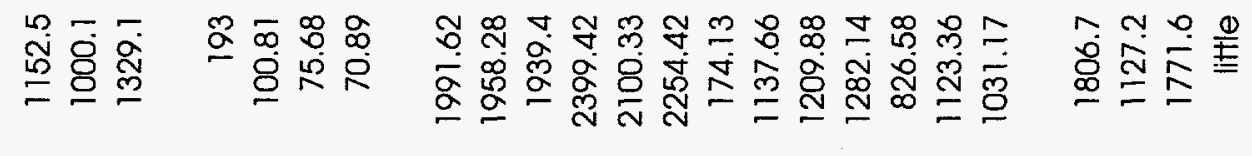

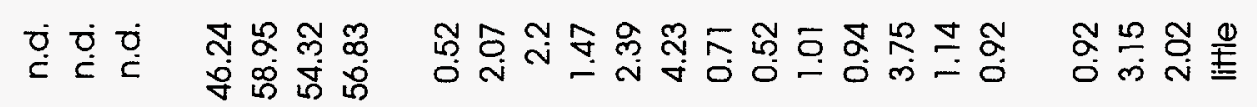

$$
\begin{aligned}
& \text { Ф节 }
\end{aligned}
$$

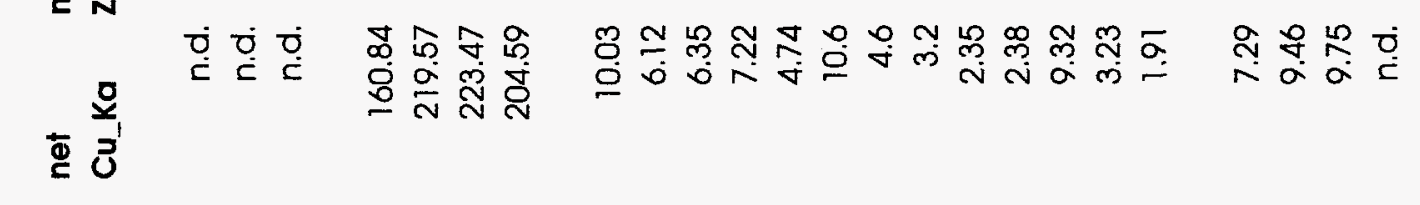

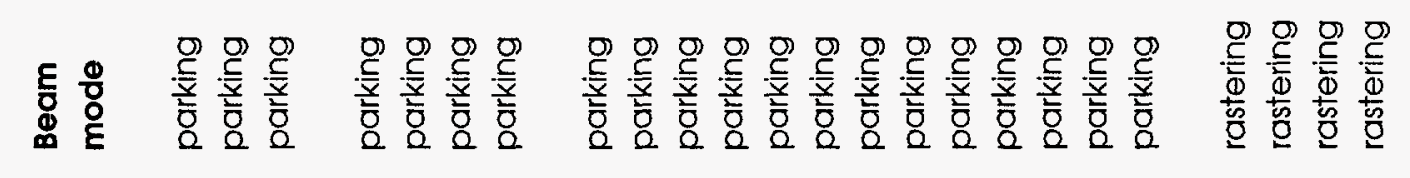

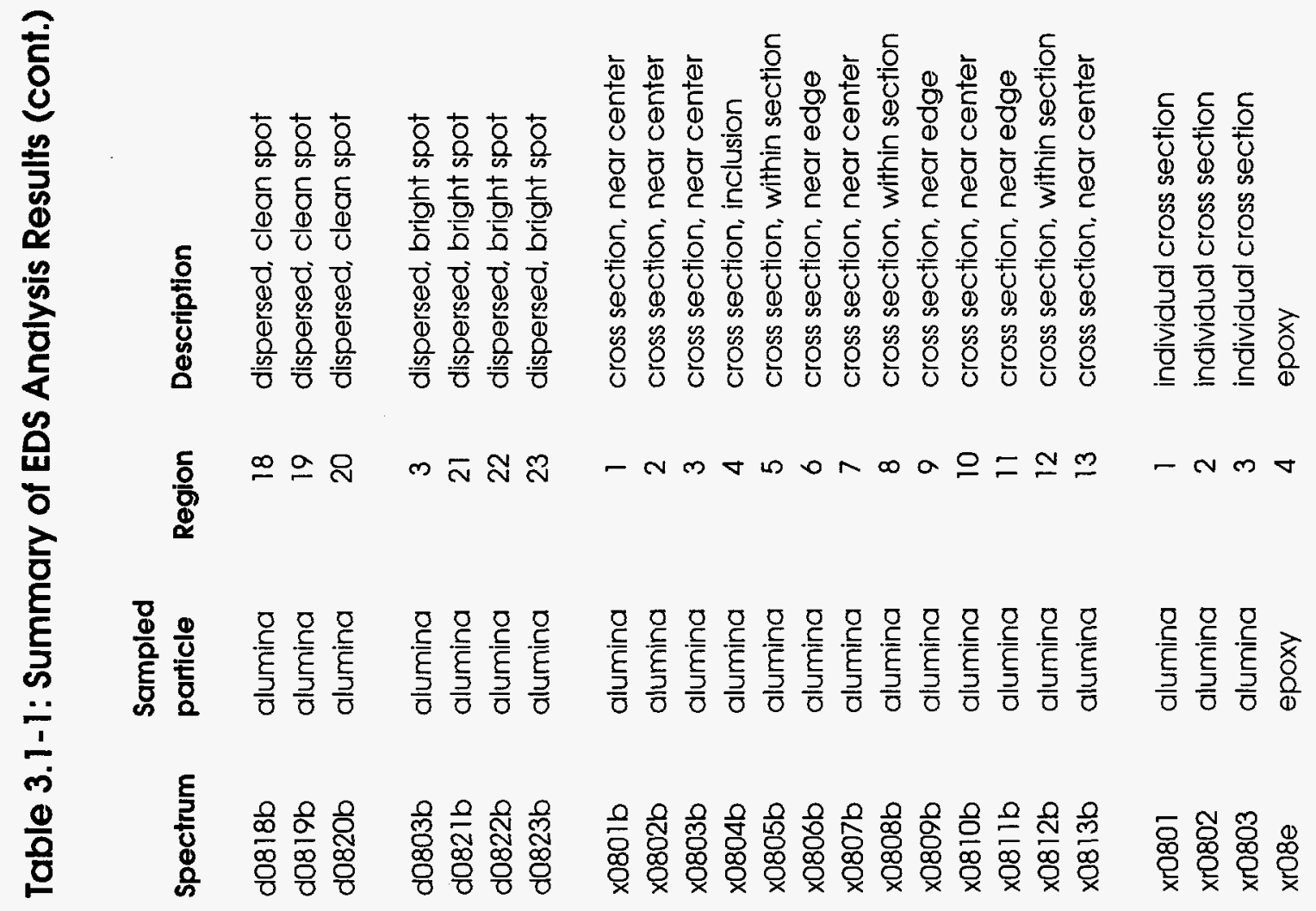




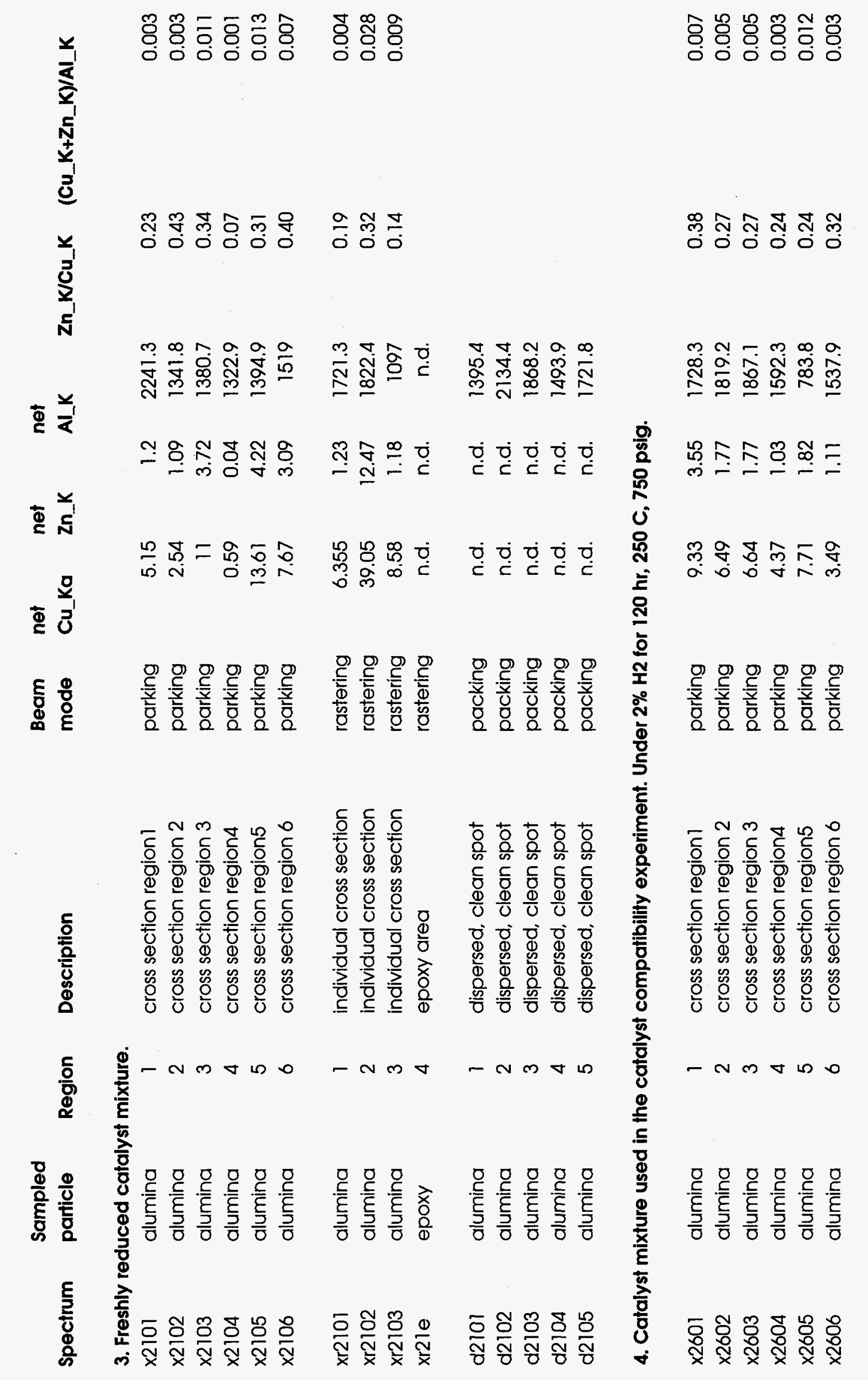




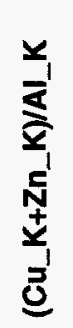

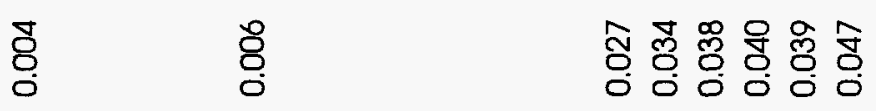

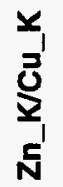

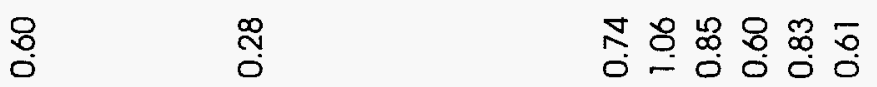

$\bar{\Phi} \frac{1}{<}$

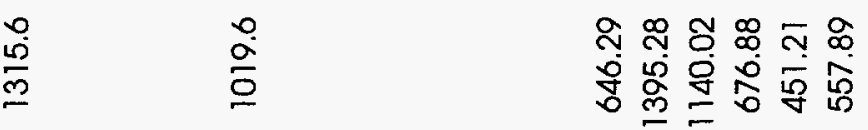

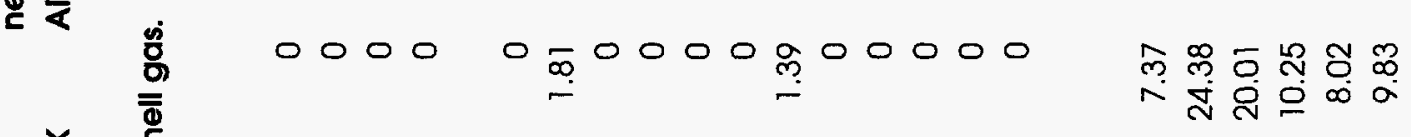

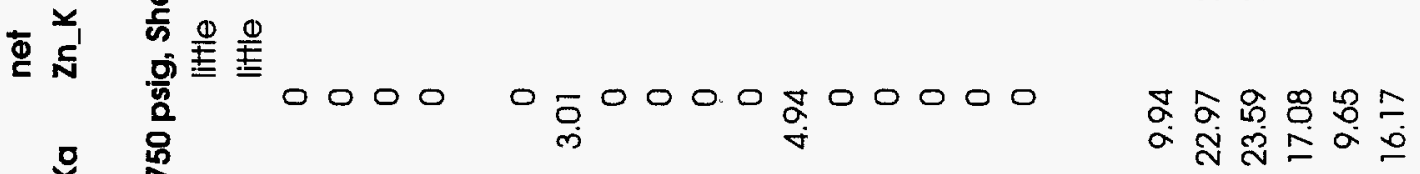

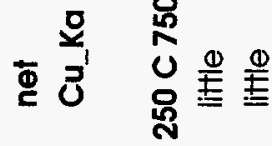

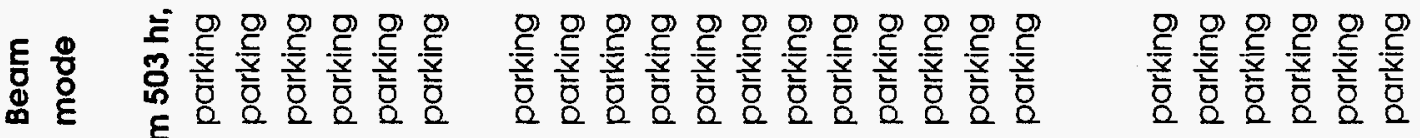

롱

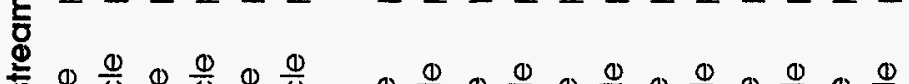

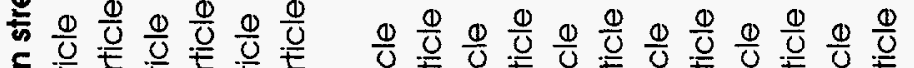

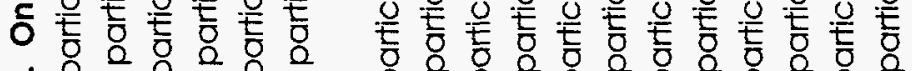

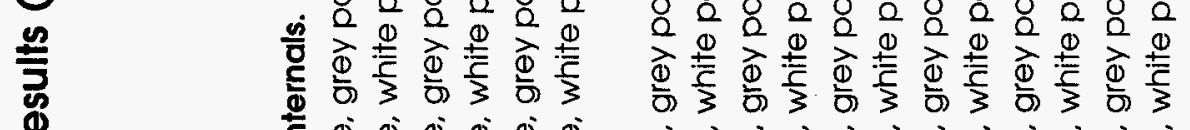

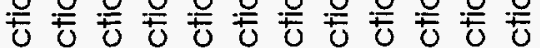

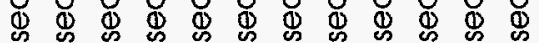

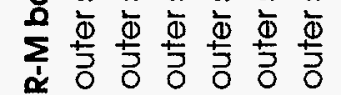

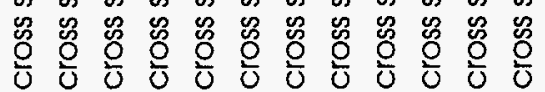

官

는

홍

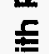
3

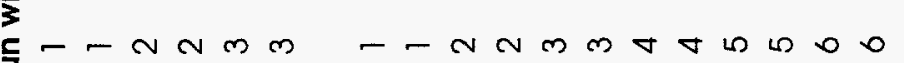

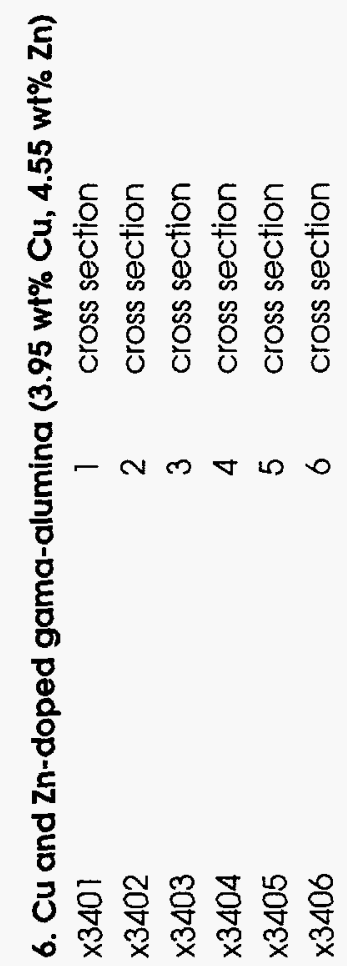



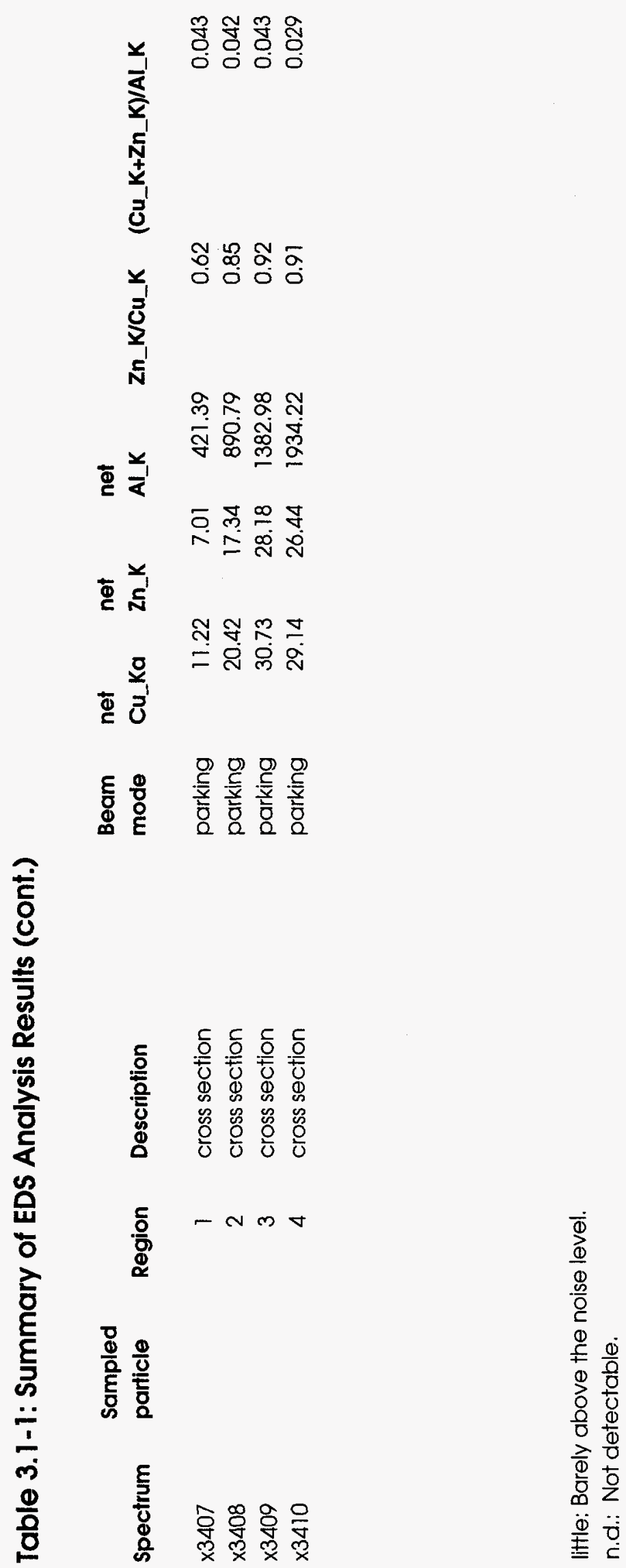
The spent sample is a catalyst mixture that was on stream for $710 \mathrm{hr}$ in a LPDME'M run. The methanol equivalent productivity dropped by $39 \%$ at the end of the run. Both the cross section and the "clean" area on the outer surface of the alumina particles in this spent mixture were analyzed with EDS. Zinc and copper were detected from all spots on the cross section and on the "clean" area of the outer surface of alumina in a dry sample. On the "clean" area of the outer surface of alumina in a dispersed sample, zinc and copper were detected on more than half of the spots (Figure 3.1.9 and Table 3.1.1).

The method used to distinguish the zinc and copper by migration from that in the methanol catalyst fines is to examine the $\mathrm{Zn}$-to-Cu ratio because it is very unlikely that zinc and copper from the migration will have the same stoichiometry as that in the methanol catalyst. The open dots in Figure 3.1.10 show the $\mathrm{Zn}$-to-Cu ratio from the spent sample. The data are very scattered compared to those from the methanol catalyst. However, they are not scattered evenly around those from the methanol catalyst, but, instead most of the data points lie above. The number average is 0.38 compared to 0.29 from the methanol catalyst. This suggests that the $\mathrm{Zn}$ and $\mathrm{Cu}$ detected from the spent sample derive from the migration of zinc- and copper-containing species during the run.

Other information supporting the migration theory is obtained from the alumina pellets used in the LPDME ${ }^{T M}$ employing Robinson-Mahoney basket internals and a mixture of catalyst pellets. The methanol catalyst did not deactivate after $508 \mathrm{hr}$ on stream in that run. The outer surface and the cross section of the alumina pellets from the run were analyzed by EDS. As shown in Table 3.1.1, $\mathrm{Zn}$ and $\mathrm{Cu}$ were not detected in most spots (16 of 18) examined. A typical EDS spectrum from this sample is shown in Figure 3.1.9f. A traditional elemental analysis using chemical digestion and atomic absorption also showed no zinc and copper buildup on the alumina. This indicates indirectly that the deactivation is related to zinc and copper migration.

Other results were puzzling. According to the migration hypothesis, the catalyst deactivation should be proportional to the extent of zinc or/and copper migration. It follows that the zinc and copper content in the freshly reduced sample (with a relative activity of 1 ) should be much lower than that in the spent sample (with a relative activity of 0.6 ). Therefore, experiments were conducted to measure the zinc and copper contents of the freshly reduced sample and compare them to that in the spent sample.

The solid dots in Figure 3.1.11 show the $(\mathrm{Zn}+\mathrm{Cu}) / \mathrm{Al}$ ratio from the cross section and "clean" area of the spent sample. The data are very scattered, ranging from 0.001 to 0.03 , with a number average of 0.008 . The three data points collected by rastering the electron beam across the crosssection of three alumina particles do not show better consistency, ranging from 0.005 to 0.01 (Table 3.1.1). No concentration gradient across alumina particles can be detected (Table 3.1.1). To estimate the concentration of zinc and copper in the alumina, a calibration sample was prepared by impregnating $\gamma$-alumina with $4.55 \mathrm{wt} \%$ zinc and $3.95 \mathrm{wt} \%$ of copper. The EDS results from this sample are shown in Figures 3.1.9, 3.1.10 and 3.1.11. The $(\mathrm{Zn}+\mathrm{Cu}) / \mathrm{Al}$ ratios from this sample, as shown in Figure 3.1.11, are also scattered, ranging from 0.027 to 0.047 with a number average of 0.038 . Based on this standard, the concentration of zinc plus copper in the spent sample ranges from 0.2 to $6.7 \mathrm{wt} \%$, with an average of $1.8 \mathrm{wt} \%$. 
Figure 3.1.11 (Cu+Zn)-to-Al EDS Ratios of Different Samples

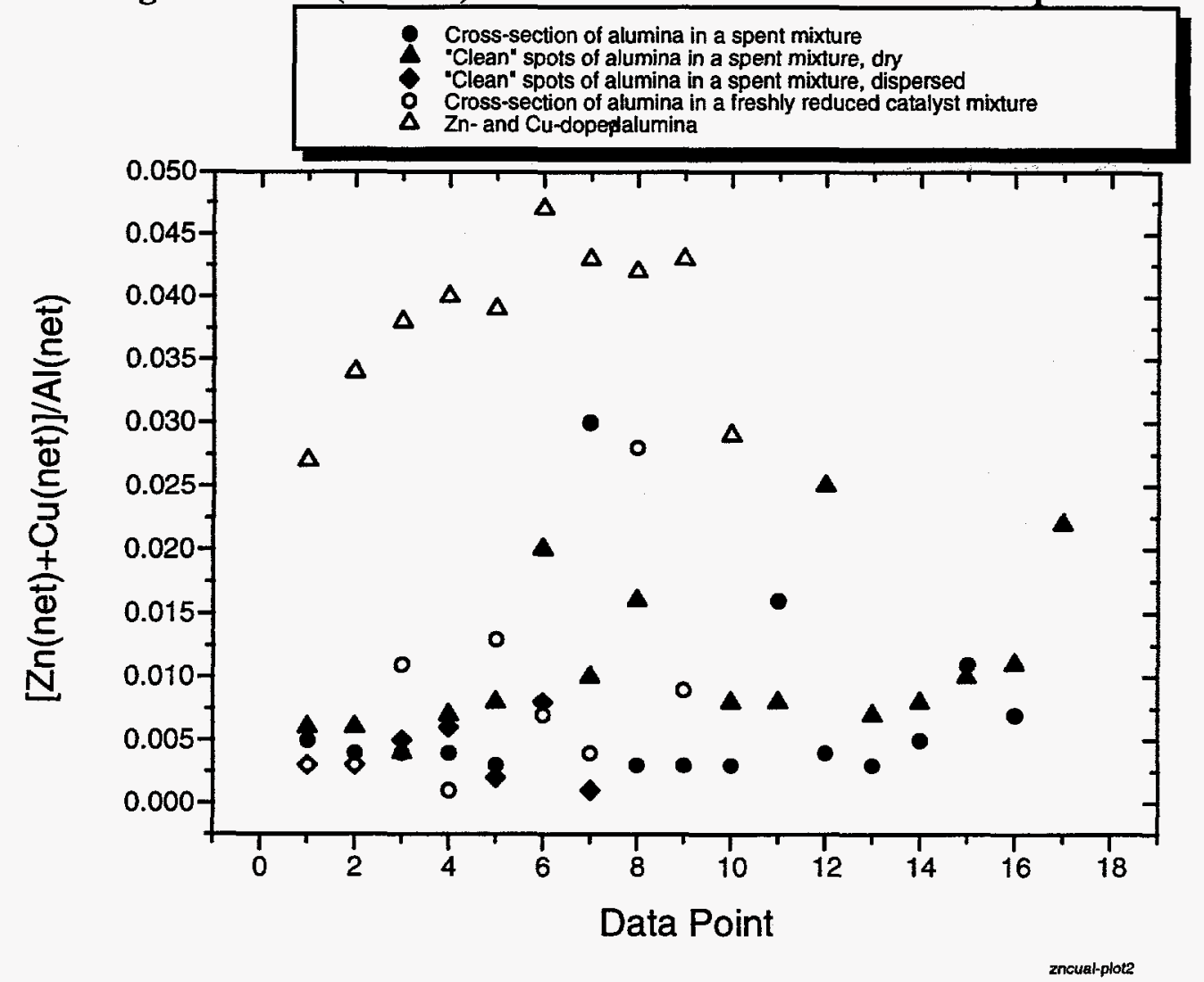

The open circles in Figure 3.1.11 are the $(\mathrm{Zn}+\mathrm{Cu}) / \mathrm{Al}$ ratios measured from the cross section of the freshly reduced sample. Zinc and copper were detected on all spots examined with EDS (Table 3.1.1). Again the data are scattered, but the range they cover is similar to that from the spent sample, with an average of 0.009 . The zinc and copper contents in these two samples are similar to each other, although their activity is different by $40 \%$. Further confusion comes from the $\mathrm{Zn}$-to- $\mathrm{Cu}$ ratio measured from the freshly reduced sample. As shown in Figure 3.1.10, the data, with an average ratio of 0.27 , are scattered around those from the methanol catalyst, with an average of 0.29 , not above them as seen with the spent sample.

Also examined was the cross section of alumina particles in the catalyst mixture used in the catalyst compatibility experiment. In this experiment, the methanol catalyst and alumina were held together under $2 \% \mathrm{H}_{2}$ in $\mathrm{N}_{2}$ and the reaction temperature $\left(250^{\circ} \mathrm{C}\right)$ and pressure (750 psig) for 120 hours. The activity of the catalyst system, in terms of methanol productivity, dropped by $50 \%$. Zinc and copper were detected from all spots examined on the cross section. As shown in Table 3.1.1, the average $(\mathrm{Zn}+\mathrm{Cu}) / \mathrm{Al}$ ratio from this sample is 0.006 . This ratio is lower than that from the spent sample (0.008), although the spent catalyst system shows lower deactivation. The $\mathrm{Zn}$-to- $\mathrm{Cu}$ ratio from the sample used in the compatibility study is 0.25 on average, again close to, but not greater than, that from the methanol catalyst (0.29).

It is certain from all of these analyses that zinc and copper are detected in the alumina particles in the catalyst mixture samples. However, it is not certain where they originate and how they are 
It is certain from all of these analyses that zinc and copper are detected in the alumina particles in the catalyst mixture samples. However, it is not certain where they originate and how they are related to the catalyst deactivation. The detected zinc and copper in the cross sections could arise from several possible causes:

1) Migration from the methanol catalyst

2) Methanol catalyst fines smeared over the cross section by the cutting

3) Methanol catalyst fines that are small enough to enter the defects and even the pores (with a mean diameter of 60 angstroms) of the alumina particles.

The first possibility is the working hypothesis. The second possibility, that of smear-over, is very unlikely because little zinc and copper were detected on the epoxy around the alumina cross sections; therefore smear-over was ignored. The third possibility is important because if it occurs, adhering methanol catalyst particles will interfere with or may overshadow analysis of the presence of any copper and/or zinc that has migrated. Thus, when the $\mathrm{Zn}$-to-Cu ratio is near that of the methanol catalyst, a definitive conclusion about migration cannot be made.

The best way to determine the third possibility is to examine the morphology of the zinc and copper inside the alumina particle (i.e., the cross section) using other techniques such as high resolution Transmission Electron Microscopy (TEM). This technique allows us to distinguish the presence of fines from atomically dispersed zinc and copper. The atomically dispersed zinc and copper arise most likely from the migrated species. If the methanol catalyst fines work their way into alumina particles, it would be impossible to verify the existence of migrated zinc and copper by elemental analysis. Nevertheless even if the third possibility were proven, it is strong evidence of intimate contact between the methanol catalyst and alumina which would allow the possibility of migration. Experiments have been designed to examine this issue using TEM.

The results discussed above demonstrate that catalyst deactivation is not correlated with $(\mathrm{Zn}+\mathrm{Cu}) / \mathrm{Al}$ ratio. Note, however, that if the zinc and copper detected derive partially or mainly from the methanol catalyst fines in the alumina, we would not expect such a correlation. If the presence of copper and zinc arises only from the migration, there could be two explanations for the lack of correlation. First, the migration may be responsible only for the initial deactivation of the catalyst system. It has been shown that deactivation during reduction contributes substantially to the initial catalyst deactivation. Therefore, once a catalyst mixture passes through the reduction stage, the zinc and copper level in the alumina will be similar. Second, the migration did occur, but it does not account for the deactivation of both catalysts. In other words, the migration hypothesis is not correct.

Finally, the quality of the EDS data was examined. As shown in Figures 3.1.10 and 3.1.11, both $\mathrm{Zn} / \mathrm{Cu}$ and $(\mathrm{Zn}+\mathrm{Cu}) / \mathrm{Al}$ ratios from the alumina samples are very scattered. Is this due to the inhomogeneous nature of the samples, or to the noise level of the EDS spectra (see Fig. 3.1.9a-e)? The inhomogeneous nature of the samples may be the more likely reason. Figures 3.1.10 and 3.1.11 show that similar scattering is observed from the $\mathrm{Zn}$ - and $\mathrm{Cu}$-doped alumina sample, while the signal-to-noise ratio in its EDS spectrum is satisfactorily low (Fig. 3.1.9). 
Then one can question if our small amount of data are statistically sound, especially for the freshly reduced and the compatibility samples.

\section{Task 3.2 New Fuels from Dimethyl Ether (DME)}

\section{Overall 4 QFY95 Objectives}

The following set of objectives appeared in Section III of the previous Quarterly Technical Progress Report No. 3:

- Continue to screen immobilized catalyst candidates for hydrocarbonylation of dimethyl ether to ethylidene diacetate.

- Continue catalyst development work on the cracking of ethylidene diacetate to vinyl acetate and acetic acid.

\section{Chemistry and Catalyst Development}

\section{(i) Dimethyl Ether to Ethylidene Diacetate (EDA)}

The effort has focused on understanding the rhodium complexes anchored to the Reillex polymers for the catalytic conversion of DME to EDA.

\section{Results and Discussion for Ionic Bound Catalyst}

It was reported previously that problems were experienced in reproducing the data from our homogeneous catalytic runs when a $100 \mathrm{cc}$ reactor instead of a $300 \mathrm{cc}$ reactor was used. Therefore, it was decided to resume using the $300 \mathrm{cc}$ reactor for further studies. The first reactions attempted were the homogeneous catalytic runs using $\mathrm{RhCl}_{3} .3 \mathrm{H}_{2} \mathrm{O}$ as the catalyst. The reaction conditions were as follows: $\mathrm{RhCl}_{3} .3 \mathrm{H}_{2} \mathrm{O}(0.2 \mathrm{~g}), \mathrm{CH}_{3} \mathrm{COOH}(145 \mathrm{~g}), \mathrm{MeI}(9.5 \mathrm{~g})$, $\operatorname{DME}(10.3 \mathrm{~g}), \mathrm{CO} / \mathrm{H}_{2}(1: 1), 1500 \mathrm{psi}, 190^{\circ} \mathrm{C}$. The overall time of reaction was $150 \mathrm{~min}$, and samples were taken at $15,30,60,90,120$, and $150 \mathrm{~min}$ for analysis by gas chromatography. The reactions were performed three separate times, and the data obtained were found to be reproducible. As reported in earlier experiments, the mass balances were found to progressively worsen with time. A typical reaction profile is shown below in Figure 3.2.1. The highest yield of EDA based on DME added was $\sim 17 \%$ at the 90 minute mark. Earlier work published in a European patent showed a much higher yield (at least 3 times greater) of EDA. Also, the results were obtained over shorter periods ( $45 \& 90 \mathrm{~min}$ ), and no samples were taken during the course of the reaction. It was decided to repeat the catalytic run in a batch mode for $60 \mathrm{~min}$ and analyze the products at the end of the reaction. The reaction profile for this one point is shown below in Figure 3.2.2.

The yield of EDA obtained in this reaction was much higher (44\%), and a comparison of the reaction profile shown in Figure 3.2.2 with the sampling mode at the 60 min mark (see Figure 3.2.1) shows interesting differences. In the batch mode the EDA concentration is higher than the acetic anhydride concentration or the acetaldehyde concentration, whereas in the sampling mode, the concentration profile is totally reversed. Based on these results it was decided to conduct heterogeneous catalysis runs in a batch mode and compare them to a sampling mode. 
Figure 3.2.1 Reaction Profile Sampling Mode

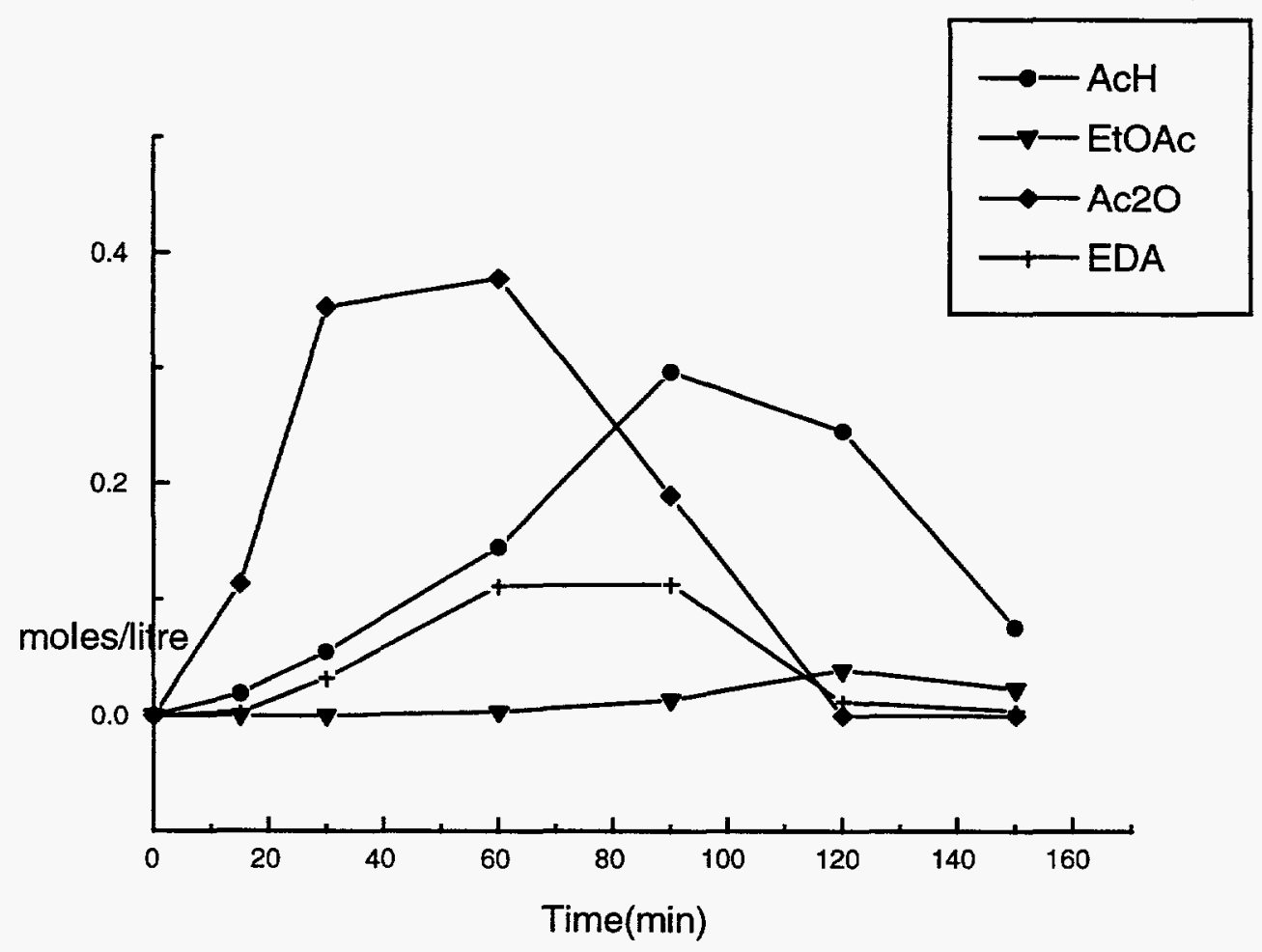

Figure 3.2.2 Reaction Profile Under Batch Mode

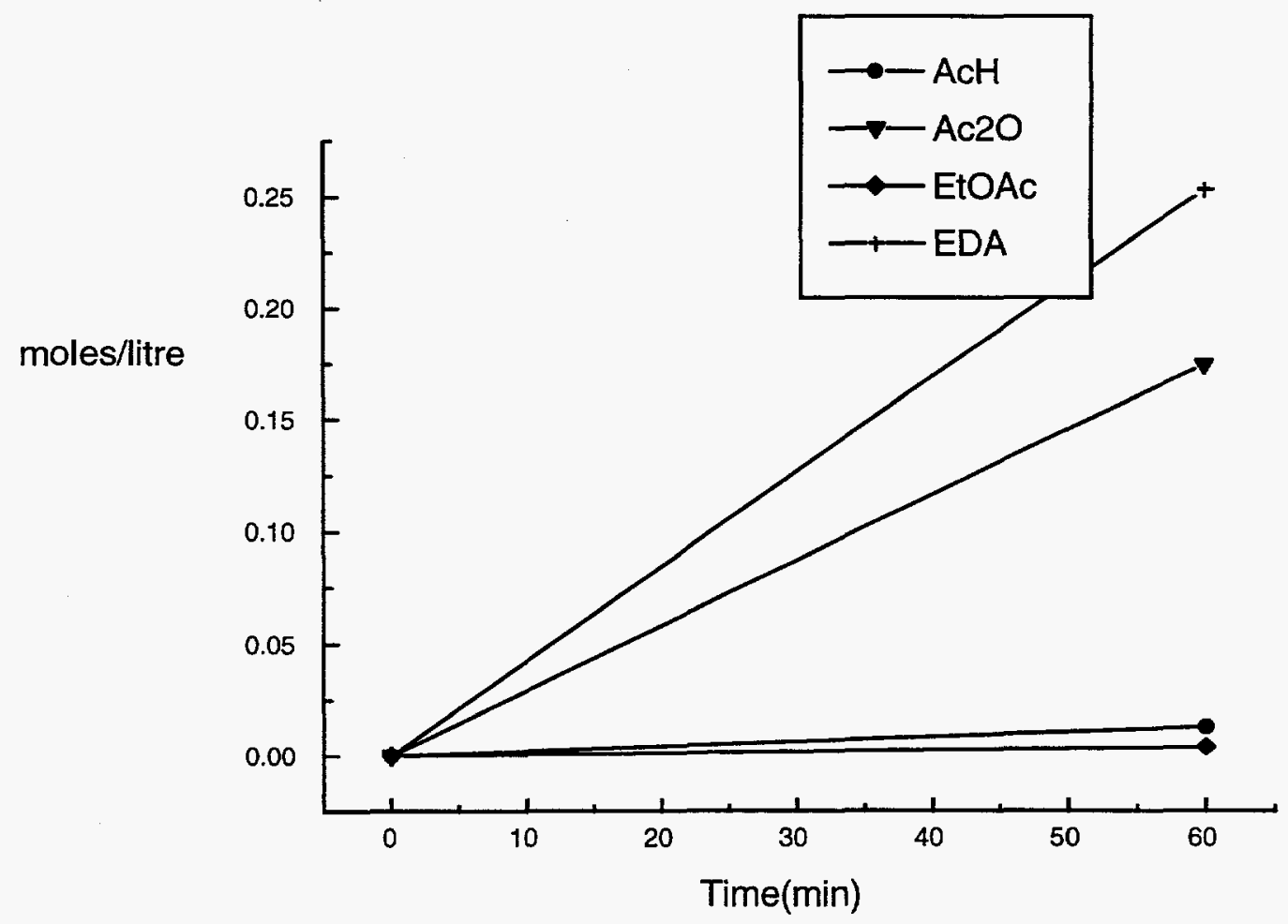




\section{Heterogeneous Catalysis (Batch vs Sampling Mode)}

The heterogeneous catalysts of interest were various Reillex polymers containing the ionically bound complex $\left[\mathrm{Rh}(\mathrm{CO})_{2} \mathrm{I}_{2}\right]^{-}$. The results of the experiments are summarized in Table 3.2.1. Also shown in this table are data from a sampling mode, Sample 1. All other data were obtained in a batch mode and include the suffix B in the sample number. The reaction conditions were similar to those described previously for the homogeneous reaction.

\section{Table 3.2.1 Results of Heterogeneous Catalytic Runs}

\begin{tabular}{ccccccccc}
$\begin{array}{c}\text { Sample } \\
\text { Number }\end{array}$ & $\begin{array}{c}\text { Time } \\
(\mathbf{m i n} .)\end{array}$ & $\begin{array}{c}\text { Conv. } \\
(\%)\end{array}$ & $\begin{array}{c}\text { MeOAc } \\
(\%)\end{array}$ & $\begin{array}{c}\text { Ac2O } \\
(\%)\end{array}$ & $\begin{array}{c}\text { AcH } \\
(\%)\end{array}$ & $\begin{array}{c}\text { EDA } \\
(\%)\end{array}$ & $\begin{array}{c}\text { EtOAc } \\
(\%)\end{array}$ & $\begin{array}{c}\text { MB } \\
(\%)\end{array}$ \\
1. & 60 & 100 & 35.3 & 30.4 & 10.5 & 12.4 & 2.2 & 90.8 \\
2. & 120 & 100 & 5.6 & 19.7 & 18.0 & 17.9 & 7.7 & 68.9 \\
3B. & 60 & 100 & 43.8 & 14.6 & 5.2 & 20.4 & 1.45 & 85.5 \\
4B. & 120 & 100 & 15.1 & 7.6 & 10.8 & 35.1 & 5.0 & 74.4 \\
5B. & 120 & 100 & 18.2 & 6.2 & 7.0 & 35.5 & 4.5 & $91^{*}$ \\
6B. & 60 & 100 & 44.7 & 10.3 & 3.8 & 22.1 & 1.8 & $93.3 *$ \\
7B. & 120 & 100 & 11.5 & 4.4 & 9.8 & 36.0 & 6.0 & $89.0^{*}$ \\
\hline
\end{tabular}

\footnotetext{
* Mass Balance (MB) includes methane. Note: $6 \mathrm{~B}, 7 \mathrm{~B}$ are REILLEX HP POLYMER; $2.24 \% \mathrm{Rh}$ 3B, 4B are REILLEX 425 POLYMER; $2.24 \%$ Rh 5B is a REILLEX 425 POLYMER; 5.1\% Rh
}

In Table 3.2.1 comparisons are made between the run labeled 3B and the run labeled 1 at the 60 minute mark. Both runs used Reillex 425 polymer containing $2.24 \%$ Rh by weight. In both of these runs the conversion of DME is close to $100 \%$; however the batch run shows a higher EDA selectivity $(20.4 \%)$ versus the sampling mode (12.4\%). Also, the batch run shows a different selectivity order $\left(\mathrm{EDA}>\mathrm{Ac}_{2} \mathrm{O}>\mathrm{AcH}\right)$ when compared to the sampling mode $\left(\mathrm{Ac}_{2} \mathrm{O}>\mathrm{EDA}>\right.$ $\mathrm{AcH})$. The batch run labeled $4 \mathrm{~B}$ and run for 120 minutes shows an EDA selectivity of $35.1 \%$, which is approximately twice that obtained for 2 at 120 minutes. Once again the product selectivity order $\mathrm{EDA}>\mathrm{AcH}>\mathrm{Ac}_{2} \mathrm{O}$ for the batch mode is different from the sampling mode: $\mathrm{Ac}_{2} \mathrm{O}>\mathrm{AcH}=\mathrm{EDA}$. The samples labeled $6 \mathrm{~B}$ and $7 \mathrm{~B}$ were obtained by using a Reillex HP polymer containing $2.24 \% \mathrm{Rh}$ by weight. Once again, Table 3.2 .1 shows that the results obtained are almost identical to the batch reactions with the Reillex 425 polymer. Finally, a batch experiment with a Reillex 425 that contained $\sim 5.1 \% \mathrm{Rh}$ by weight was run. The overall weight of material used was adjusted so as to contain the same weight of $\mathrm{Rh}$ as the earlier runs 
containing $2.24 \% \mathrm{Rh}$ by weight. The objective here was to see if the distribution of $\mathrm{Rh}$ loaded on the polymer made a difference in the catalytic activity. A comparison of 5B versus $4 \mathrm{~B}$ for 120 minutes shows almost identical results, demonstrating that it is the overall weight of rhodium that decides the activity.

\section{Conclusions}

The catalytic runs in a batch mode (homogeneous or heterogeneous) give better selectivity for EDA and a different selectivity order than the analogous reactions in a sampling mode. Future work will address understanding this effect.

\section{Results and Discussion for Covalent Bound Catalyst}

Experiments were conducted on a literature-reported heterogeneous catalyst (USP5371274) to convert MeOAc to EDA in the presence of syngas. The catalyst consisted of the complex $\mathrm{RhClCO}\left(\mathrm{PPh}_{3}\right)_{2}$ covalently linked to a phosphinated divinylbenzene polystyrene copolymer. With the described patent procedure, a polymer containing $1.8 \mathrm{wt} \% \mathrm{Rh}$ was prepared and tested as a catalyst for the MeOAc conversion to EDA of a $1: 1 \mathrm{CO} / \mathrm{H}_{2} \mathrm{mix}$ at $150^{\circ} \mathrm{C}$ and $1000 \mathrm{psi}$. The patent mentions the use of 3-picoline as an accelerator. The turnover number for EDA was calculated as moles of EDA/moles of Rh x time; a turnover number of $169 \mathrm{hr}^{-1}$ was obtained. In contrast, the same reaction without the use of promoter gave a turnover number of $15 \mathrm{hr}^{-1}$.

The reaction was also studied with the ionically bound $5.1 \% \mathrm{Rh}$ on Reillex using a comparable weight of polymer to have a weight of Rh identical to that in the covalently bound polymer. The results without promoter 3-picoline gave an EDA turnover of $14.6 \mathrm{hr}^{-1}$. In contrast, addition of 3 -picoline improved the EDA turnover to $162 \mathrm{hr}^{-1}$. Therefore the results are similar to those obtained with the covalently bound polymer. We tried to improve the turnover number without the use of 3-picoline by adding an excess of Reillex polymer to the catalytic reaction. The objective was to provide promoter sites on the heterogeneous catalyst. Although there was some improvement in EDA turnover to $\sim 20 \mathrm{hr}^{-1}$, the tremendous rate increase was not observed with 3-picoline.

Experiments were also conducted to recycle the Reillex catalyst in the presence of 3-picoline as the promoter. The yield of EDA in $4 \mathrm{hr}$ decreased dramatically from $21 \%$ in the first run to $4 \%$ in the second to $0.5 \%$ in the third run, proving that 3-picoline is leaching $\mathrm{Rh}$ from our catalyst. The novelty of the Reillex catalyst for the DME to EDA conversion is that there is no need for any promoter and the catalyst can be recycled without loss in activity. The use of the Reillex polymer also allows operation at higher temperatures $\left(190^{\circ} \mathrm{C}\right)$ compared to the gel-based polymer described in the patent.

\section{(ii) Ethylidene Diacetate to Vinyl Acetate}

\section{Background}

Ethylidene diacetate (EDA) $\left\{\mathrm{CH}_{3} \mathrm{CH}\left(\mathrm{O}_{2} \mathrm{CCH}_{3}\right)_{2}\right\}$ can be cracked to vinyl acetate (VAM) $\left\{\mathrm{CH}_{2}\right.$ $\left.=\mathrm{CHO}_{2} \mathrm{CCH}_{3}\right\}$ and acetic acid $(\mathrm{AcOH})\left\{\mathrm{CH}_{3} \mathrm{CO}_{2} \mathrm{H}\right\}$. Ethylidene diacetate (EDA) can also react to yield acetic anhydride $\left(\mathrm{Ac}_{2} \mathrm{O}\right)\left\{\left(\mathrm{CH}_{3} \mathrm{CO}\right)_{2}\right\}$ and acetaldehyde $(\mathrm{AcH})\left\{\mathrm{CH}_{3} \mathrm{C}(\mathrm{O}) \mathrm{H}\right\}$. Reaction 1 depicts this series. 


\section{$\mathrm{Ac2O}+\mathrm{AcH} \longleftrightarrow \mathrm{EDA} \longleftrightarrow \mathrm{VAM}+\mathrm{AcOH} \quad \mathrm{Rxn} .1$}

In order to suppress the reaction to $\mathrm{Ac}_{2} \mathrm{O}$, previous work involved co-feeding $\mathrm{Ac}_{2} \mathrm{O}$. Previous cracking work also involved an acid catalyst such as p-TSA (para-Toluene Sulfonic Acid).

\section{Gas Phase Cracking}

Several catalysts, including an acidic carbon, a de-aluminized $\mathrm{Y}$ zeolite, and several coated carbons, were examined in a standard gas phase flow system. One gram samples of catalysts were loaded in a stainless steel reactor tube and brought to a temperature of $180^{\circ} \mathrm{C}$ in flowing nitrogen $(10 \mathrm{cc} / \mathrm{min})$. A feed of ethylidene diacetate, $0.2 \mathrm{ml} / \mathrm{hr}$ liq., was vaporized and introduced and the reaction products were monitored by gas chromatography. GC confirmed the presence of acetic anhydride, acetic acid, acetaldehyde, and unreacted ethylidene acetate. Based upon the acetic acid produced, the VAM production should have been substantial. The absence of vinyl acetate was unexplained.

Based on the quantity of acetic acid produced, the absence of VAM was disturbing. A series of temperature profile runs with VAM as a feedstock confirmed that vinyl acetate was thermally stable at temperatures up to $180^{\circ} \mathrm{C}$ in our reactor system. Further testing with a VAM feedstock and a catalyst in place confirmed that the catalyst was neither destroying nor adsorbing the VAM. The absence of acetylene in the product stream also indicated that the VAM was not being decomposed in another pathway.

In order to suppress the reverse reaction to acetic anhydride and acetaldehyde, the liquid feed was changed to a 50:50 mole \% $\mathrm{EDA} / \mathrm{Ac}_{2} \mathrm{O}$ feed. Other parameters were maintained as they were previously. The product slate was essentially the same as seen above: $\mathrm{Ac}_{2} \mathrm{O}, \mathrm{AcOH}, \mathrm{AcH}$, and EDA. The presence of acetaldehyde indicated that EDA was still being reacted to $\mathrm{Ac}_{2} \mathrm{O}$ and $\mathrm{AcH}$, even though $\mathrm{Ac}_{2} \mathrm{O}$ was a substantial component of the feed.

The most plausible explanation for this unexpected chemistry involves the probable hydroxyl sites present on the catalyst surface. If these sites react with $\mathrm{Ac}_{2} \mathrm{O}$, they yield acetic acid and leave acetate on the catalyst. In this reaction, the $\mathrm{Ac}_{2} \mathrm{O}$ concentration is lowered, allowing EDA to react to produce more $\mathrm{Ac}_{2} \mathrm{O}$ and $\mathrm{AcH}$, thus explaining the presence of $\mathrm{AcH}$ and the absence of VAM. Reactions 2 and 3 diagram this.

$$
\begin{aligned}
& \mathrm{Ac} 2 \mathrm{O}+\mathrm{HO}-\longrightarrow \mathrm{AcOH}+\mathrm{AcO}-\quad \mathrm{Rxn} 2 \\
& \mathrm{EDA} \longrightarrow \mathrm{Ac2O}+\mathrm{AcH} \quad \mathrm{Rxn3}
\end{aligned}
$$

\section{Liquid Phase Cracking}

Attempts were initiated to examine cracking of EDA in the liquid phase. A 15 gram sample of EDA was loaded into a $50 \mathrm{cc}$ stirred reactor, and a 0.2 gram sample of ion-exchange resin (IER) was added. The reactor was sealed and heated to $150^{\circ} \mathrm{C}$ with stirring. After 4 hours, the reactor contents were transferred through a filter into an evacuated sample bomb. Subsequent analysis by manual injection showed that no reaction had taken place. At $150^{\circ} \mathrm{C}$, with a known strong acid catalyst, no reaction occurs in a closed system. Based upon this, several conclusions can be drawn concerning the equilibrium of reaction 1 . 
K1 must be large with respect to formation of EDA. K2 must be small and hence formation of VAM is unfavored.

\section{Distillation Cracking}

Based upon the equilibrium deductions above, in order to drive the reaction toward VAM and $\mathrm{AcOH}$, one or both of the products must be removed as it is produced. A simple distillation with reflux was constructed. EDA and an IER (ion exchange resin) were loaded into a round bottom flask and brought to boiling. The distillate collected showed acetaldehyde, acetic anhydride, acetone, and vinyl acetate. Analysis of the pot showed DME (or a compound with the same retention time), acetic acid, acetic anhydride, and unreacted EDA. This product slate matches the predicted chemistry. More important, the presence of significant amounts of VAM is encouraging. Mass balances and conversions will be reported next quarterly.

An attempt to crack EDA thermally, without catalyst, led to simply distilling the EDA with no cracking. An attempt to base catalyze the reaction using $\mathrm{MgO}$ also led to no reaction.

Future work will examine a continuous distillation method to crack EDA to VAM while eliminating the reaction to $\mathrm{Ac}_{2} \mathrm{O}$. The goal is to reduce the cracking temperature to $100-110^{\circ} \mathrm{C}$, which is within the thermal limits of an IER.

\section{Q FY96 Objectives}

Future plans for Task 3.2 will focus on the following areas:

- Continue to screen immobilized catalyst candidates for hydrocarbonylation of dimethyl ether to ethylidene diacetate.

- Continue catalyst development work on the cracking of ethylidene diacetate to vinyl acetate and acetic acid.

\section{Value Added Acetyls From Syngas (Eastman Chemical Company)}

\section{A. Introduction}

The overall objective of this project is to produce a commercially viable process for the generation of vinyl acetate monomer (VAM) based entirely upon coal generated syngas (Scheme 1). Previous attempts at this objective have generally involved the combination of acetic anhydride (generated by carbonylation of either dimethyl ether or methyl acetate) with acetaldehyde (generated by either hydrogenation of acetic anhydride $\left(\mathrm{Ac}_{2} \mathrm{O}\right)$ or hydrocarbonylation of either methanol $(\mathrm{MeOH})$ or a methyl ester) to generate ethylidene diacetate (EDA), which is subsequently cracked to form VAM in a separate step. An exemplary process is shown below:

$$
2 \mathrm{CO}+4 \mathrm{H}_{2} \quad \rightarrow \quad 2 \mathrm{MeOH}
$$




$\begin{array}{lll}2 \mathrm{MeOH}+2 \mathrm{AcOH} & \rightarrow & 2 \mathrm{AcOMe}+2 \mathrm{H}_{2} \mathrm{O} \\ \mathrm{AcOMe}+\mathrm{CO}+\mathrm{H}_{2} & \rightarrow & \mathrm{AcH}+\mathrm{AcOH} \\ \mathrm{AcOMe}+\mathrm{CO} & \rightarrow & \mathrm{Ac}_{2} \mathrm{O} \\ \mathrm{Ac}_{2} \mathrm{O}+\mathrm{AcH} & \rightarrow & \mathrm{EDA} \\ \mathrm{EDA} & \rightarrow & \mathrm{VAM}+\mathrm{AcOH}\end{array}$

$\mathrm{AcH}=$ acetaldehyde

$\mathrm{AcOMe}=$ methyl acetate

These efforts have failed to generate a commercially viable process to date. One of the key reasons for this failure was the very large quantities of recycled acetic acid (and consequently large commercial facilities) inherent in the earlier proposed processes.

Eastman's proposal was to circumvent the recycle problem by generating AcH by hydrogenation of acetic acid $(\mathrm{AcOH})$ instead of by reductive carbonylation. Unfortunately, this process is thermodynamically disfavored and, even if acetic acid is hydrogenated, the conditions required generally favor further hydrogenation to form ethanol and ethyl acetate, which are the thermodynamically favored products. Currently, any processes that have successfully hydrogenated a carboxylic acid circumvent this problem by operating at unacceptably high pressures and temperatures to overcome the thermodynamic constrictions and by operating at low conversion to minimize over hydrogenation to the alcohol.

Eastman's proposed solution to this dilemma was to convert the acetic acid to ketene (a very well known process) and utilize the high energy content of the unstable ketene intermediate to overcome the thermodynamic constrictions to hydrogenation. The key task would be to identify catalysts that hydrogenated the ketene intermediate selectively to acetaldehyde (particularly did not generate ethanol or ethyl acetate) and would do so at commercially desirable temperatures and pressures.

Several restrictions are inherent in the contemplated conversion. Due to the unstable nature of ketene, the vapor pressure of ketene in the process should be less than atmospheric and its conversion should be reasonably high. Further, recovery of acetaldehyde will require that there not be excessive amounts of additional hydrogen present. Prior to this study, no catalyst was known for accomplishing this task.

Whereas the hydrogenation of acetic acid represented the linchpin technology in the proposal, the proposal also included some advances in the subsequent conversion of acetaldehyde to VAM. Obviously, the acetaldehyde thus formed could be converted to EDA and subsequently to VAM by known methods; Eastman proposed several improvements upon this known process. However, Eastman also proposed a very speculative application of ketene for the direct esterification of acetaldehyde to yield VAM without the intermediate generation of significant amounts of EDA. If this speculative conversion came to fruition, the overall process would be represented by the following relatively simple scheme: 


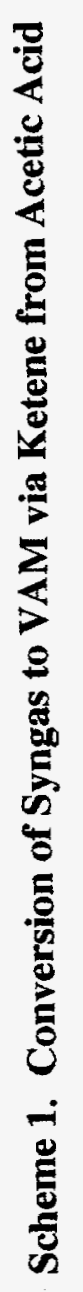

目

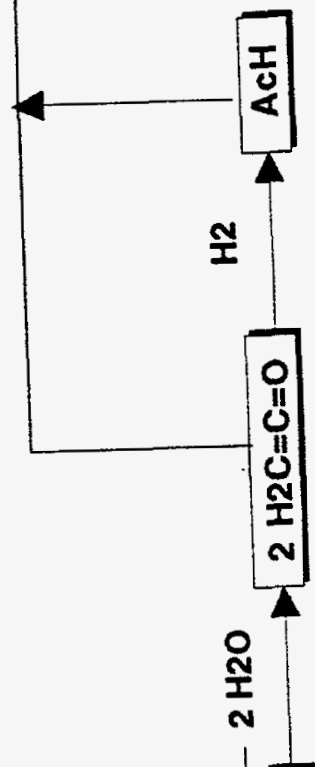

ำ

$\infty$

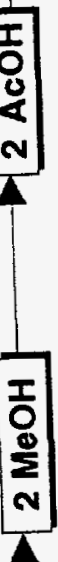

$*$
$⿱ ㇒$
$\sigma$
+
0
0
$N$ 
It is particularly important to point out that this scheme does not have any significant recycle loops. Further, due to the nature of the process, there are numerous opportunities to reduce capital costs through proper integration, and there is a notable economy of scale associated with the size of the intermediate steps.

Consistent with these goals, Eastman has pursued the following tasks in the 4th quarter of 1995: Task 1.1. Examine hydrogenation of $\mathrm{AcOH}$ to acetaldehyde via ketene.

Task 2.1. Examine the direct conversion of $\mathrm{AcH}$ and ketene to VAM.

Task 3.1.a. Assess preliminary economics for a process based on ketene hydrogenation.

\section{B. Results and Discussion}

Task 1.1 Hydrogenation of AcOH via Ketene Intermediates. Over the last several months, Eastman has explored several combinations of different reactor designs for bench-scale production, handling, and hydrogenation of ketene at varying production levels. Ketene can be generated readily from any of four sources, namely, diketene, acetic anhydride, acetic acid, and acetone. Although the preferred method of generating ketene on the commercial scale is via the cracking of acetic acid, a process practiced commercially for more than 70 years, researchers at Eastman are currently using acetone as the source of ketene since the acetone-based processes are easier to operate at the bench scale.

The general method for Eastman's initial studies of the hydrogenation of ketene is represented in the simplified depictions in Schemes 2 and 3; details on the internals, several safety outlets, traps, and a pressure equalizing system for clarity have been omitted. (Scheme 2 represents the generation of ketene from acetone, but a similar apparatus was used for the generation of ketene from acetic anhydride in the earliest work.)

For its initial work, Eastman generated ketene in a separate reactor and stored it temporarily in a dry ice trap. The ketene was then introduced as a gas stream of nitrogen saturated with ketene at $-78^{\circ} \mathrm{C}$. This produced a gas stream with a ketene partial pressure of about 150 torr (i.e., about $20 \%$ ketene in nitrogen). The rate of ketene introduction was first calibrated using a methanol scrubber that quantitatively converts ketene to methyl acetate. The stream was then diverted to the hydrogenation reactor. At the head of the reactor, the ketene stream was mixed with hydrogen (hydrogen/ketene $=$ ca. 2 ) and introduced into a heterogeneous catalyst bed maintained at steam temperature $\left(100^{\circ} \mathrm{C}\right)$ and 1 atmosphere total pressure. The effluent was passed through the same type of analytical scrubber used for calibration, except it was fitted with a dry ice condenser to capture acetaldehyde. Unreacted ketene was again converted to methyl acetate. 
है

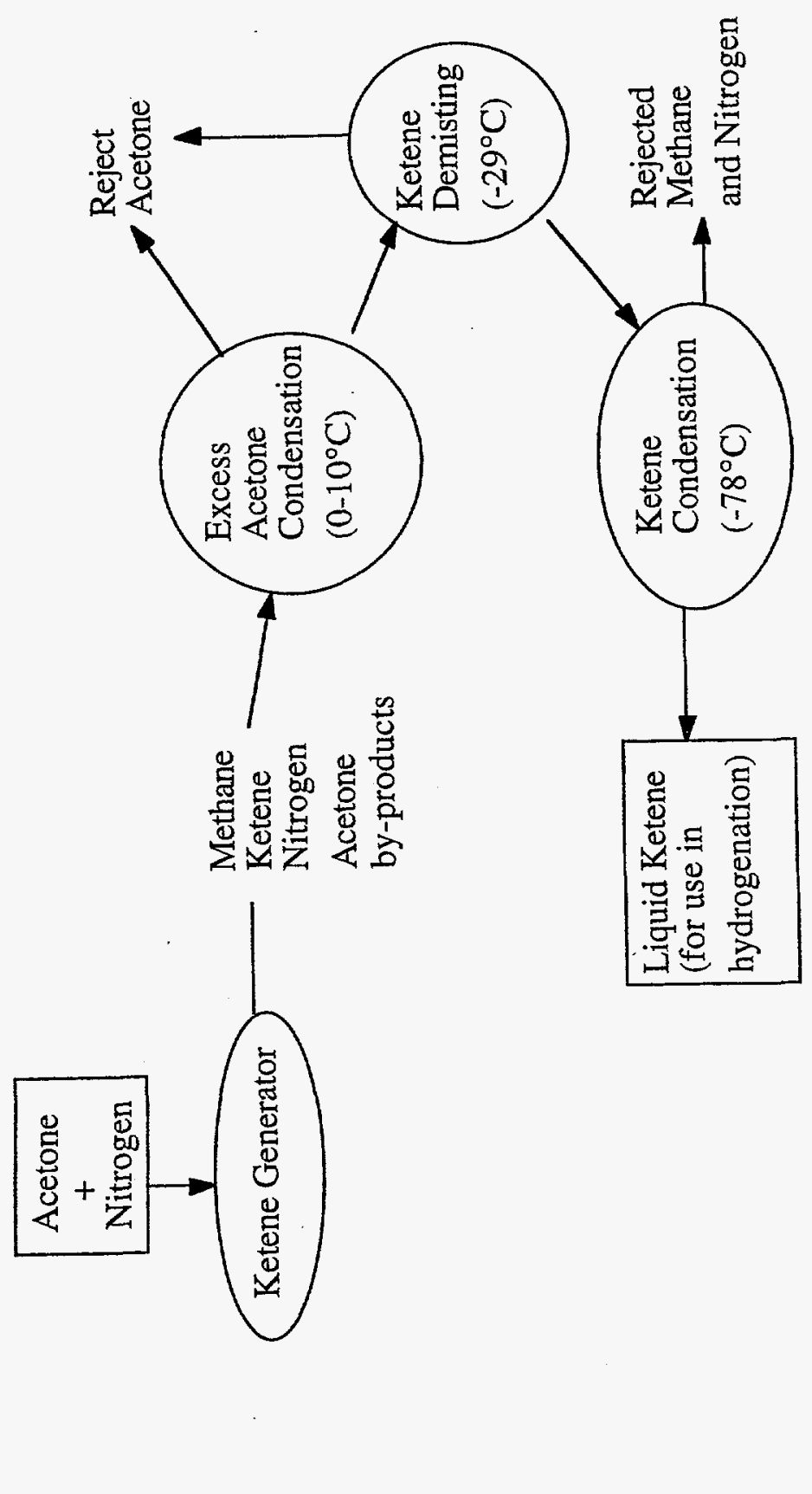


Scheme 3. Description of the Hydrogenation Apparatus

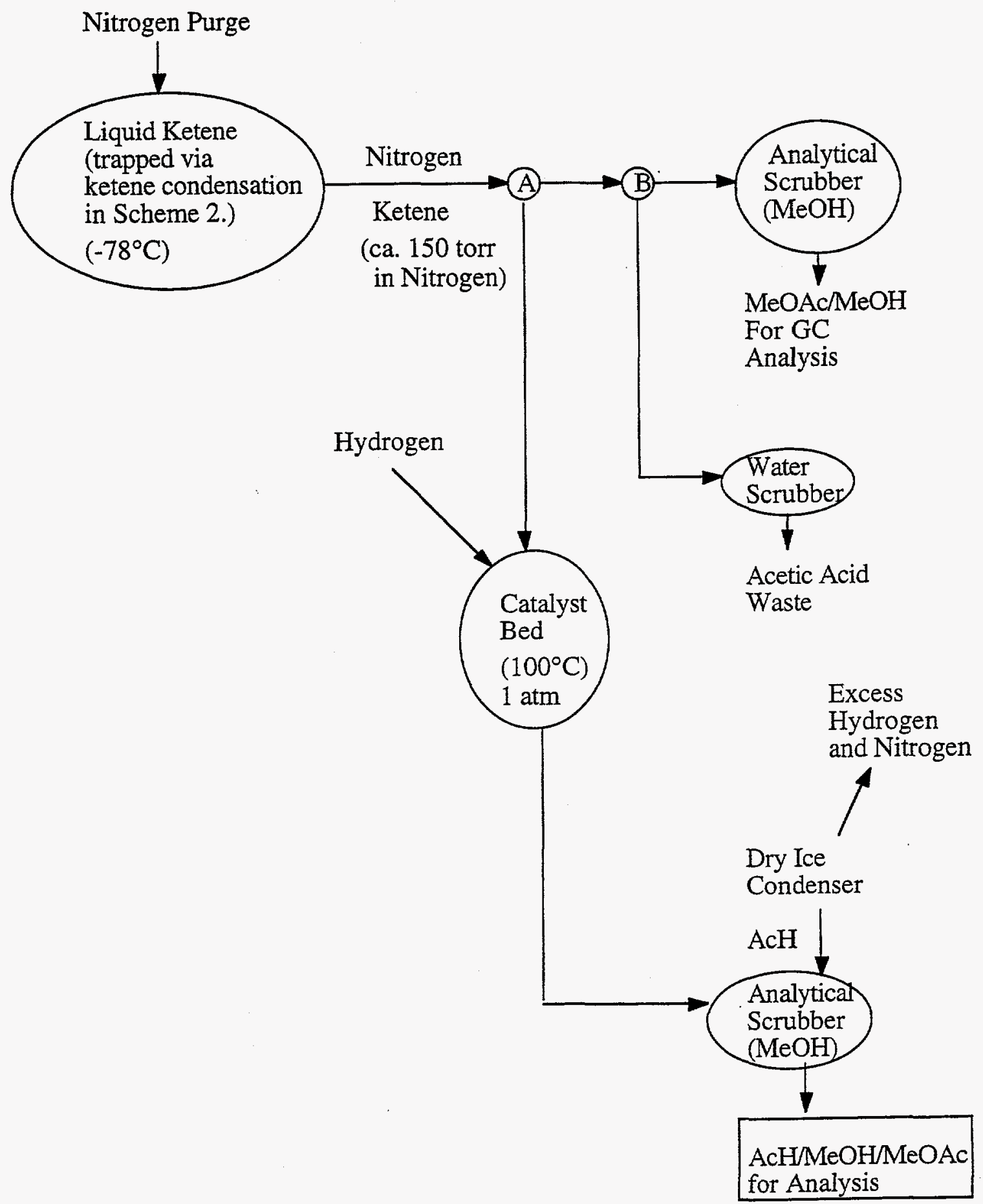


Eastman has examined a number of heterogeneous catalysts using this method. To date, the only catalysts that have shown activity under these conditions are based on $\mathrm{Pd}, \mathrm{Pt}$, and $\mathrm{Rh}$. However, $\mathrm{Pd}$ has shown much higher rates and selectivities than Pt or Rh, and Eastman has conducted several studies to extend the types of Pd-based catalysts that might prove useful. Results of this study are summarized in Table 3.2.2. In addition, Eastman has conducted a brief, qualitative survey of the effect of time on stream for several of the catalysts (Table 3.2.3) along with a more detailed study of the durability of a Pd sponge catalyst (Figures 3.2.3-3.2.6.) The results to date are excellent for a bench-scale unit. This represents the first direct hydrogenation of ketene to our knowledge, although it has been invoked previously as a likely, but unproven, intermediate in the hydrogenation of acetic acid.

Task 2.1 Direct Conversion of Ketene and Acetaldehyde to VAM. Although methods existed for the conversion of acetaldehyde and syngas-derived acetic anhydride to VAM, Eastman perceived a potential advantage to using the ketene directly for the conversion of acetaldehyde to vinyl acetate. Traditionally, the conversion was carried out by mixing acetic anhydride and acetaldehyde and isolating the vinyl acetate from the equilibrium mixture (represented by the two equations shown below), even though the equilibria favored EDA formation.

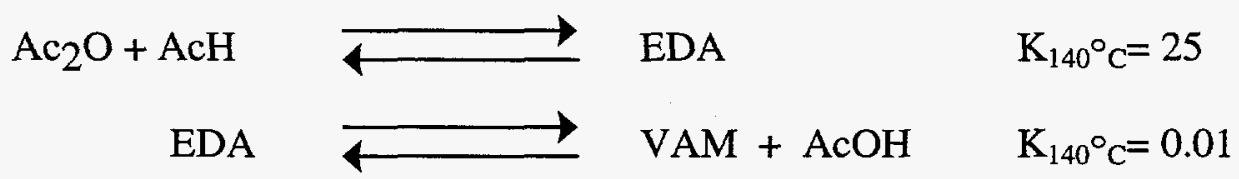

Eastman envisioned this addition as a means to consume the $\mathrm{AcOH}$ in situ by reaction of ketene as follows:

$$
\mathrm{H}_{2} \mathrm{C}=\mathrm{C}=\mathrm{O}+\mathrm{AcOH} \longrightarrow \quad \mathrm{Ac}_{2} \mathrm{O}
$$

The operating temperatures of this reaction $\left(<140^{\circ} \mathrm{C}\right)$ would render it essentially irreversible, and the process should be driven toward the generation of previously disfavored vinyl acetate by the continuous consumption of acetic acid. This process not only has the potential advantage of improving the efficiency of VAM formation, but would replace the acetic anhydride generation facility in earlier concepts with a simplified acetic acid process. (Purity requirements for ketene generation are much less strict than for other acetic acid applications.) 
Table 3.2.2 Experimental Results for the Hydrogenation of Ketene to Acetaldehyde

\begin{tabular}{|c|c|c|c|c|c|c|c|c|}
\hline Gatallist: & 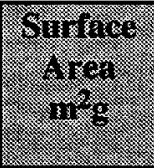 & 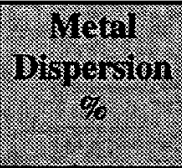 & 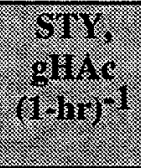 & 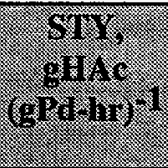 & 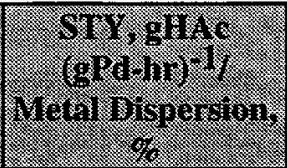 & 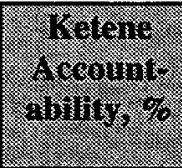 & 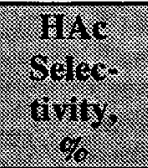 & 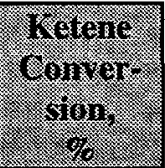 \\
\hline $\begin{array}{c}0.5 \% \mathrm{Pd} / \mathrm{Al}_{2} \mathrm{O}_{3} \\
\text { pellets }\end{array}$ & 238 & 19.5 & 188 & 44.7 & 2.29 & 84 & 72 & 59 \\
\hline $5 \% \mathrm{Pd} / \mathrm{BaSO}_{4}$ & 4.2 & 2.6 & 1416 & 25.4 & 9.77 & 70 & 70 & 78 \\
\hline $\begin{array}{c}5 \% \mathrm{Pd} / \mathrm{CaCO} 3 \\
w / \mathrm{Pb}\end{array}$ & 6.42 & 2.9 & 736 & 17.6 & 6.07 & 56 & 43 & 77 \\
\hline $5 \% \mathrm{Pd} / \mathrm{CaCO}_{3}$ & 13.99 & 10.7 & 1151 & 32.1 & 3 & 79 & 74 & 82 \\
\hline $5 \% \mathrm{Pd} / \mathrm{BaCO}_{3}$ & 5.21 & 2.9 & 1392 & 25.1 & 8.66 & 81 & 72 & 67 \\
\hline $5 \% \mathrm{Pd} / \mathrm{C}$ & 1259 & 25.1 & 713 & 37.1 & 1.48 & 78 & 76 & 92 \\
\hline $\begin{array}{c}0.5 \% \mathrm{Pd} / \mathrm{Al}_{2} \mathrm{O}_{3} \\
\text { powder }\end{array}$ & 103 & 17.1 & 1639 & $\overline{31.3}$ & 1.83 & 82 & 77 & 77 \\
\hline $1 \% \mathrm{Pd} / \mathrm{TiO}_{2}$ & 9 & 1.7 & 108 & 15.8 & 9.29 & 88 & 68 & 36 \\
\hline $3 \% \mathrm{Pd} / \mathrm{SiO}_{2}$ & 55 & 14.4 & 400 & 33.3 & 2.31 & 83 & 79 & 80 \\
\hline$* 5 \% \mathrm{Pd} / \mathrm{BaCO}_{3}$ & 5.21 & 2.9 & 559 & 9.3 & 3.21 & 83 & 54 & 37 \\
\hline Pd Sponge & 0.13 & 0.004 & 1180 & 0.47 & 117.5 & 91 & 66 & 27 \\
\hline
\end{tabular}

Data presented is from the performance of the fourth sample.

All reactions were run in a fixed catalyst bed, steam-heated reactor except for *'ed $\mathrm{Pd} / \mathrm{Ba} 2 \mathrm{CO} 3$ run which was run in slurry phase reactor.

Table 3.2.3 Percent Change in Performance Between 4th and 16th Sample

\begin{tabular}{|c|c|c|c|c|c|}
\hline $\begin{array}{l}\text { Ref } 190 \\
224858\end{array}$ & Catalyst: & Rate & 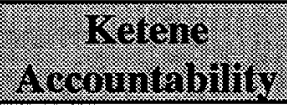 & 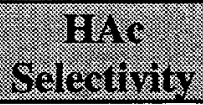 & 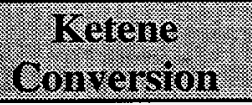 \\
\hline 014 & $0.5 \% \mathrm{Pd} / \mathrm{Al}_{2} \mathrm{O}_{3}$ pellets & -32 & -2 & -14 & -22 \\
\hline 033 & $5 \% \mathrm{Pd} / \mathrm{BaSO}_{4}$ & -3 & +7 & -7 & -8 \\
\hline 038 & $5 \% \mathrm{Pd} / \mathrm{CaCO}_{3} \mathrm{w} / \mathrm{Pb}$ & -18 & +16 & +2 & -19 \\
\hline 043 & $5 \% \mathrm{Pd} / \mathrm{CaCO}_{3}$ & -6 & -28 & -23 & +23 \\
\hline 048 & $5 \% \mathrm{Pd} / \mathrm{BaCO}_{3}$ & -11 & +6 & +6 & -16 \\
\hline 085 & $5 \% \mathrm{Pd} / \mathrm{C}$ & -16 & +14 & +11 & -24 \\
\hline 060 & $0.5 \% \mathrm{Pd} / \mathrm{Al}_{2} \mathrm{O}_{3}$ powder & -41 & -33 & -43 & +4 \\
\hline 066 & $1 \% \mathrm{Pd} / \mathrm{TiO}_{2}$ & +1 & -14 & -25 & +33 \\
\hline 072 & $3 \% \mathrm{Pd} / \mathrm{SiO}_{2}$ & -14 & +10 & +8 & -20 \\
\hline 058 & $5 \% \mathrm{Pd} / \mathrm{BaCO}_{3}$ & \multicolumn{4}{|c|}{ slurry phase } \\
\hline 077 & Pd Sponge & +0.4 & +1 & +6 & -4 \\
\hline
\end{tabular}


Figure 3.2.3 HAc STY vs Time On Stream Pd Sponge

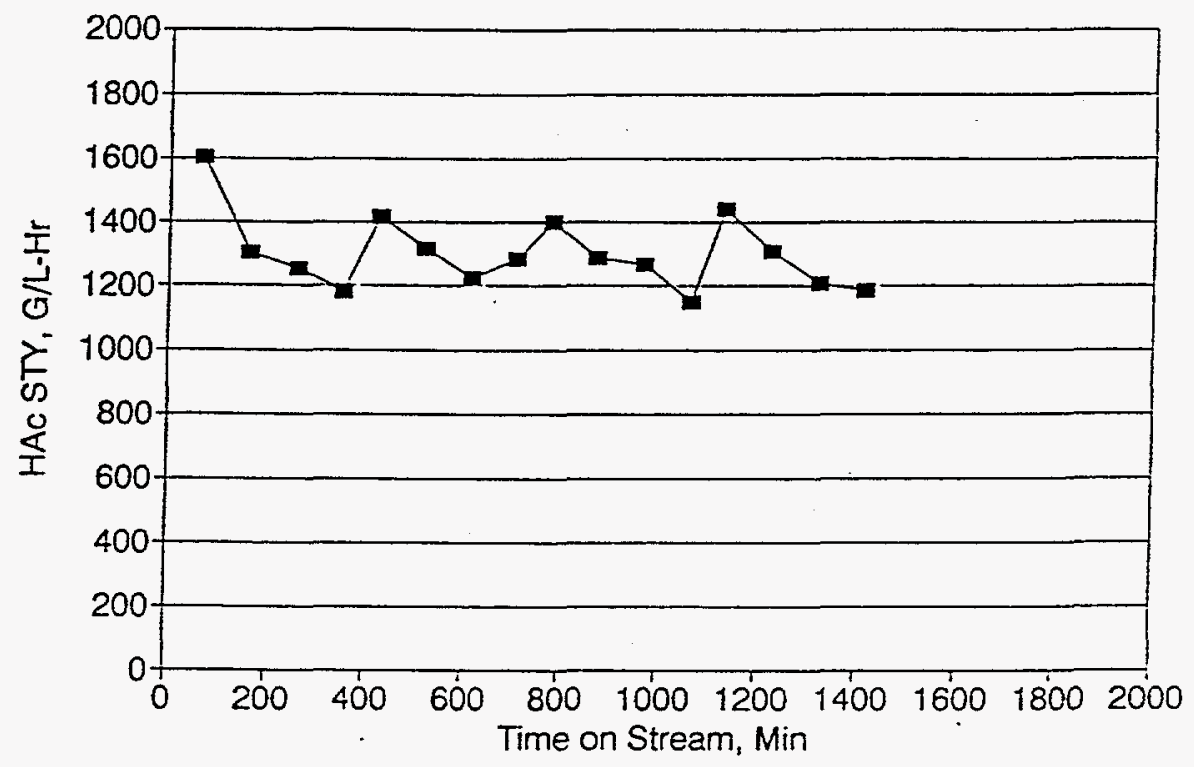

Figure 3.2.4 Ketene Conversion vs Time On Stream Pd Sponge

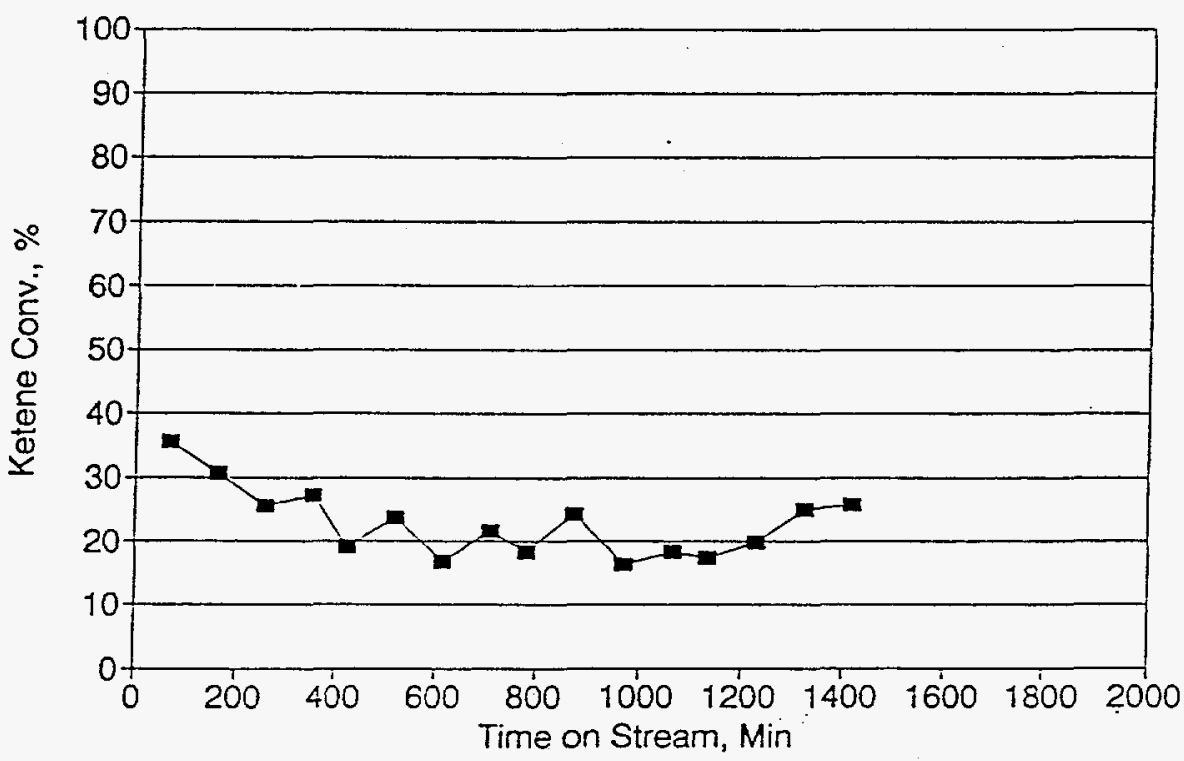


Figure 3.2.5 HAc Selectivity vs Time On Stream Pd Sponge

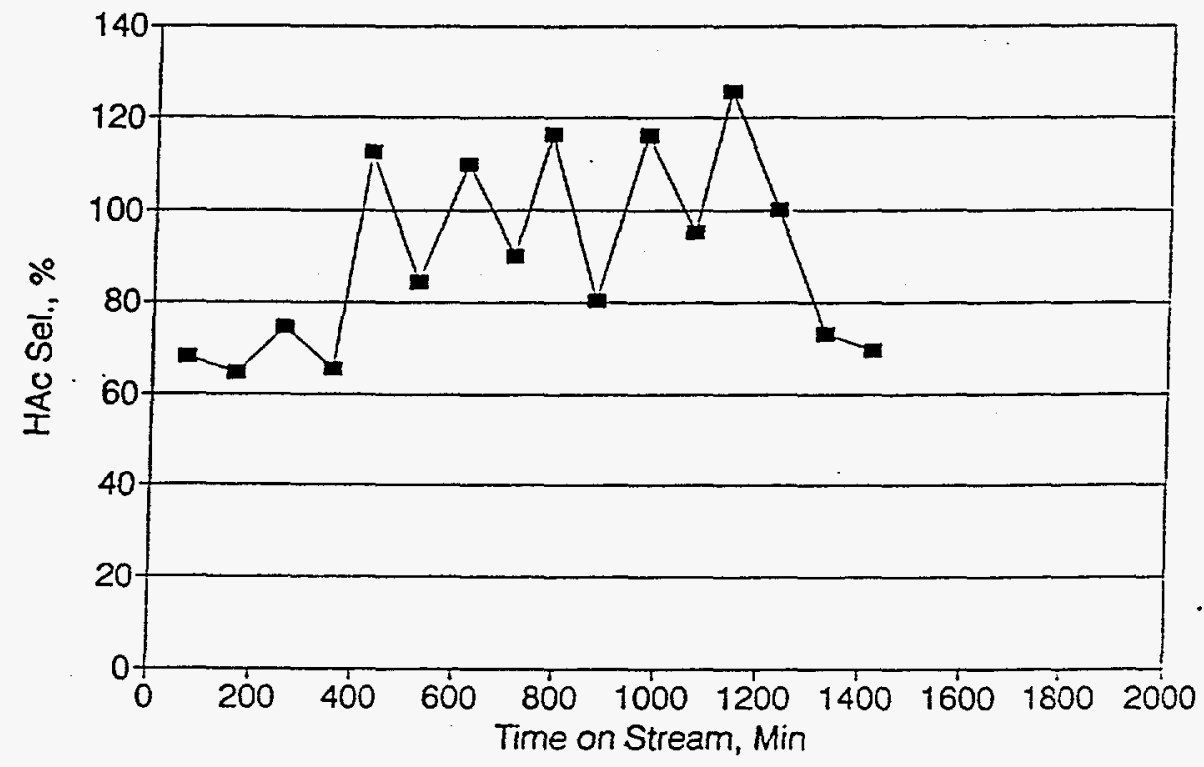

Figure 3.2.6 Ketene Accountability vs Time On Stream Pd Sponge

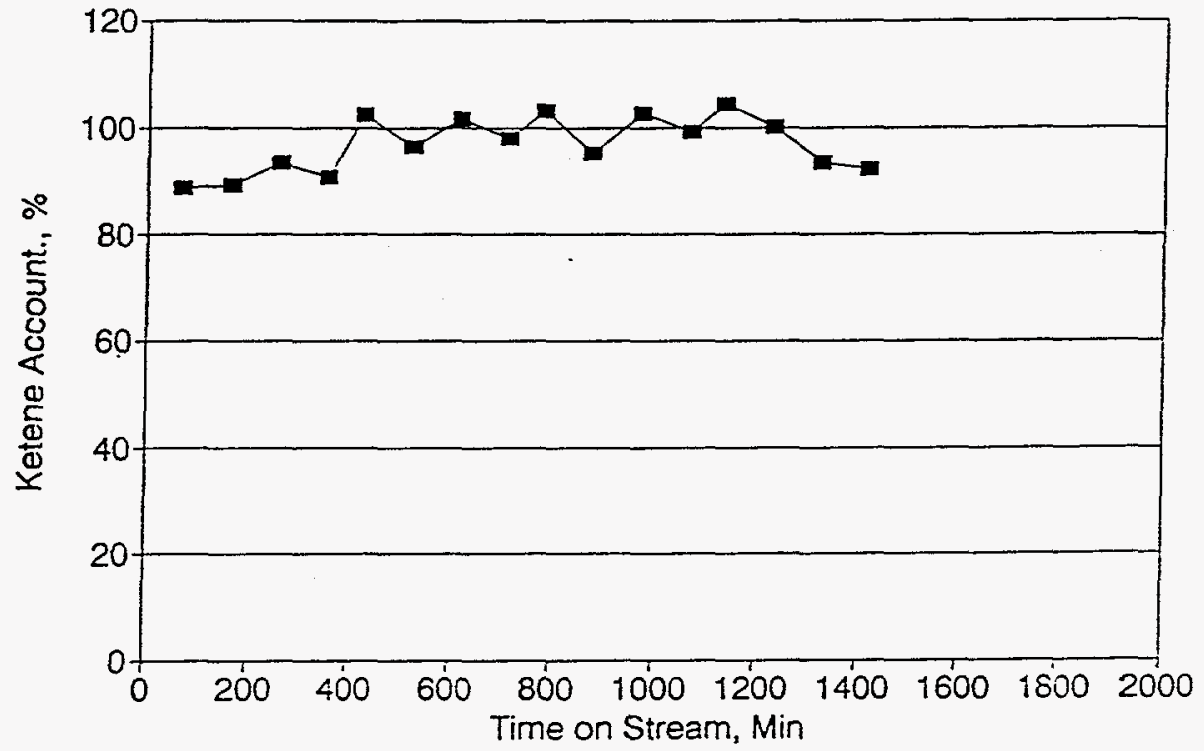


Over the last quarter, Eastman has realized this advance in VAM generation. Using a gasstripped reactor such as the one diagrammed in Scheme 4, Eastman has demonstrated a benchscale unit for the continuous conversion of acetaldehyde and ketene to VAM. Operationally, ketene generation and introduction was identical to that used in hydrogenation experiments, and acetaldehyde was also introduced as an approximately $20 \% \mathrm{AcH}$ vapor by bubbling nitrogen through a column of acetaldehyde maintained at $-20^{\circ} \mathrm{C}$. After combination of the ketene and acetaldehyde streams with a small stream of nitrogen, the mixture is fed to the reactor in Scheme 4. (The final feed composition is $6 \%$ ketene, $8 \% \mathrm{AcH}$, and $86 \%$ nitrogen.) The utilization of a gas-stripped reactor is important since this allows Eastman to strip VAM from the solution before VAM can undergo further reactions. Operating conditions and results of an early one week run, where several conditions were varied as we learned how to operate the system, are summarized below:

\section{Starting solution:}

$29.4 \mathrm{~g}$ starting mass

250 mmoles $\mathrm{Ac}_{2} \mathrm{O}$

27 mmoles HOAc

2.8 mmoles $\mathrm{PhSO}_{3} \mathrm{H}_{2} \mathrm{H}_{2} \mathrm{O}$ (benzenesulfonic acid monohydrate), later increased to 13.1 mmoles

\section{Conditions:}

operated for a total of 28.25 hours over a 5 day period

total ketene and acetaldehyde added over this period:

1187 mmoles ketene

1695 mmoles acetaldehyde

steam heat at $98^{\circ} \mathrm{C}$ or electric heat at $150^{\circ} \mathrm{C}$

most of the volatile product ( $\mathrm{HAc}, \mathrm{Ac}_{2} \mathrm{O}$, vinyl acetate) isolated from

the dry ice trap: no HOAc found in the dry ice trap!

Contents of heel solution after reaction:

$33.2 \mathrm{~g}$ recovered

6 mmoles HOAc

180 mmoles $\mathrm{Ac}_{2} \mathrm{O}$

72 mmoles EDA

Yield data:

total vinyl acetate (VAM generated/ketene fed X $100 \%$ ) yield $=59 \%$

total ketene accountability $=84 \%$

total acetaldehyde accountability $=103 \%$ 
Scheme 4. Gas Stripped Reactor for Direct Conversion of Ketene and Acetaldehyde to Vinyl Acetate

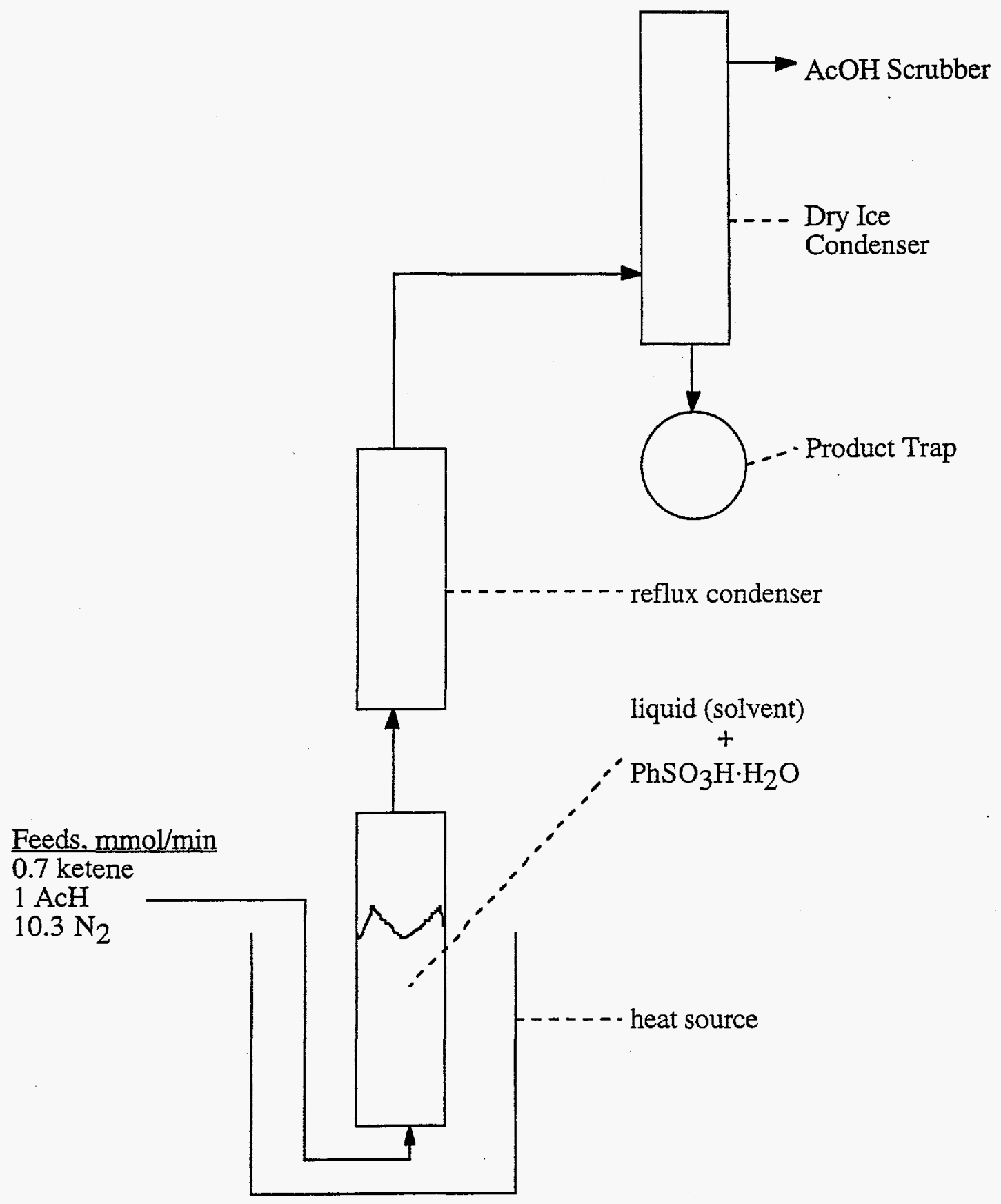


As can be seen from Table 3.2.4, key variables in this operation are the catalyst levels and operating temperatures. Initial studies using N-methyl pyrrolidinone (NMP, a cyclic tertiary amide) as the solvent rather than a mixture of acetic acid and acetic anhydride gave similar results.

Table 3.2.4. Effect of Catalyst Level and Temperature on VAM yields in the Gas-Stripped Reactor.

\begin{tabular}{ccc} 
mmol PhSO3H.H2O & $\frac{\text { temp. }{ }^{\circ} \mathrm{C}}{150}$ & yield vinyl acetate, \%* \\
\cline { 2 - 3 } 2.8 & 150 & 21 \\
13.1 & 98 & 78 \\
13.1 & 150 & 37 \\
13.1 & & 80
\end{tabular}

*yields $=$ VAM obtained/ketene fed X $100 \%$. These yields do not account for unreacted ketene and represent single pass yields.

As we have learned to operate this system and better close material balances, the vinyl acetate yields have steadily improved, and yields (as calculated above) are now in excess of $90 \%$. (The optimization studies are still underway and will be reported in more detail in the next quarterly report.) Further, although it does not significantly affect the design and operation of the unit, early observations indicate that, contrary to Eastman's original concept, the Bronsted acid catalyzed addition of ketene to acetaldehyde may actually proceed via a direct esterification of acetaldehyde to VAM without forming EDA as an intermediate.

Task 3.1.a. Preliminary Economics for a Ketene Based Process. Having identified operable processes at the bench scale for both the hydrogenation of ketene and the direct esterification of acetaldehyde with ketene, Eastman now has sufficient information to undertake preliminary economic estimates for the overall process. The conceptual flowsheet is in place. Eastman already has estimates for an appropriately sized acetic acid plant and ketene furnace, although the models will require some updating. Further, designs for the conversion of acetaldehyde and ketene to VAM are well underway. Preliminary estimates will not account for the available synergies between the components of the facility and will purposely be conservative.

\section{Future Directions}

General. Over the last quarter, Eastman has completed the construction of two additional ketene generation systems and has modified its existing reactor to allow faster, more reproducible, ketene introduction rates in both the hydrogenation and VAM generation processes. One is now operational and the second is currently being checked out. Additional efforts are being directed toward obtaining better, more reproducible methods for determining ketene levels. This is particularly critical since the largest variable in determining the efficiency of these reactions is the variability in measurements to obtain ketene balances. 
Task 1.1 Hydrogenation of Ketene. Over the next quarter Eastman will be:

a) pursuing a detailed study of the Pd catalysts, particularly in the presence of lower levels of diluent. (The goal is to approach the likely conditions in a plant environment.)

b) examining homogeneous catalysts and reactor designs for this hydrogenation.

Task 2.1 Conversion of Acetaldehyde and Ketene to VAM. Over the next quarter, Eastman expects to:

a) Continue its investigation of gas-stripped reactors and alternative designs for conducting these reactions.

b) Examine heterogeneous catalysts.

c) begin integrating the process with the ketene hydrogenation. (This will include an examination of a speculative direct conversion of ketene to vinyl acetate wherein the hydrogenation will be conducted in the presence of an acid catalyst.)

Task 3.1.a Preliminary Economics. Eastman expects to complete a preliminary estimate by the end of the second quarter of FY1996.

\section{Summary}

Over the last quarter Eastman has demonstrated that they could readily hydrogenate ketene to acetaldehyde, a reaction heretofore unknown. Eastman has also demonstrated that acetaldehyde could subsequently be recombined with ketene to generate VAM without the requirement for a separate acetic anhydride generation and reaction step. These form a very simple overall process for the generation of VAM. Eastman will optimize these processes over the next several months and will be generating initial cost estimates for the production of VAM from syngas by this method.

\section{Task 3.3 New Processes for Alcohols and Oxygenated Fuel Additives}

\subsubsection{Isobutanol Synthesis in a Three Phase System (RWTH Aachen)}

Fixed Bed Reactor Design and Runs

The optimal reaction conditions with $\mathrm{ZrO} / \mathrm{ZnO} / \mathrm{MnO}$ catalysts are presented below. Overall conditions were as follows:

$\begin{array}{ll}\text { Catalyst: } & \mathrm{ZrO} 2 / \mathrm{ZnO} / \mathrm{MnO} / \mathrm{K} / \mathrm{Pd}(\mathrm{pH} 9) \\ \text { GHSV: } & 23000 \mathrm{~h}-1 \\ \text { Reactor: } & \text { Fixed Bed }\end{array}$

The data have been summarized from 40 on-line measurements.

The mechanical stress of changes in pressure is much greater than that with changes in temperature, which leads to an increase in deactivation during pressure programming. This fact is borne out by the values at $25 \mathrm{MPa}$ which were measured initially. 
Figures 3.3.1 and 3.3.2 clarify that high pressures promote activities toward isobutanol and methanol production. The influence of temperature increases with increasing pressure. Increasing temperature itself decreases the yield of isobutanol and methanol. CO conversion increases nearly linearly with temperature and pressure (Figure 3.3.3). Selectivity to $\mathrm{CO}_{2}$ is not affected by pressure, but increases rapidly with higher temperatures (Figure 3.3.4).

\section{Figure 3.3.1 STY to Isobutanol vs Temperature and Pressure}

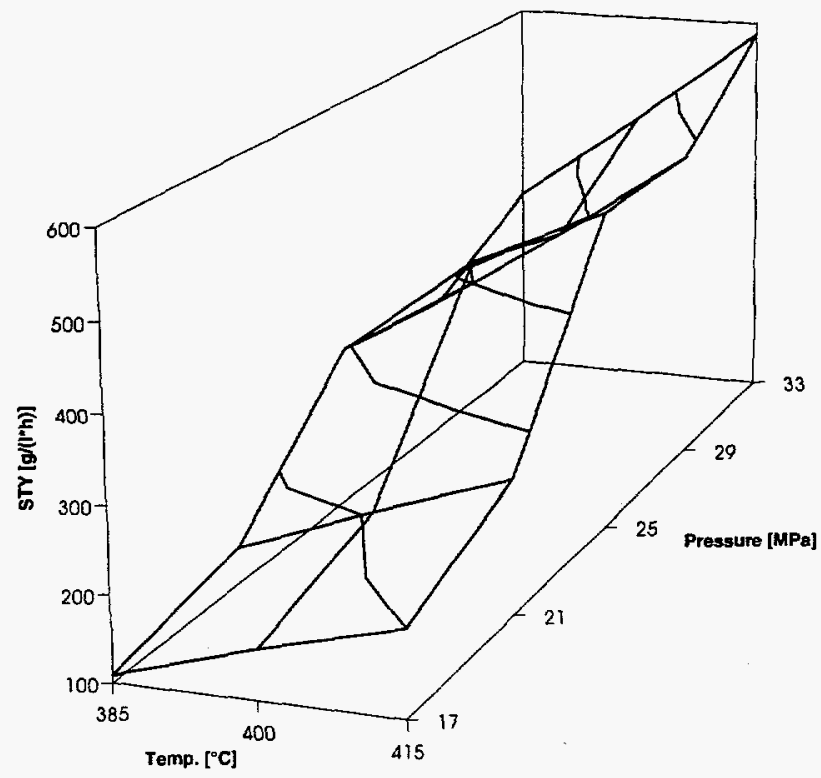


Figure 3.3.2 STY to Methanol vs Temperature and Pressure

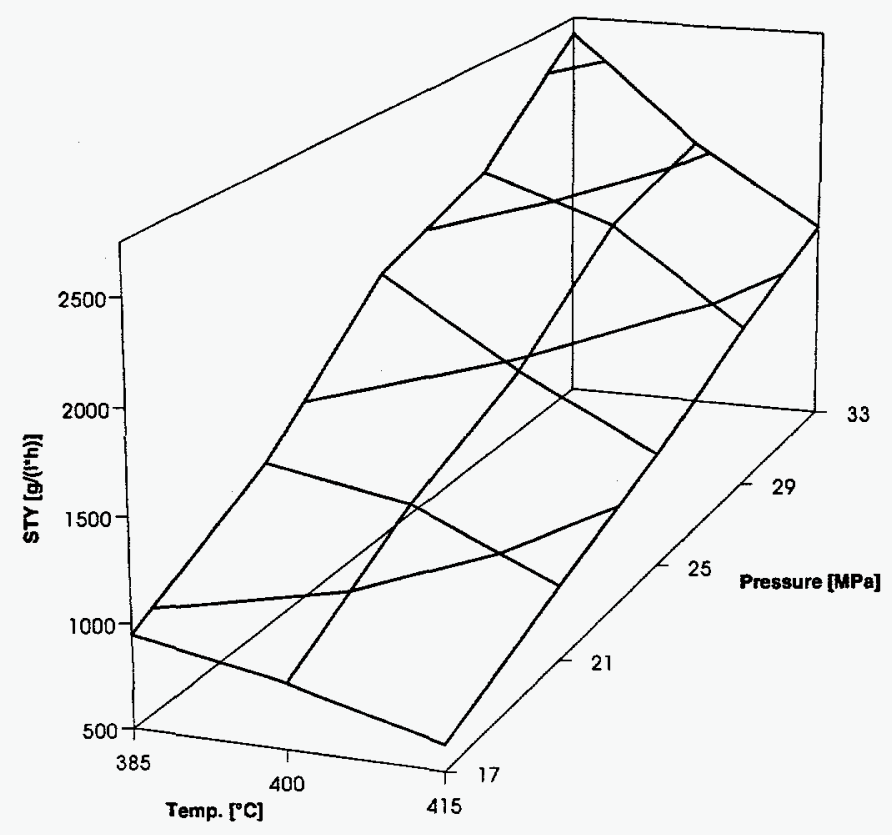

Figure 3.3.3 CO Conversion vs Temperature and Pressure

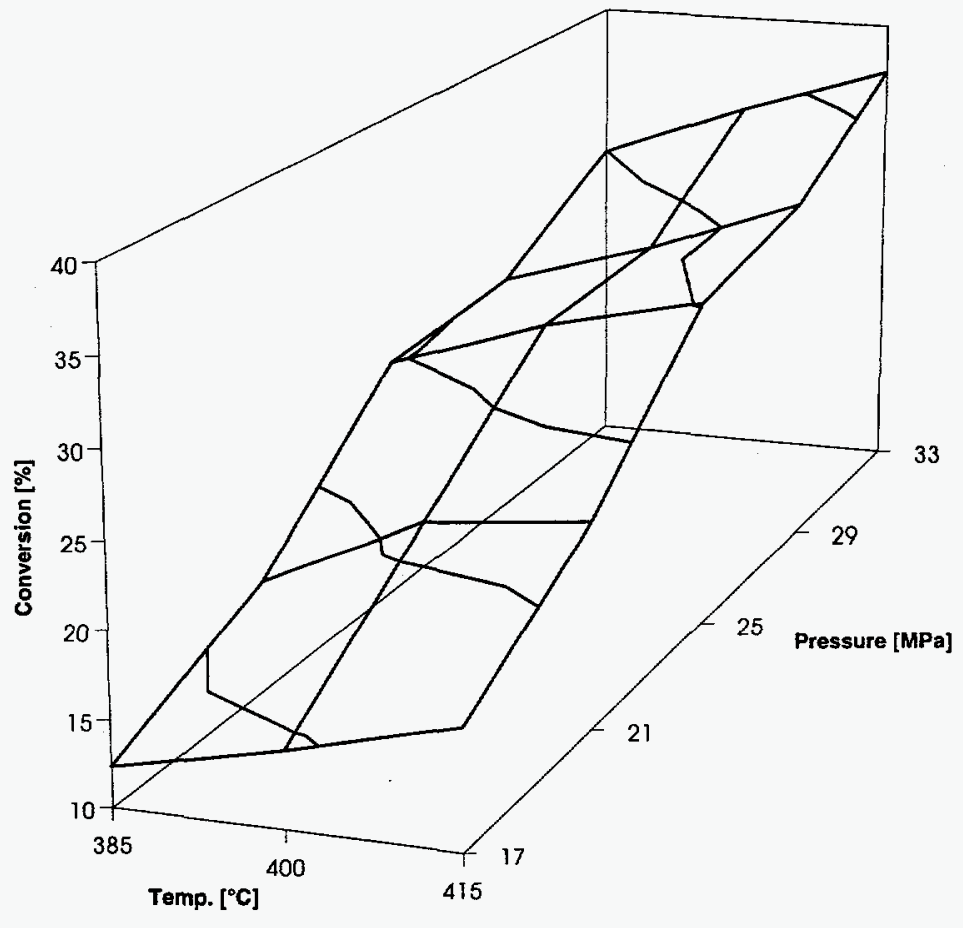


Figure 3.3.4 Selectivity to $\mathrm{CO}_{2}$ vs. Temperature and Pressure

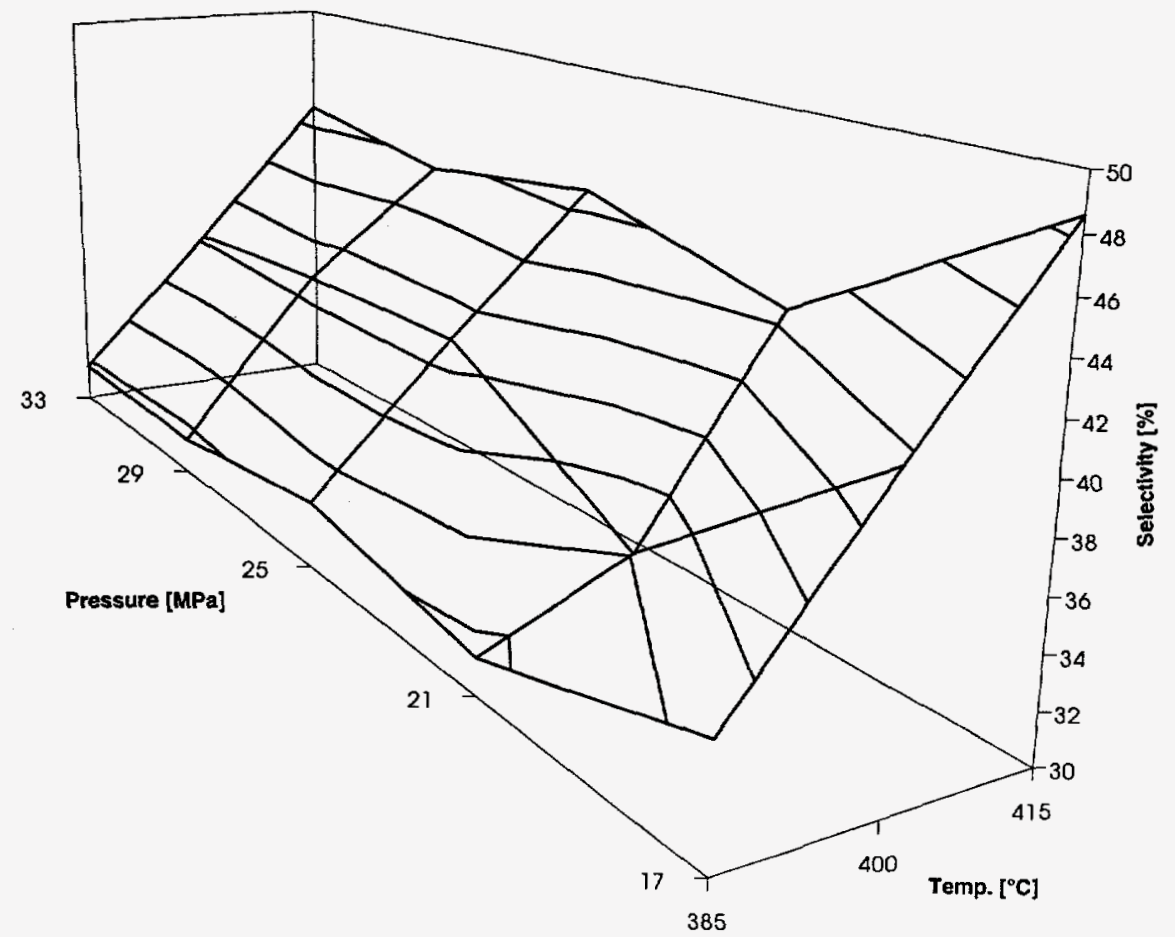

\section{Slurry Reactor Design and Runs}

Design

Over the last quarter, significant progress concerning the slurry reactor has been achieved. This progress concerns the theoretical estimation of the fundamental mass transfer coefficients as well as the results from runs.

It is well known that three limiting steps regarding methanol synthesis in a slurry system can exist ${ }^{1}$

1. mass transfer from the bulk gas phase to the bulk liquid

2. mass transfer from the bulk liquid to the boundary layer of the catalyst particle

3. reaction including pore diffusion to the active sites.

Concerning these steps in the isobutanol slurry system the following can be said:

1. The mass transfer coefficients klal for the step from the bulk gas to the bulk liquid phase were estimated with a correlation examined by Yagi and Yoshida ${ }^{2}$. For hydrogen and carbon monoxide, $\mathrm{k}_{1} \mathrm{a}_{1}$ coefficients are in the range from 0.7 to $3.3 \mathrm{~s}^{-1}$. These values are

${ }^{1} \mathrm{~N}$. H. Sweed: Intraparticle and interphase transport resistances in gas-liquid-solid catalytic reactors. In: A. E. Rodrigues, J. M. Calo, N. H. Sweed/W. Kotowski, J. Kliniec, Z. Kasprowicz: Int. Congress CHISA, Praha, Sept. 1984, S.1.

${ }^{2}$ H. Yagi, F. Yoshida: Gas Absorption by Newtonian and Non-Newtonian Fluid in Sparged Agitated Vessels, Ind. Eng. Chem. Proc. Des. Dev. 14, 488 (1975). 
a little higher for methanol synthesis due to the low surface tension of decalin ${ }^{3}$. Based on these values, a mass transfer limitation at this step is unlikely.

2. The volumetric mass transfer coefficient $\mathrm{k}_{\mathrm{S}} \mathrm{a}_{\mathrm{S}}$ for step 2 in the isobutanol slurry system is between 30 and $300 \mathrm{~s}^{-1}$, depending on component and particle size. These values of $k_{\mathrm{S}} \mathrm{a}_{\mathrm{S}}$ are 1-3 orders of magnitude higher then the coefficients $\mathrm{k}_{1} \mathrm{a}_{1}$ for step 1 . Thus, the mass transfer from the bulk liquid to the boundary layer of the catalyst particle as a limiting step can be ignored.

3. Assuming an isothermal catalyst particle, it is unlikely that step 3 will be rate-controlling for a catalyst particle smaller than $0.2 \mathrm{~mm}$ in the slurry bed (compared to a catalyst particle of $1 \mathrm{~mm}$ in diameter in a fixed bed reactor). The catalyst particles used in the slurry reactor were always crushed smaller then $0.2 \mathrm{~mm}$.

To prove the mathematical correlations, a run using the CSTR containing no inert oil was conducted. After eight on-line measurements, decalin was added using a high-pressure pump directly into the reactor up to the level of normal slurry runs. Subsequently, a second set of eight measurements was started. The results with and without decalin were nearly identical. This leads to the conclusion that synthesis is not affected by decalin at normal reaction conditions.

\section{Runs: Comparison of CSTR to Fixed Bed Reactor}

Measurement of actual catalyst activities with the slurry reactor was possible only by installing a preheater upstream of the slurry reactor. The preheater was filled with $10.5 \mathrm{ml}$ glass particles and heated to $400^{\circ} \mathrm{C}$. This arrangement seemed to cause adsorption of catalyst poisons at the surface of the glass particles instead on the active site of the catalysts. The effect was the same as seen in the fixed bed runs.

As shown in Table 3.3.1, STYs towards alcohols are quite similar but more methanol is produced in the slurry reactor. This might be an effect of temperature (see quarterly report 02/95).

\section{Table 3.3.1 Comparison Of Results In Two Different Reactors At Similar Reaction} Conditions

\begin{tabular}{c|cc} 
& slurry reactor & fixed bed reactor \\
\hline STY isobutanol & 204 & 370 \\
{$\left[\mathrm{~g} /\left(1^{*} \mathrm{~h}\right)\right]$} & & \\
STY methanol $\left[\mathrm{g} /\left(1^{*} \mathrm{~h}\right)\right]$ & 1057 & 738 \\
isobutanol/methanol & -0.19 & -0.50 \\
Conversion CO [\%] & 23 & 37 \\
Selectivity CO2 [\%] & 54 & 57 \\
GHSV [h-1] & 16900 & 17600 \\
Temperature: $415^{\circ} \mathrm{C}$, Pressure: $25 \mathrm{MPa}$, Catalyst: BASF-ZnO/Cr2O3/K
\end{tabular}

${ }^{3}$ S. Ledakowicz, H. Nettelhoff, W.-D. Deckwer: Gas-liquid mass transfer in a stirred autoclave reactor. In: Ind. Eng. Chem. Fundam., Bd. 23, 1984, S. 510/S. A. Miller, A. Ekstrom: Solubility and mass-transfer coefficients for hydrogen and carbon monoxide in n-octacosane. In: J. Chem. Eng. Data, Bd. 35, 1990, S. 125. 
Solvent

Following a publication by Kotowski et al., undecanol has been tested to obtain an alternative for decalin 4 . Undecanol was not found to be stable.

\section{Catalyst Screening}

To supplement the description of the second isobutanol unit in the last quarterly report, additional details are given here. The oven has been equipped with a small ventilator for minimizing the gradient of temperature. The cooling system used in this unit is composed of one condenser ( 1.2 meters) cooled to temperatures $<-30^{\circ} \mathrm{C}$ and two cooling traps cooled to temperatures of $-78^{\circ} \mathrm{C}$. With this system, condensation of nearly the total product amount is possible.

As in "Isobutanol Unit I," gas samples are collected and analyzed quantitatively. The first catalytic tests have been performed successfully and the first alkaline impregnated catalysts have been screened (see report 02/95).

\section{Test Routine for Catalyst Screening}

The first information that should be presented here is a suitable and comparable test routine. The difficulties with this are the strong variations in weight and volume of different catalysts. Therefore the catalyst screening will be constant in GHSV and all STYs will be given in $\mathrm{g} / \mathrm{kg}$ [cat].h-1. Additionally, all important data for catalyst comparison are collected. Reduction is performed as follows:
Gas:
$\mathrm{H}_{2}$
Pressure: $\quad 30$ bar
GHSV: $\quad 10000 \mathrm{~h}^{-1}$
Temperature: rising from 20 to $350^{\circ} \mathrm{C}$
Heating rate: $4 \% \mathrm{~min}$

After $350^{\circ} \mathrm{C}$ is reached, the reaction medium is changed from hydrogen to syngas:
Gas:
$\mathrm{CO} / \mathrm{H}_{2}=1$
Pressure:
250 bar
GHSV:
$20000 \mathrm{~h}^{-1}$
Temperature: 2 or 3 setpoints between 385 and $430^{\circ} \mathrm{C}$
Heating rate: $4 \% \mathrm{~min}$

Before sampling, the catalyst is allowed to come to steady state at each setpoint $(>1 \mathrm{~h})$. This modified reduction procedure has been chosen to save time in the test routine In addition, contact of the catalyst with syngas between 200 and $350^{\circ} \mathrm{C}$ is avoided. A comparison of the two different reduction procedures will be reported later.

\footnotetext{
${ }^{4}$ W. Kotowski, S. Ledakowicz, R. Zarzycki, L. Nowicki, H. Berndt: Methanol-Synthese an einem suspentierten CuO-ZnO-Mn2O3-Katalysator-Kopplung der Erzeugung von Methanol und Energie. Chem-Ing.-Tech. 67 (1995) Nr. 1, S. 80-82.
} 


\section{Results}

In the last quarterly report the section "Alkaline Free Catalysts" dealt with $\mathrm{ZrO} 2 / \mathrm{ZnO} / \mathrm{MnO}$ type catalysts washed free of alkaline metals. The intention is impregnation of these catalysts with alkaline and/or transition metals in order to obtain information about the influence of these components. The first members of this catalyst type have now been tested, and the main data are presented in Table 3.3.2.

Table 3.3.2 Screening Results

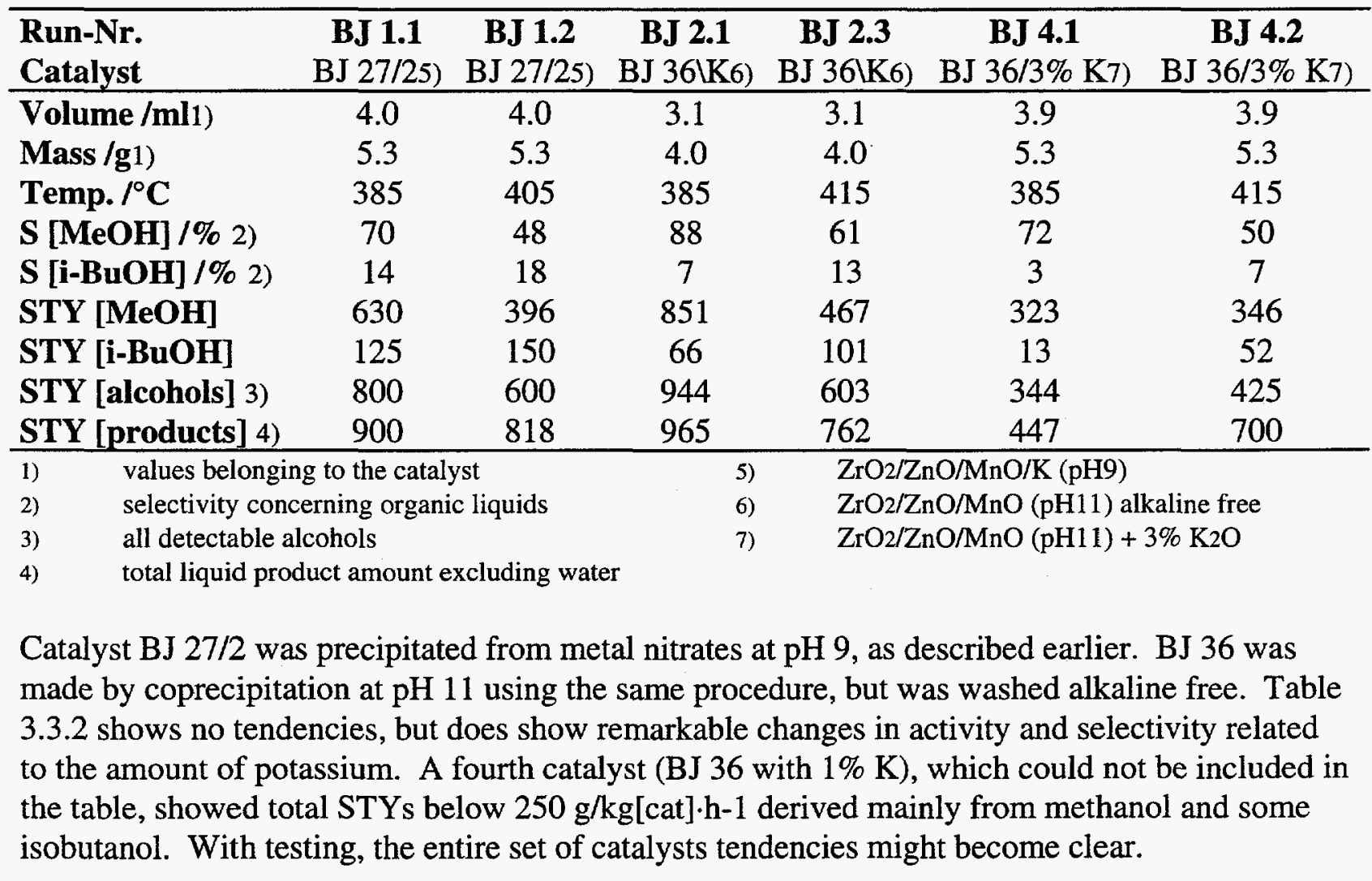

\section{Catalyst Preparation}

Among the currently used strategies to elucidate the scope of the $\mathrm{Zr} / \mathrm{Zn} / \mathrm{Mn}$-oxide system, the use of different synthesis methods was envisaged. For this purpose the following preparation methods were chosen:

- Coprecipitation

- Complexation

- Sol-Gel Methods

- Impregnation

In this quarter synthesis was mainly concentrated on the use of impregnation techniques and the optimization of sol-gel methods for our system. 


\section{Sol-Gel Based Catalysts}

As described earlier (report 02/95, 03/95), the sol-gel method involves the formation of an inorganic oxide network by controlled hydrolysis of suited metal precursors. The objective of this study is to develop synthesis methods that lead to homogeneous distributions of zirconia, zinc and manganese oxides. This is done by introducing zinc and manganese salts during the polymerization of zirconium alkoxides. With the proper use of stabilizing agents, the polymerization reactions can be adjusted in order to obtain homogeneous, clear gels. Control of hydrolysis and condensation reaction rates can be influenced by the addition of complexing agents such as acetic acid or acetyl acetone. In addition one can differentiate between acid and base catalyzed sol-gel reactions. Under acidic conditions, hydrolysis occurs at a faster rate than does condensation, and the resulting gel is weakly branched. Condensation is accelerated relative to hydrolysis with increasing $\mathrm{pH}$. Thus, a base-catalyzed gel is highly branched and contains colloidal aggregates. In this way the pore structure (surface area, pore volume and pore size distribution) can be adjusted. The results are provided in Tables 3.3.33.3.5.

Table 3.3.3 Influence Of Acetic Acid On $\mathrm{Zr} / \mathrm{Zn} / \mathrm{Mn}-\mathrm{Gels}^{1}$

\begin{tabular}{llll}
\hline HOAc/Zr & H2O/Zr & Gel Time $^{2}$ & Morphology \\
\hline 1 & 10 & immediate & Precipitate \\
1.5 & 10 & immediate & Gel/Precipitate \\
1.75 & 10 & 2 min. 30 sec. & Gel/Precipitate \\
2 & 10 & 19 days & Gel \\
4 & 10 & 4 months & Gel \\
\hline
\end{tabular}

${ }^{1}$ General Procedure: Zirconium propylate (100 mmol,70 wt\% solution in n-propanol) was stirred with the appropriate amount of acetic acid for 60 minutes. Zinc and manganese nitrate (each 100 $\mathrm{mmol}$ ) were dissolved in $85 \mathrm{ml} \mathrm{n}$-propanol and added instantaneously.

${ }^{2} \mathrm{Gel}$ Time is defined as the time elapsed between mixing the reagents and the formation of a gel so that no vortex can be produced by the stirrer.

\section{Table 3.3.4 Influence Of Nitric Acid On $\mathrm{Zr} / \mathrm{Zn} / \mathrm{Mn}-\mathrm{Gels}^{1}$}

\begin{tabular}{llll}
\hline HNO3/Zr & $\mathrm{H} 2 \mathrm{O} / \mathrm{Zr}$ & Gel Time & Morphology \\
\hline 1.33 & 10 & 28 sec. & $\mathrm{Gel}$ \\
1.56 & 10 & 13 min. 25 sec. & $\mathrm{Gel}$ \\
1.78 & 10 & 10 hours & $\mathrm{Gel}$ \\
\hline
\end{tabular}

${ }^{1}$ General Procedure: Zirconium propylate (100 mmol,70 wt $\%$ solution in n-propanol) was stirred with the appropriate amount of nitric acid for 60 minutes. Zinc and manganese nitrate (each 100 mmol) were dissolved in $85 \mathrm{ml} \mathrm{n}$-propanol and added instantaneously. 
Table 3.3.5 Influence Of Acetylacetone On $\mathrm{Zr} / \mathrm{Zn} / \mathrm{Mn}-\mathrm{Gels}^{1}$

\begin{tabular}{lllll}
\hline HNO3/Zr & acac/Zr & $\mathrm{H} 2 \mathrm{O} / \mathrm{Zr}$ & Gel Time & Morphology \\
\hline 0 & 0.25 & 10 & immediate & Precipitate \\
1.11 & 0.25 & 10 & $25 \mathrm{sec.}$ & Gel/Precipitate \\
1.33 & 0.25 & 10 & $30 \mathrm{~min}$. & Gel/Precipitate \\
1.56 & 0.25 & 10 & 11 days & Gel \\
\hline
\end{tabular}

${ }^{1}$ General Procedure: Zirconium propylate (100 mmol,70 wt $\%$ solution in $\mathrm{n}$-propanol) was stirred with the appropriate amount of nitric acid and acetylacetone for 60 minutes. Zinc and manganese nitrate (each $100 \mathrm{mmol}$ ) were dissolved in $85 \mathrm{ml}$-propanol and added instantaneously.

The data clearly show the influence of the stabilizing agents on gel time and morphology. In some cases initially a clear gel is obtained, which becomes cloudy after several hours. This is caused by prolonged condensation processes with aging. The wet gels were transformed into aerogels via supercritical drying in an autoclave. Analyses obtained so far indicate that this method leads to formation of mesoporous materials with surface areas ranging from 80-130 $\mathrm{m}^{2} / \mathrm{g}$. XRD analysis indicated the formation of crystalline zinc oxide. In a separate experiment (Table 3.3.6), it was found that this phase is formed during the supercritical drying step.

The influence of the supercritical drying step and the manner of addition of the zinc and manganese salts were tested with acetic acid as the stabilizing agent (Table 3.3.6). In the above described experiments, the water needed for gel formation was introduced as the crystal water contained in the metal salts. The influence of the metal salts upon gel formation was investigated by producing a gel first and mixing it with the metal salts afterward, followed by a supercritical drying step. The BET data show that the latter method leads to a compound that has a slightly smaller surface area (101 vs. $113 \mathrm{~m}^{2} / \mathrm{g}$ ); however, the pore size is larger ( 70 vs. $55 \AA$ ). The influence of the drying step becomes evident by comparing an ordinarily dried gel with a supercritically dried one. The xerogel produced has a very low surface area, and it appears that supercritical drying is needed to obtain large surface areas.

Table 3.3.6 Influence of Post Treatment and Introduction of Metal Precursors

\begin{tabular}{llllll}
\hline HOAc/Zr & H2O/Zr & Gel Time & $\begin{array}{l}\text { Post } \\
\text { Treatment }^{1}\end{array}$ & $\begin{array}{l}\text { BET } \\
(\mathrm{m} 2 / \mathrm{g})\end{array}$ & $\begin{array}{l}\text { Mean pore size } \\
(\AA)\end{array}$ \\
\hline 1.75 & 10 & 2 min. 30 sec. & xerogel & 17 & 40 \\
1.75 & 10 & 2 min. 30 sec. & aerogel & 113 & 55 \\
1.75 & $4+10$ & 1 min. & aerogel & 101 & 70 \\
\hline
\end{tabular}

${ }^{1}$ The xerogel was dried by evaporating the solvent in a rotavap. Subsequently the powder was dried in an oven at $120^{\circ} \mathrm{C}$ for $16 \mathrm{hrs}$. The aerogel was prepared as follows: after dispersing the gel with n-propanol (200 $\mathrm{ml}$ ), the autoclave was flushed with nitrogen and pressurized with 50 bar nitrogen. Then the autoclave was heated to $280^{\circ} \mathrm{C}\left(1^{\circ} \mathrm{C} / \mathrm{min}\right.$.). After keeping this conditions $\left(280^{\circ} \mathrm{C}\right.$, ca. 140 bar) constant for 30 minutes, the autoclave was vented slowly (ca. $1 \mathrm{bar} / \mathrm{min}$.). In all cases the powders obtained were calcined at $400^{\circ} \mathrm{C}$. 


\section{Impregnation Techniques}

The observed synergistic effect of zinc and manganese oxide on isobutanol production prompted an investigation of the influence of different impregnation sequences on catalytic behavior. For this purpose, two different zirconia supports were prepared according to a precipitation and a solgel method, as shown in Table 3.3.7.

\section{Table 3.3.7 Analytical Data of Zirconia Supports ${ }^{1}$}

\begin{tabular}{lll}
\hline & BET $(\mathrm{m} 2 / \mathrm{g})$ & Mean pore size $(\AA)$ \\
\hline Precipitation $\left(\mathrm{LiOH}, 80^{\circ} \mathrm{C}, \mathrm{pH} 9\right)$ & 275 & 35 \\
Sol-Gel $(\mathrm{HOAc} / \mathrm{Zr} 1.75 ; \mathrm{H} 2 \mathrm{O} / \mathrm{Zr} 4)$ & 159 & 65 \\
\hline
\end{tabular}

${ }^{1}$ Both supports were calcined at $400^{\circ} \mathrm{C}$.

These supports were impregnated in three different modes. The first method involved a primary impregnation with zinc acetate followed by a secondary impregnation with manganese acetate. The second method followed the reverse route-impregnating first with manganese and then with zinc. In the third method, mixtures of zinc- and manganese-acetate were used. All impregnations were conducted so as to obtain final loadings of $10 \mathrm{~mol} \%$, respectively, of zinc and manganese. Between subsequent impregnations, the samples were calcined at $400^{\circ} \mathrm{C}$.

The use of deposition-precipitation techniques (selective precipitation of precursors on supports) is also being examined.

\subsubsection{Oxygenates via Synthesis Gas (Lehigh University)}

\section{Overall 4QFY95 Objectives}

(i) Continue studies of increasing the conversion of $\mathrm{H}_{2} / \mathrm{CO}$ to higher alcohols by promotion of the $\mathrm{C}_{1} \rightarrow \mathrm{C}_{2}$ carbon chain growth step over Cs-promoted $\mathrm{Cu} / \mathrm{ZnO} / \mathrm{Cr}_{2} \mathrm{O}_{3}$ and $\mathrm{MoS}_{2}$ catalysts,

(ii) Enhance the $\mathrm{C}_{2} \rightarrow \mathrm{C}_{3} \rightarrow \mathrm{C}_{4}$ carbon chain growth steps over $\mathrm{Cs} / \mathrm{Cu} / \mathrm{ZnO} / \mathrm{Cr}_{2} \mathrm{O}_{3}$ catalysts, and

(iii) Prepare and test high surface area $\mathrm{Cu} / \mathrm{ZrO}_{2}$ catalysts, both Cs-doped and undoped, that are candidates for the synthesis of $\mathrm{C}_{1}-\mathrm{C}_{5}$ alcohols, in particular branched products such as isobutanol.

\section{Results and Discussion $\mathrm{Cu} / \mathrm{ZrO} \mathrm{O}_{2}$ Catalysts}

Zirconia-based catalysts are being investigated for their potential as alcohol synthesis catalysts, and a series of $\mathrm{CuO} / \mathrm{ZrO}_{2}$ catalysts having different $\mathrm{Cu} / \mathrm{Zr}$ molar ratios were previously prepared by aqueous coprecipitation at constant $\mathrm{pH}$ and temperature as described in quarterly progress report no. 2. 
A fresh sample of the $\mathrm{CuO} / \mathrm{ZrO}_{2}=10 / 90 \mathrm{~mol} \%$ catalyst $(2 \mathrm{~g}$ mixed with Pyrex beads for dilution) was loaded into the reactor after being calcined in air at $350^{\circ} \mathrm{C}$. It was then reduced at $250^{\circ} \mathrm{C}$ using a constant flow of $2 \mathrm{vol} \% \mathrm{H}_{2} / \mathrm{N}_{2}$ gas mixture at atmospheric pressure; the formation of $\mathrm{H}_{2} \mathrm{O}$ during the reduction process was monitored by gas chromatography. After the reduction was complete, the reactor was cooled to ambient temperature, the gas flow was changed to $\mathrm{H}_{2} / \mathrm{CO}=70 / 30 \mathrm{~mol} \%$ synthesis gas, and the reactor was pressurized to $7.6 \mathrm{MPa}$. After the gas hourly space velocity (GHSV) was verified as $6120 \mathrm{l} / \mathrm{kg}$ cat $/ \mathrm{hr}$, the temperature was increased to $250^{\circ} \mathrm{C}$, and steady-state testing was carried out. The 70/30 composition satisfies the stoichiometric condition for product formation.

After the above experiment, the quantity of $\mathrm{CO}_{2}$ in the $\mathrm{H}_{2} /\left(\mathrm{CO}+\mathrm{CO}_{2}\right)=70 / 30 \mathrm{~mol} \%$ synthesis gas was systematically varied from $0 \%$ to $30 \%$ by using the following gas mixtures:

$$
\begin{array}{cc}
\mathrm{H}_{2} / \mathrm{CO} / \mathrm{CO}_{2}= & 70 / 30 / 0 \\
& 70 / 28 / 2 \\
& 70 / 25 / 5 \\
& 70 / 20 / 10 \\
& 70 / 15 / 15 \\
& 70 / 10 / 20 \\
& 70 / 5 / 25 \\
& 70 / 2 / 28 \\
& 70 / 0 / 30
\end{array}
$$

The gas mixtures were changed while the reaction temperature and pressure were maintinaed at the constant preset values. Preliminary results showed that the presence of $\mathrm{CO}_{2}$ in the synthesis gas steadily promoted the formation of methanol while inhibiting the formation of dimethylether (DME), as shown in Figures 3.3.5 and 3.3.6.

After testing with the sequence of increasing $\mathrm{CO}_{2}$ concentration was completed, the reactant mixture was changed to the original $\mathrm{CO}_{2}$-free $\mathrm{H}_{2} / \mathrm{CO}=2.33$, and a slightly lower catalytic activity was noted. The productivities of both methanol and dimethylether (DME) were somewhat lower than the initially observed space time yields. However, with an increase in the $\mathrm{CO}_{2}$ content of the reactant again, reproducible catalyst behavior was observed, as shown in Figures 3.3.5 and 3.3.6. Therefore, no significant deactivation was observed during this testing with $\mathrm{CO}_{2}$ in the synthesis gas reactant mixture. 
Figure 3.3.5 Space Time Yields ( $\mathrm{g} / \mathrm{kg}$ catal/hr) of Methanol vs $\mathrm{CO}_{2}$ Content in the $\mathrm{H}_{2} /\left(\mathrm{CO}+\mathrm{CO}_{2}\right)=70 / 30 \mathrm{~mol} \%$ Synthesis Gas at $250^{\circ} \mathrm{C}$ and $7.6 \mathrm{MPa}$ with GHSV $=6,1201 / \mathrm{kg} \mathrm{catal} / \mathrm{hr}$

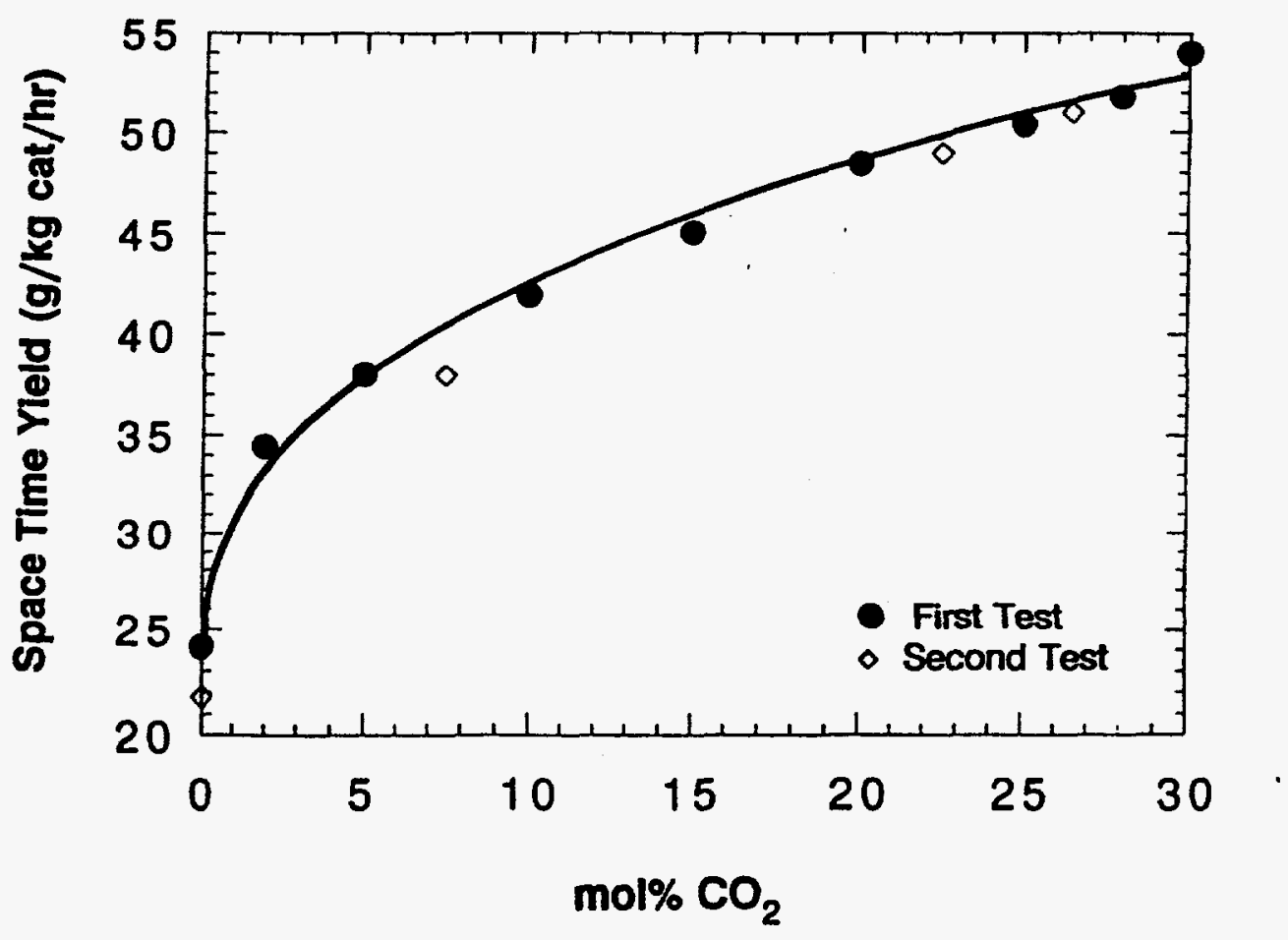


Figure 3.3.6 Space Time Yields ( $\mathrm{g} / \mathrm{kg}$ catal/hr) of Dimethylether vs the $\mathrm{CO}_{2}$ Content in the $\mathrm{H}_{2} /\left(\mathrm{CO}+\mathrm{CO}_{2}\right)=70 / 30 \mathrm{~mol} \%$ Synthesis Gas at $250^{\circ} \mathrm{C}$ and $7.6 \mathrm{MPa}$ with GHSV $=6,120 \mathrm{l} / \mathrm{kg} \mathrm{catal} / \mathrm{hr}$

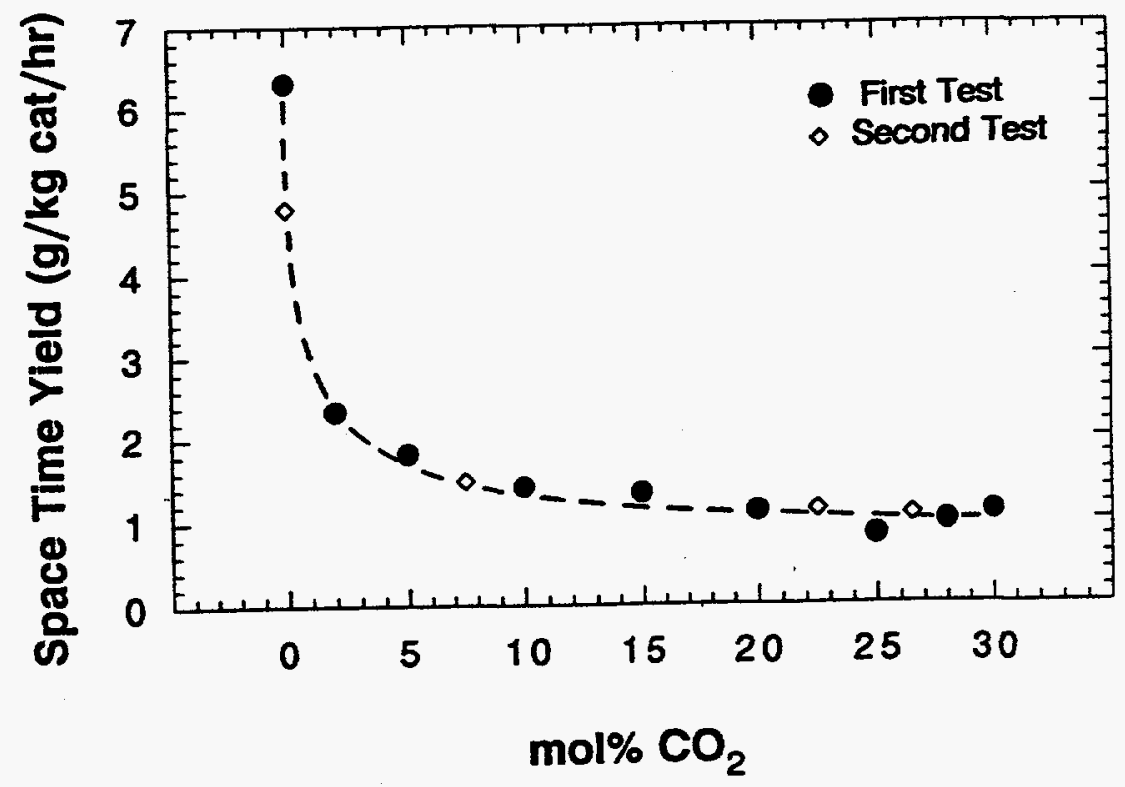


This continuous testing to determine the effect of $\mathrm{CO}_{2}$ on the catalytic behavior of the $\mathrm{Cu} / \mathrm{ZrO} \mathrm{O}_{2}=$ $10 / 90$ catalyst was carried out over two weeks. This same catalyst sample was tested further in studying the effect of reaction temperature and $\mathrm{H}_{2} / \mathrm{CO}$ synthesis gas ratio on the activity and productivity of the catalyst. The reaction temperature was varied from 250 to $330^{\circ} \mathrm{C}$ while a $\mathrm{H}_{2} / \mathrm{CO}=0.45$ synthesis gas was maintained at $7.6 \mathrm{MPa}$ with $\mathrm{GHSV}=5500 \mathrm{l} / \mathrm{kg} \mathrm{cat} / \mathrm{hr}$. The space time yields (STY) of methanol and dimethylether are shown in Figure 3.3.7. A significant increase in the space time yield of dimethylether (DME) was observed with increasing reaction temperature. The DME productivity ranged from $5 \mathrm{~g} / \mathrm{kg}$ cat $/ \mathrm{hr}$ at $250^{\circ} \mathrm{C}$ to $105 \mathrm{~g} / \mathrm{kg}$ cat $/ \mathrm{hr}$ at $330^{\circ} \mathrm{C}$. However, methanol space time yield remained approximately constant at $» 20 \mathrm{~g} / \mathrm{kg} \mathrm{cat} / \mathrm{hr}$, after a small initial increase from $16.5 \mathrm{~g} / \mathrm{kg} \mathrm{cat} / \mathrm{hr}$.

While the other reaction conditions were maintained, the $\mathrm{H}_{2} / \mathrm{CO}$ ratio was varied from 0.45 to 2.33 at a constant reaction temperature of $310^{\circ} \mathrm{C}$. Increasing the $\mathrm{H}_{2} / \mathrm{CO}$ ratio had a gradual effect on the space time yields of the products, as shown in Figure 3.3.8. The DME productivity slowly decreased from 60 to $44 \mathrm{~g} / \mathrm{kg}$ cat $/ \mathrm{hr}$ as the $\mathrm{H}_{2} / \mathrm{CO}$ ratio was increased. Methanol productivity exhibited a small increase from $22 \mathrm{~g} / \mathrm{kg}$ cat $/ \mathrm{hr}\left(\mathrm{H}_{2} / \mathrm{CO}=0.45\right)$ to $30 \mathrm{~g} / \mathrm{kg}$ cat $/ \mathrm{hr}\left(\mathrm{H}_{2} / \mathrm{CO}=1.50\right)$, but a further increase in the $\mathrm{H}_{2} / \mathrm{CO}$ ratio did not have an effect on the methanol space time yield.

$\mathrm{A} \mathrm{Cu} / \mathrm{ZrO}_{2}=30 / 70 \mathrm{~mol} \%$ catalyst was tested to determine if its catalytic behavior was similar to that of the $\mathrm{Cu} / \mathrm{ZrO}_{2}=10 / 90 \mathrm{~mol} \%$ catalyst. As with the $10 / 90 \mathrm{~mol} \%$ sample, the DME productivity showed a marked increase with temperature, as shown in Figure 3.3.9; the STY increased from $5 \mathrm{~g} / \mathrm{kg}$ cat $/ \mathrm{hr}$ at $250^{\circ} \mathrm{C}$ to $86 \mathrm{~g} / \mathrm{kg}$ cat $/ \mathrm{hr}$ at $330^{\circ} \mathrm{C}$. The space time yield for methanol was observed to increase from $17 \mathrm{~g} / \mathrm{kg}$ cat $/ \mathrm{hr}$ at $250^{\circ} \mathrm{C}$ to $34 \mathrm{~g} / \mathrm{kg}$ cat $/ \mathrm{hr}$ at $290^{\circ} \mathrm{C}$, but it then decreased slowly to $21 \mathrm{~g} / \mathrm{kg}$ cat $/ \mathrm{hr}$ at $330^{\circ} \mathrm{C}$. The trends observed in STY of the products with increasing $\mathrm{H}_{2} / \mathrm{CO}$ ratio were similar to those of the $10 / 90 \mathrm{~mol} \%$ catalyst, as indicated by comparing Figure 3.3.10 with Figure 3.3.8. The DME STY decreased slowly from $58 \mathrm{~g} / \mathrm{kg} \mathrm{cat} / \mathrm{hr}$ $\left(\mathrm{H}_{2} / \mathrm{CO}=0.45\right)$ to $44 \mathrm{~g} / \mathrm{kg} \mathrm{cat} / \mathrm{hr}\left(\mathrm{H}_{2} / \mathrm{CO}=2.33\right)$. The STY of methanol increased from $24 \mathrm{~g} / \mathrm{kg}$ cat $/ \mathrm{hr}\left(\mathrm{H}_{2} / \mathrm{CO}=0.45\right)$ to $38 \mathrm{~g} / \mathrm{kg}$ cat $/ \mathrm{hr}\left(\mathrm{H}_{2} / \mathrm{CO}=2.33\right)$. The increased $\mathrm{Cu}$ content of the $\mathrm{Cu} / \mathrm{ZrO}_{2}=30 / 70$ catalyst did not appear to significantly affect the observed catalytic behavior of the catalyst compared with the $\mathrm{Cu} / \mathrm{ZrO}_{2}=10 / 90$ catalyst.

BET surface areas of the tested catalysts were $72 \mathrm{~m}^{2} / \mathrm{g}$ for the $10 / 90$ mol\% sample (after »1 month of testing at $7.6 \mathrm{MPa}$ and various temperatures extending up to $330^{\circ} \mathrm{C}$ ) and $55 \mathrm{~m}^{2} / \mathrm{g}$ for the $30 / 70 \mathrm{~mol} \%$ sample. The corresponding values for the fresh, calcined catalysts were 148 and $78 \mathrm{~m}^{2} / \mathrm{g}$, respectively.

X-Ray diffraction (XRD) powder patterns of both $10 / 90$ and $30 / 70 \mathrm{~mol} \%$ tested catalysts did not show any peaks corresponding to crystalline copper. However, the tested $30 / 70 \mathrm{~mol} \%$ sample exhibited a sharp pattern assignable to crystalline zirconia. The fresh, calcined catalysts of both compositions were observed to be $\mathrm{X}$-ray amorphous. 
Figure 3.3.7 Effect of Reaction Temperature on the Productivity of Methanol and Dimethylether from $\mathrm{H}_{2} / \mathrm{CO}=0.45$ Synthesis Gas at 7.6 MPa and GHSV $=55001 / \mathrm{kg}$ cat $/ \mathrm{hr}$ over the $\mathrm{Cu} / \mathrm{ZrO}_{2}=10 / 90 \mathrm{~mol} \%$ Catalyst

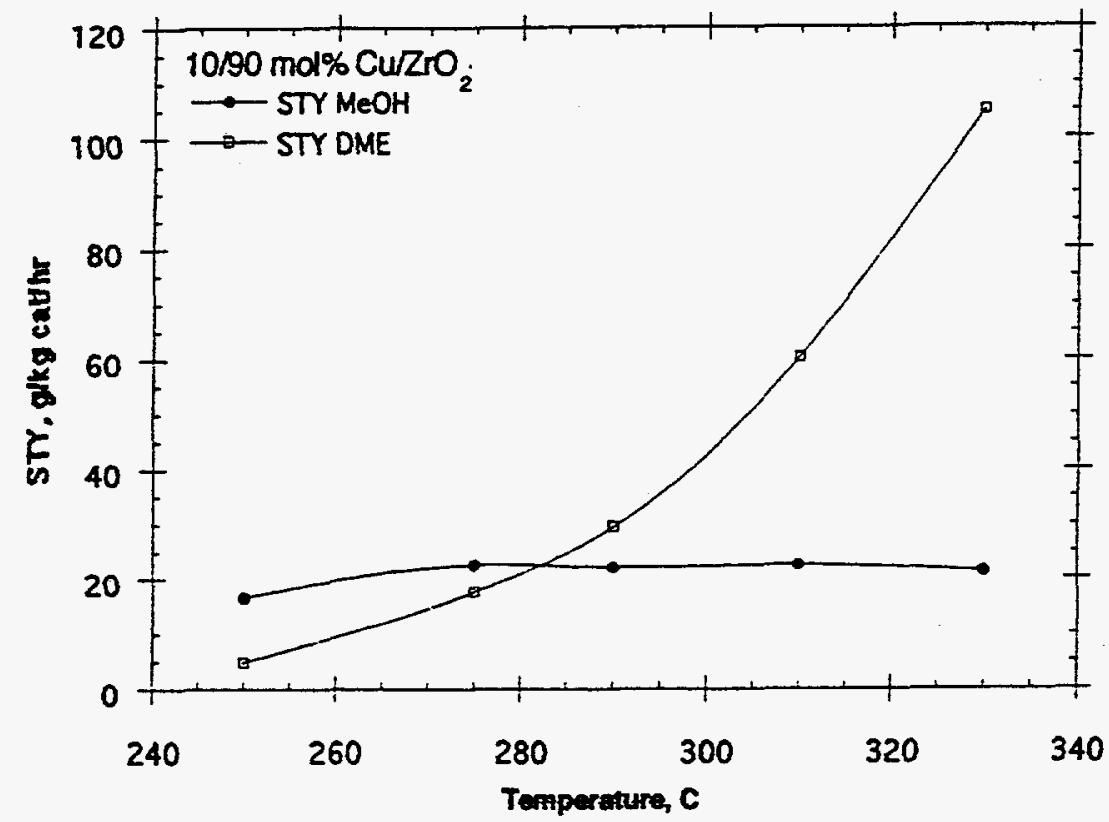

Figure 3.3.8 Effect of the $\mathrm{H}_{2} / \mathrm{CO}$ Synthesis Gas Ratio on the Productivity of Methanol and Dimethylether at $310^{\circ} \mathrm{C}, 7.6 \mathrm{MPa}$, and $\mathrm{GHSV}=5500 \mathrm{l} / \mathrm{kg} \mathrm{cat} / \mathrm{hr}$ over the $\mathrm{Cu} / \mathrm{ZrO}_{2}=10 / 90 \mathrm{~mol} \%$ Catalyst

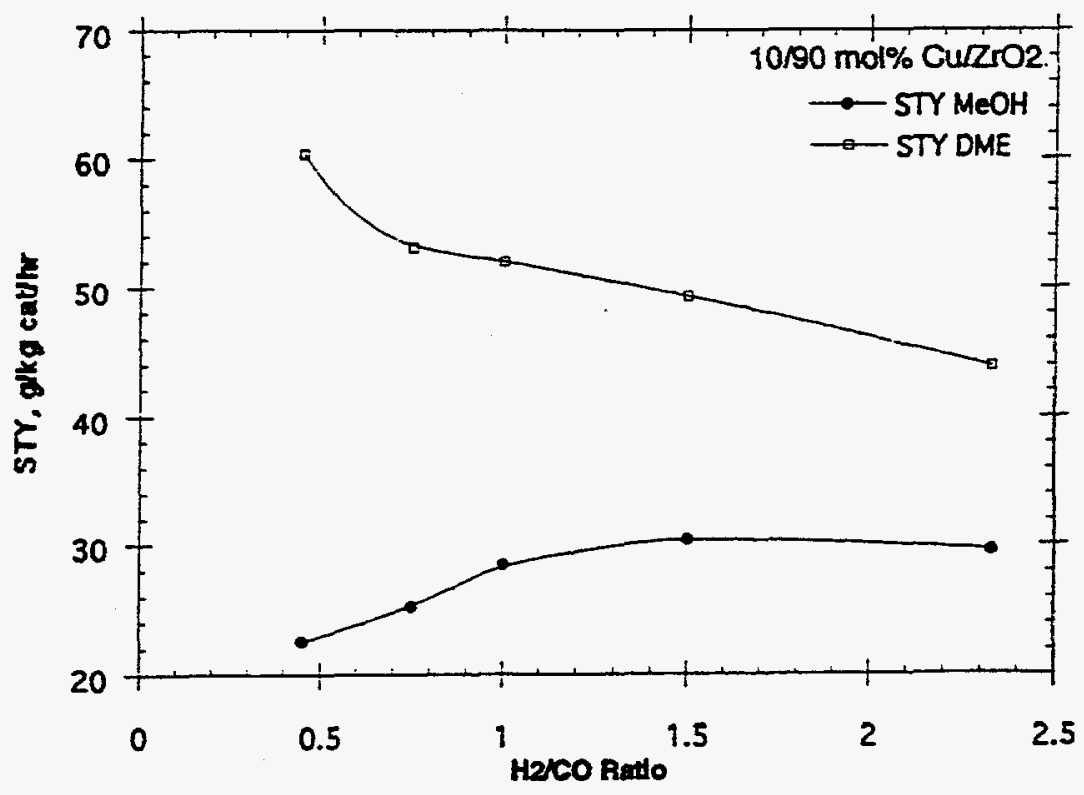


Figure 3.3.9 Effect of Reaction Temperature on the Productivity of Methanol and Dimethylether from $\mathrm{H}_{2} / \mathrm{CO}=0.45$ Synthesis Gas at 7.6 MPa and GHSV $=5500 \mathrm{l} / \mathrm{kg}$ cat $/ \mathrm{hr}$ over the $\mathrm{Cu} / \mathrm{ZrO}_{2}=30 / 70 \mathrm{~mol} \%$ Catalyst

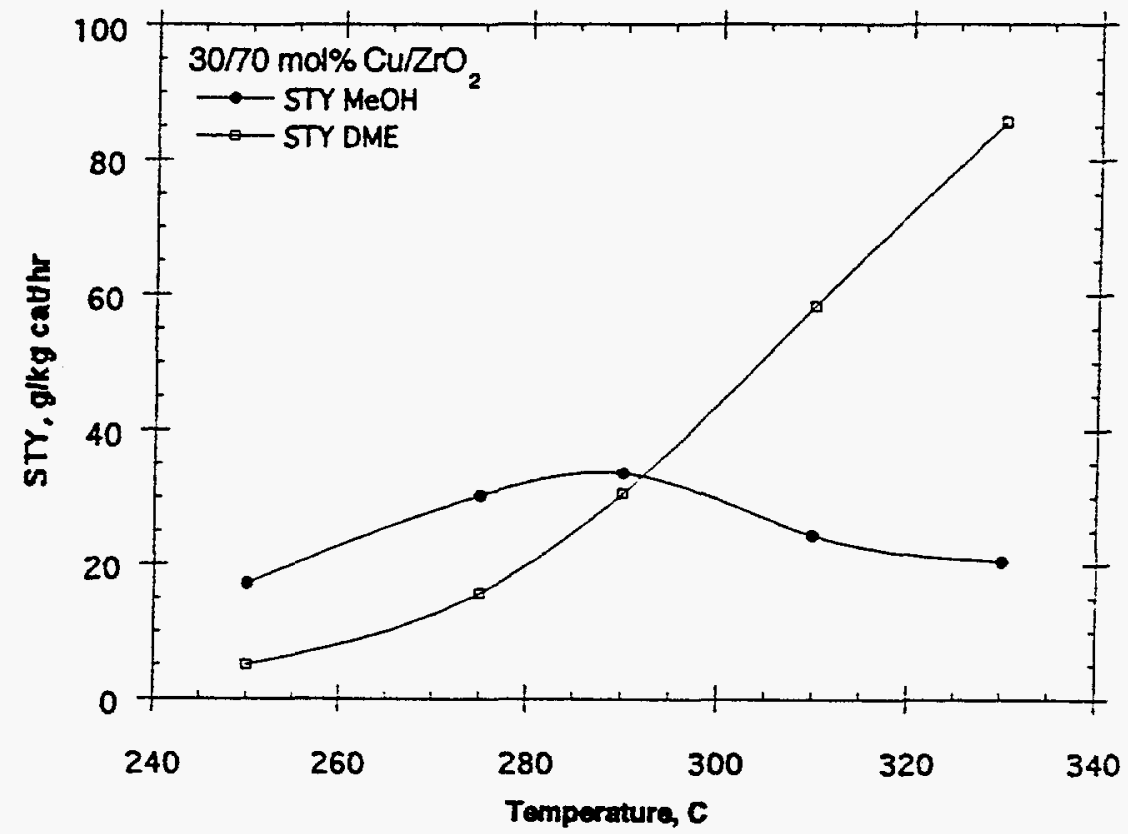

Figure 3.3.10 Effect of the $\mathrm{H}_{2} / \mathrm{CO}$ Synthesis Gas Ratio on the Productivity of Methanol and Dimethylether at $310^{\circ} \mathrm{C}, 7.6 \mathrm{MPa}$, and GHSV $=5500 \mathrm{I} / \mathrm{kg} \mathrm{cat} / \mathrm{hr}$ over the $\mathrm{Cu} / \mathrm{ZrO}_{2}=30 / 70 \mathrm{~mol} \%$ Catalyst

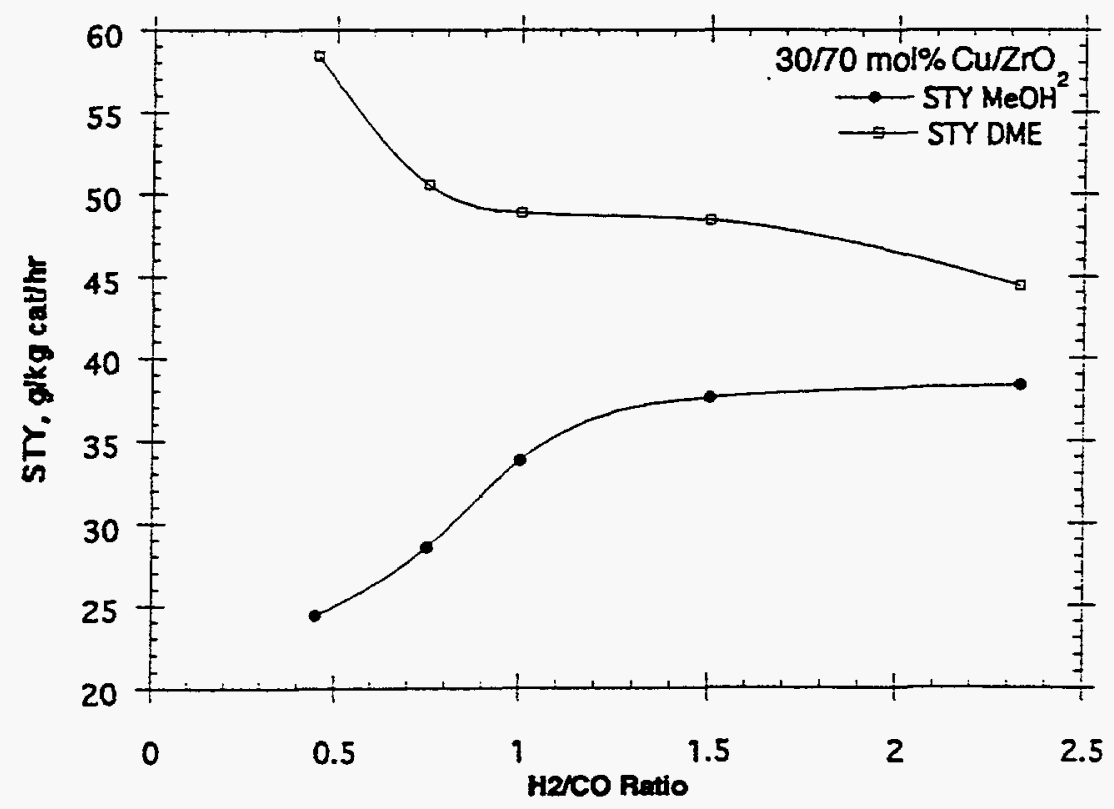


Since a significant effect on the catalytic behavior of the $\mathrm{Cu} / \mathrm{ZrO}_{2}$ catalysts was observed with addition of $\mathrm{CO}_{2}$ to the synthesis gas, the effect of the presence of water in the synthesis gas was investigated. A fresh portion of the $\mathrm{Cu} / \mathrm{ZrO}_{2} 10 / 90 \mathrm{~mol} \%$ catalyst was freshly calcined and reduced using the procedure described previously. The catalyst was then tested with $\mathrm{H}_{2} / \mathrm{CO}=$ 2.33 synthesis gas at $250^{\circ} \mathrm{C}, 7.6 \mathrm{MPa}$, and with $\mathrm{GHSV}=6120 \mathrm{l} / \mathrm{kg}$ cat $/ \mathrm{hr}$. Once steady-state conversion was attained, water was injected at the top of the preheater section of the reactor into the $\mathrm{H}_{2}$ /CO reactant mixture with a Gilson liquid pump. The inlet of the reactor was heated to $125-130^{\circ} \mathrm{C}$ to ensure that the water was vaporized as it entered the reactor. Water injection rates of $0.5,1.0,2.0,3.0,5.0$, and $6.0 \mathrm{ml} / \mathrm{min}$ were utilized, and this also increased the total GHSV slightly. For every injection experiment, the catalytic data were collected for at least $10 \mathrm{hr}$ in order to confirm the steady-state condition.

The main products observed were methanol and dimethylether. As expected, $\mathrm{CO}_{2}$ was also produced as a result of the water gas shift reaction (WGSR). Methanol productivity (STY) showed a gradual decrease from $\gg 25 \mathrm{~g} / \mathrm{kg}$ cat $/ \mathrm{hr}$ when no water was injected to $» 4 \mathrm{~g} / \mathrm{kg}$ cat $/ \mathrm{hr}$ for a water injection rate of $6.0 \mathrm{ml} / \mathrm{min}$, as indicated in Table 3.3.8 and Figure 3.3.11. Likewise, DME productivity also decreased upon water injection, and the DME STY was nearly zero for the higher water injection rates, as shown in Figure 3.3.11. In contrast, the formation of $\mathrm{CO}_{2}$ via the WGSR showed a continuous increase with the amount of water injected, wherein the $\mathrm{CO}_{2}$ STY increased from $30 \mathrm{~g} / \mathrm{kg}$ cat $/ \mathrm{hr}$ with no water injection to $131 \mathrm{~g} / \mathrm{kg}$ cat $/ \mathrm{hr}$ at the highest water injection rate of $6.0 \mathrm{ml} / \mathrm{min}$. Although the experimental procedures were different, it is interesting to note that the WGSR-generated $\mathrm{CO}_{2}$ did not promote methanol synthesis, as was the case when the $\mathrm{CO}$ reactant was progressively replaced by $\mathrm{CO}_{2}$.

Table 3.3.8 Water Injection Rate into the $\mathrm{H}_{2} / \mathrm{CO}=2.33$ Synthesis Gas Mixture and the Influence on the Reactant Composition, GHSV and Product Productivities at $250^{\circ} \mathrm{C}$ and $7.6 \mathrm{MPa}$

\begin{tabular}{|c|c|c|c|c|c|c||}
\hline $\begin{array}{c}\text { Water } \\
\text { Injection } \\
\text { Rate } \\
(\mathrm{m} / \mathrm{min})\end{array}$ & $\begin{array}{c}\mathrm{H}_{2} / \mathrm{CO} / \mathrm{H}_{2} \mathrm{O} \\
(\mathrm{mol} \%)\end{array}$ & $\begin{array}{c}\mathrm{GHSV} \\
(\mathrm{l} / \mathrm{kg} / \mathrm{hr})\end{array}$ & $\begin{array}{c}\mathrm{CO} \\
\text { Conv. } \\
(\mathrm{mol} \%)\end{array}$ & $\begin{array}{c}\mathrm{CH}_{3} \mathrm{OH} \\
\mathrm{STY} \\
(\mathrm{g} / \mathrm{kg} / \mathrm{hr})\end{array}$ & $\begin{array}{c}\mathrm{DME} \\
\mathrm{STY} \\
(\mathrm{g} / \mathrm{kg} / \mathrm{hr})\end{array}$ & $\begin{array}{c}\mathrm{CO}_{2} \\
\mathrm{STY} \\
(\mathrm{g} / \mathrm{kg} / \mathrm{hr})\end{array}$ \\
\hline 0.5 & $69.8 / 29.9 / 0.3$ & 6140 & 3.0 & 25.7 & 2.5 & 29.5 \\
\hline 1.0 & $69.5 / 29.8 / 0.7$ & 6160 & 4.3 & 22.6 & 1.3 & 49.5 \\
\hline 2.0 & $69.1 / 29.6 / 1.3$ & 6200 & 6.6 & 17.9 & $» 0$ & 83.9 \\
\hline 3.0 & $68.6 / 29.4 / 2.0$ & 6240 & 8.8 & 11.6 & $» 0$ & 107.0 \\
\hline 5.0 & $67.7 / 29.0 / 3.2$ & 6325 & 11.5 & 5.7 & $» 0$ & 123.6 \\
\hline 6.0 & $67.3 / 28.8 / 3.8$ & 6365 & 12.6 & 4.2 & $» 0$ & 131.0 \\
\hline
\end{tabular}


Figure 3.3.11 Effect of Water Injection on the Productivities, Expressed as Space Time Yields, of Methanol and Dimethylether from $\mathrm{H}_{2} / \mathrm{CO}=2.33$ Synthesis Gas at $250^{\circ} \mathrm{C}$ and

7.6 MPa with GHSV $=6120 \mathrm{l} / \mathrm{kg} \mathrm{cat} / \mathrm{hr}$

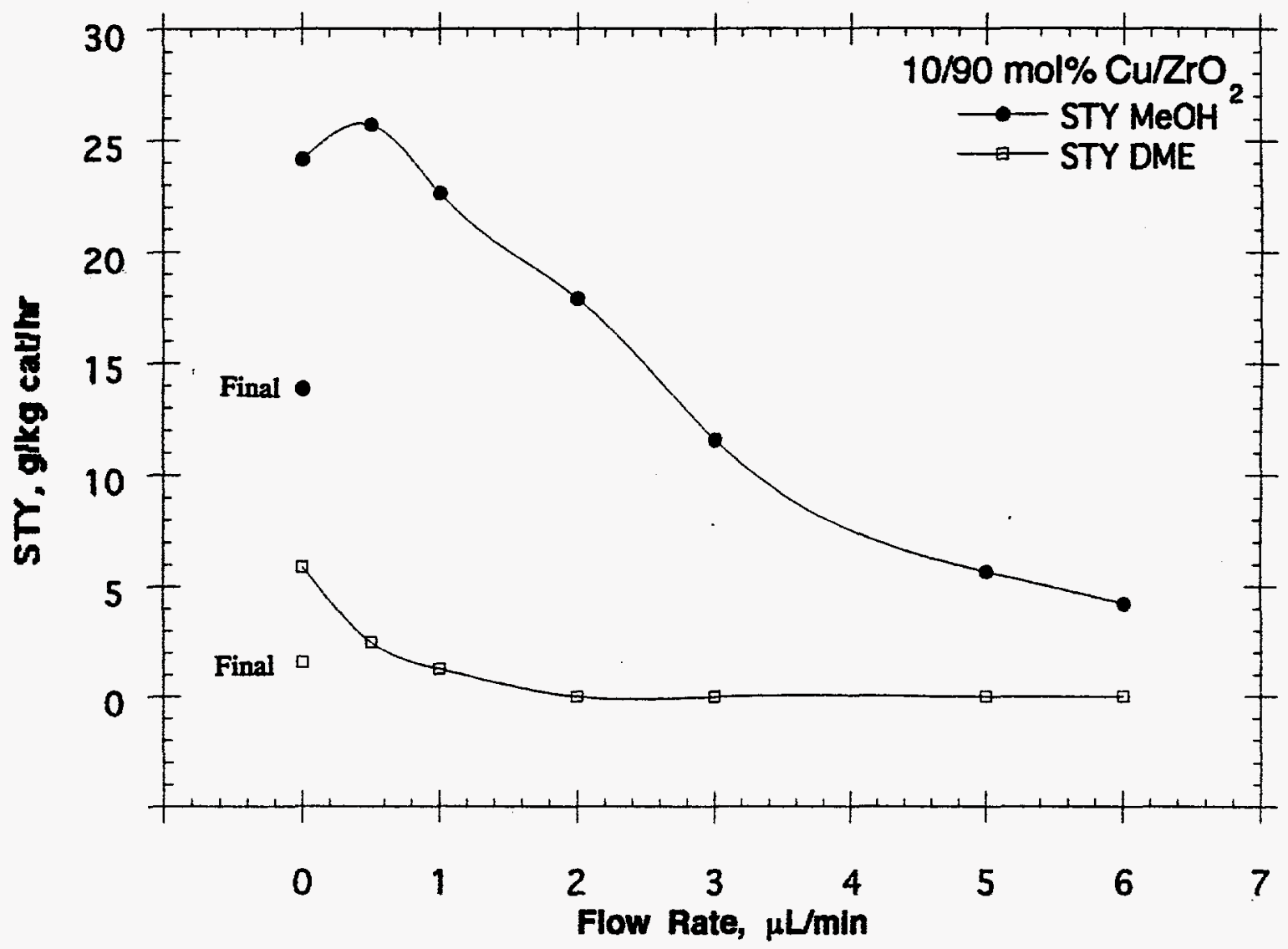

After completion of the water injection experiments, testing was carried out under the initial reference conditions to compare the final activity of the catalyst with the initial activity. The catalyst showed deactivation, and the methanol and DME productivities were 14 and $1.6 \mathrm{~g} / \mathrm{kg}$ $\mathrm{cat} / \mathrm{hr}$, respectively, as shown by the data points in Figure 3.3.11. Chemical analysis of the catalyst showed that no significant iron deposition via iron carbonyl occurred during the testing (i.e., it was below the detection limit of $<79 \mathrm{ppm}$ for the size of the sample submitted for analysis).

\section{Double Bed Catalyst Studies}

In a continuation of alcohol synthesis over $\mathrm{ZnO} / \mathrm{Cr}_{2} \mathrm{O}_{3}$-based catalysts, both alone and in tandem in the two catalyst bed configuration (see quarterly technical progress report 3QFY95), testing at higher reactor pressure was initiated using a high GHSV of $18,3751 / \mathrm{kg}$ cat $/ \mathrm{hr}$. In the double bed experiment, the top bed consisted of $3 \mathrm{~mol} \% \mathrm{Cs} / \mathrm{Cu} / \mathrm{ZnO} / \mathrm{Cr}_{2} \mathrm{O}_{3}$ while the lower bed was composed of a $4 \mathrm{~mol} \% \mathrm{Cs} / \mathrm{ZnO} / \mathrm{Cr}_{2} \mathrm{O}_{3}$ catalyst. Doping of both catalysts after calcination in $\mathrm{N}_{2}$ was carried out using a $\mathrm{N}_{2}$-purged aqueous solution of $\mathrm{CsOOCH}$ solution with a $\mathrm{CuO} / \mathrm{ZnO} / \mathrm{Cr}_{2} \mathrm{O}_{3}$ 
catalyst prepared via a hydrotalcite precursor and a commercial $\mathrm{ZnO} / \mathrm{Cr}_{2} \mathrm{O}_{3}$ catalyst (Harshaw $\mathrm{Zn}-0311 \mathrm{~T} 1 / 4^{\prime \prime}$ ). The two catalyst beds were reduced separately with a 2 vol\% $\mathrm{H}_{2} / \mathrm{N}_{2}$ gas mixture at $250^{\circ} \mathrm{C}$ (top bed) and $400^{\circ} \mathrm{C}$ (bottom bed). The inital testing of the catalyst was carried out at $7.6 \mathrm{MPa}$ with $\mathrm{H}_{2} / \mathrm{CO}=0.75$ synthesis gas with $\mathrm{GHSV}=18,3751 / \mathrm{kg} \mathrm{cat} / \mathrm{hr}$, and the temperatures of the top and bottom beds were maintained at 325 and $405^{\circ} \mathrm{C}$, respectively.

After steady-state testing under these conditions, the pressure of the reactor was increased to 10.5 $\mathrm{MPa}(1500 \mathrm{psig})$ and then to $12.6 \mathrm{MPa}(1800 \mathrm{psig})$. The experiment will be continued by reducing the reaction pressure back to $10.5 \mathrm{MPa}$ and then to $7.6 \mathrm{MPa}$. Both TCD and FID GC detectors were used for exit stream analysis, and condensible products were collected in a cold trap for subsequent GC/MS analyses. Confirmation of all reaction products is being carried out, and the results of this testing will be presented in the next quarterly report when the data analysis will be complete.

\section{Overall 1QFY96 Objectives}

Future plans for Task 3 will focus on the following areas:

(i) Continue studies of increasing the conversion of $\mathrm{H}_{2} / \mathrm{CO}$ to higher alcohols by promotion of the $\mathrm{C}_{1} \rightarrow \mathrm{C}_{2}$ carbon chain growth step over Cs-promoted $\mathrm{Cu} / \mathrm{ZnO} / \mathrm{Cr}_{2} \mathrm{O}_{3}$ and $\mathrm{MoS}_{2}$ catalysts,

(ii) Enhance the $\mathrm{C}_{2} \rightarrow \mathrm{C}_{3} \rightarrow \mathrm{C}_{4}$ carbon chain growth steps over $\mathrm{Cs} / \mathrm{Cu} / \mathrm{ZnO} / \mathrm{Cr}_{2} \mathrm{O}_{3}$ catalysts and $\mathrm{Cs} / \mathrm{Cu} / \mathrm{ZnO} / \mathrm{Cr}_{2} \mathrm{O}_{3} \| \mathrm{Cs} / \mathrm{ZnO} / \mathrm{Cr}_{2} \mathrm{O}_{3}$ dual bed catalysts, and

(iii) Prepare and test high surface area $\mathrm{Cu} / \mathrm{ZrO}_{2}$ catalysts, both Cs-doped and undoped, that are candidates for the synthesis of $\mathrm{C}_{1}-\mathrm{C}_{5}$ alcohols, in particular branched products such as isobutanol.

\subsubsection{Study of Reaction Conditions and Promotion of Group VIII Metals for Isobutanol Synthesis (University of Delaware)}

\section{Introduction}

Different precipitation procedures for mixed-oxide catalyst preparations are thought to be important for altering structures and compositions, since the precipitation sequence of each compound formed, its crystallinity and homogeneity, can be varied in this way. However, the effects of these variables are not very significant based on experimental observations. This is consistent with previous results on precipitation methods, from which it was observed that the catalysts made by the normal, reverse, and $\mathrm{pH}$-swing precipitation methods display basically the same catalytic behavior in higher alcohol synthesis. One likely explanation of the results is that the solid structures of the catalysts prepared by the different methods each transformed to the same final structure during calcination, reduction, and reaction. This notion is supported by the identical XRD patterns of the used catalysts in the previous study. These results also imply that the solid structure, especially the structure of the active state of the catalysts, is not thermally stable enough to withstand pretreatment and reaction conditions. To solve this problem, identification of the active structure or active phase (i.e., the structure and how to identify it) will 
be a crucial aspect of future study. Once this structure is established, we can be more specific on how to stabilize the site rather than the mixture as a whole and therefore be closer to our target.

The washing process plays a role in establishing the performance of these catalysts. Catalysts washed with water prove to have better isobutanol selectivity than either the unwashed or wateracetone washed catalysts. Although generally this might be attributed to differences in porosity or accessible surface area, in this case it seems more likely that residues on the surface poison or cover active sites. Furthermore, removal of excess alkali is probably most important, since alkali doping does not improve these catalysts, but rather tends to render them less active and selective.

Finally, an effort has been made to reduce the copper level of the best catalyst composition uncovered from earlier studies. However, no significant difference between the catalytic performance of the new low-copper catalysts and the earlier high-copper catalysts was observed. This was hinted at and can be explained in terms of the previous findings of the factorially designed catalyst preparation experiments.

\section{Experimental}

The catalysts discussed in this quarterly report, together with their compositions and preparation methods, are summarized in the Table 3.3.9.

Catalyst CF6KOHN was made at constant $\mathrm{pH}(=12)$ by adding both the mixed-nitrate and $\mathrm{KOH}$ solutions at the same time to the mixing tank (adjusting dropping rate to keep $\mathrm{pH}$ constant). It has the same composition as our best catalyst, F6KOHN (see Table 3.3.9). Three catalysts derived from this catalyst precursor were unwashed CF6KOHN (calcined without washing of the precipitate), washed CF6KOHN (calcined after thoroughly washing the precipitate), and washed, Li doped CF6KOHN (washed CF6KOHN, calcined and doped with $\mathrm{LiNO}_{3}$ ).

Catalyst 62895 was made by instant mixing of nitrates and $\mathrm{KOH}$ solutions. This catalyst had exactly the same composition as F6KOHN except that no copper was included.

Catalyst 71195 was prepared in order to examine the effects of higher, final precipitation $\mathrm{pH}$ and longer aging time on performance.

Catalysts made to evaluate the effects of the lowered copper level and the water or water-acetone washing process were $72095 \mathrm{~W}, 72095 \mathrm{~A}$ (F6KOHN made with water wash or water-acetone wash), 72195A (half the copper level of 72095A, acetone washed), 72195W (half the copper of level 72095W, water washed), and 72495A (1/4 copper level of 70295A, acetone washed).

The standard reaction test procedures used in this study were as follows: each catalyst was reduced at $260^{\circ} \mathrm{C}$ with $5 \% \mathrm{H}_{2} / \mathrm{N}_{2}$ for 24 hours, and tested at $350-425^{\circ} \mathrm{C}, \mathrm{CO} / \mathrm{H}_{2}=1,950$ psi and $\mathrm{GHSV}=2900 / \mathrm{h}$. 


\section{Table 3.3.9 Catalyst Compositions}

Catalyst

F6KOHN

unwashed Same as above

-CF6KOHN

CF6KOHN Same as above

CF6KOHNLi Same as above except $4 \mathrm{wt} \% \mathrm{LiNO}_{3}$ doping

71195

$72095 \mathrm{~W}$

72095A Same as above

72195W $\quad \mathrm{MnO} / \mathrm{CuO}=1, \mathrm{ZnO} / \mathrm{CuO}=1$,

72195A Same as above

$72495 \mathrm{~A}$
$\mathrm{MnO} / \mathrm{CuO}=0.5, \mathrm{ZnO} / \mathrm{CuO}=0.5$, $\mathrm{ZrO}_{2} / \mathrm{CuO}=2, \mathrm{CoO}=0.2 \mathrm{wt} \%$ (mole ratios) $\mathrm{ZrO}_{2} / \mathrm{CuO}=4, \mathrm{CoO}=0.2 \mathrm{wt} \%$ (mole ratios, $1 / 2 \mathrm{Cu}$ level of 72095)

Same as $\mathrm{F} 6 \mathrm{KOHN}, \mathrm{LiNO}_{3}=4 \mathrm{wt} \%$

$\mathrm{MnO} / \mathrm{CuO}=2, \mathrm{ZnO} / \mathrm{CuO}=2$, $\mathrm{ZrO}_{2} / \mathrm{CuO}=8, \mathrm{CoO}=0.2 \mathrm{wt} \%$

(mole ratios, 1/4 Cu level of 72095)
Preparation Method

Coprecipitation by dropping $\mathrm{KOH}$ to nitrate solution ( $\mathrm{pH}<0$ to 12 ), wash twice. Aging for 3 hours. Calcine at $400^{\circ} \mathrm{C} / \mathrm{N}_{2} / 3 \mathrm{~h}$

Constant $\mathrm{pH}=12$ coprecipitation, no washing triple times

Constant $\mathrm{pH}=12$ coprecipitation, no washing triple times

Same as above

Same as above except final $\mathrm{pH}=13.5$ and aging for 8 hours

Coprecipitation by dropping $\mathrm{KOH}$ to nitrate solution ( $\mathrm{pH}<0$ to 12 ). Aging for 3 hours. Washing three times with $\mathrm{H}_{2} \mathrm{O}$. Drying at $100^{\circ} \mathrm{C} / 18 \mathrm{~h}$. Calcine at $400^{\circ} \mathrm{C} / \mathrm{N}_{2} / 3 \mathrm{~h}$.

Same as above except that the precipitate was further washed with acetone before drying and calcination

Same as $72095 \mathrm{~W}$

Same as above except that the precipitate was further washed with acetone before drying and calcination

same as above 


\section{Results and Discussion}

\section{Effects of Catalyst Preparation Methods}

The constant $\mathrm{pH}$ precipitation method of preparation did not lead to any significant change in catalyst performance. CF6KOHN displayed very similar behavior to catalyst F6KOHN, which was reported on earlier. At a very early stage in this project, we also reported that different precipitation methods, namely, normal, reverse, and $\mathrm{pH}$ swing precipitations, showed little effect on catalytic performance. Based on that work, the activity of catalyst 62895 , made by instant mixing of nitrate and $\mathrm{KOH}$ solutions, was attributed to the absence of copper. The catalyst exhibited lower $\mathrm{CO}$ conversion and liquid yield, higher hydrocarbon selectivity, and lower isobutanol selectivity when compared with washed CF6KOHN. Therefore, it seemed necessary to include the copper component in the catalyst to achieve both high activity and isobutanol selectivity. To avoid copper agglomeration, maintain high activity and produce high isobutanol selectivity, lowering the copper content while simultaneously increasing its stability and dispersion would be a useful goal. Catalyst 71195 was prepared to examine the effects of higher, final precipitation $\mathrm{pH}$ and longer aging time on catalytic performance. Neither of these variables showed a significant effect on performance under our standard reaction test conditions. This can be seen from the very similar activity of 71195 and that of the previously studied F6KOHNLi.

\section{The Effects of Washing Procedures on Catalytic Performance}

From the results on the unwashed and washed CF6KOHN, it can be seen that washing of the precipitate or hydrogel plays an important role in achieving high activity and isobutanol selectivity. The washed CF6KOHN not only showed significantly higher $\mathrm{CO}$ conversion and liquid yield, but it also showed better isobutanol selectivity than did unwashed-CF6KOHN. This result may be accounted for if the washed CF6KOHN has a higher porosity and surface area or less residue covering the active sites. The washing step may also modify the surface composition of the catalyst prior to calcining. Removing excess alkali ions and other soluble components by washing the precipitate may result in better exposure of and access to the active sites on the surface.

Interestingly, lithium doping on the washed CF6KOHN did not improve the catalyst. Instead, it slightly reduced CO conversion, liquid productivity and isobutanol selectivity. This result is consistent with our previous report on alkali doping having a negative effect, but it is not what is expected based on the literature. This suggests that these catalysts operate by a different mechanism than the retro-aldol condensation proposed by Klier, but they are quite similar to the catalysts prepared by Keim. This result is logical since, given the catalyst's low surface area, the simple incipient-wetness alkali doping method may actually cover active sites, thereby acting as a textural poison and not as a promoter (see Table 3.3.10). Residual alkali, not removed after precipitation, would presumably function in the same fashion. 
Table 3.3.10 Performance of the Catalysts

\begin{tabular}{|c|c|c|c|c|c|c|c|c|c|}
\hline Catalysts & $\mathrm{T}^{\circ} \mathrm{C}$ & $\begin{array}{c}\mathrm{CO} \\
\text { conv.\% }\end{array}$ & $\begin{array}{c}\mathrm{CH} \\
\text { selec.\% }\end{array}$ & $\begin{array}{c}\mathrm{CO} 2 \\
\text { selec. } \%\end{array}$ & $\begin{array}{c}\mathrm{MeOH} \\
\mathrm{wt} \%\end{array}$ & $\begin{array}{c}\mathrm{nPrOH} \\
\mathrm{wt} \%\end{array}$ & $\begin{array}{c}\mathrm{iBuOH} \\
\mathrm{wt} \%\end{array}$ & $\begin{array}{c}\text { Other } \\
\text { wt\% }\end{array}$ & $\begin{array}{l}\text { Yield } \\
\mathrm{g} / \mathrm{g} / \mathrm{h}\end{array}$ \\
\hline Unwashed & 350 & 3 & 12 & 65 & 50.4 & 6.5 & 7.6 & 35.4 & 0.013 \\
\hline \multirow[t]{2}{*}{ CF6KOHN } & 400 & 8 & 14 & 55 & 30.0 & 9.5 & 4.8 & 55.7 & 0.039 \\
\hline & 425 & 11 & 18 & 60 & 17.4 & 10.3 & 7.8 & 64.4 & 0.050 \\
\hline \multirow[t]{3}{*}{ CF6KOHN } & 350 & 12 & 7 & 30 & 69.0 & 1.9 & 15.7 & 13.4 & 0.050 \\
\hline & 400 & 22 & 12 & 37 & 25.9 & 3.1 & 24.2 & 46.7 & 0.080 \\
\hline & 425 & 27 & 22 & 42 & 15.9 & 9.3 & 15.6 & 59.1 & 0.085 \\
\hline \multirow[t]{3}{*}{ CF6KOHNLi } & 350 & 5 & 14 & 52 & 66.8 & 3.3 & 14.8 & 15.0 & 0.038 \\
\hline & 400 & 17 & 19 & 47 & 29.6 & 10.5 & 17.6 & 42.3 & 0.078 \\
\hline & 425 & 22 & 26 & 46 & 19.6 & 13.8 & 11.0 & 55.5 & 0.067 \\
\hline \multirow[t]{3}{*}{62895} & 350 & 7 & 9 & 30 & 85.0 & & 10.9 & 4.1 & 0.033 \\
\hline & 400 & 12 & 20 & 44 & 44.4 & 3.3 & 10.0 & 42.4 & 0.038 \\
\hline & 425 & 18 & 32 & 47 & 16.8 & 5.1 & 10.0 & 68.1 & 0.050 \\
\hline \multirow[t]{3}{*}{71195} & 350 & 8.7 & 8.9 & 29.9 & 76.8 & 2.7 & 16.5 & 4.0 & 0.045 \\
\hline & 400 & 22.9 & 16.3 & 39.5 & 34.9 & 9.5 & 21.6 & 34.1 & 0.076 \\
\hline & 425 & 27.6 & 26.1 & 46.8 & 22.1 & 11.3 & 12.8 & 53.8 & 0.078 \\
\hline
\end{tabular}

In conjunction with this result, we note that the washing process did show an effect on the liquid product distributions of the catalysts. Comparisons of 72095A, 72095W, 72195A, and 72195W indicate that the water-only washing procedure is better than the water-acetone procedure for enhancing the isobutanol selectivity and productivity, as shown in Table 3.3.11. This implies that water, with its high solubility, more effectively removes excess alkali and other solubles that may inhibit activity. 
Table 3.3.11 Liquid Distributions (wt \%) and Yields (g/g/h)

\begin{tabular}{|c|c|c|c|c|c|c|}
\hline & $\mathrm{MeOH}$ & EtOH & $\mathrm{nPrOH}$ & $\mathrm{iBuOH}$ & Other & Yield \\
\hline \multicolumn{7}{|l|}{$72095 \mathrm{~A}$} \\
\hline $350^{\circ} \mathrm{C}$ & 81.5 & 0.0 & 1.1 & 14.3 & 3.1 & 0.064 \\
\hline $400^{\circ} \mathrm{C}$ & 31.2 & 0.7 & 1.0 & 20.4 & 46.6 & 0.070 \\
\hline $425^{\circ} \mathrm{C}$ & 20.0 & 0.6 & 1.6 & 18.7 & 59.0 & 0.052 \\
\hline \multicolumn{7}{|l|}{$72095 \mathrm{~W}$} \\
\hline $350^{\circ} \mathrm{C}$ & 74.1 & 0.0 & 1.0 & 16.1 & 8.9 & 0.060 \\
\hline $400^{\circ} \mathrm{C}$ & 37.1 & 0.0 & 1.3 & 26.6 & 35.0 & 0.073 \\
\hline $425^{\circ} \mathrm{C}$ & 21.3 & 0.4 & 1.6 & 21.8 & 54.9 & 0.060 \\
\hline \multicolumn{7}{|l|}{$72195 \mathrm{~A}$} \\
\hline $350^{\circ} \mathrm{C}$ & 69.2 & 0.6 & 0.7 & 12.7 & 16.9 & 0.075 \\
\hline $400^{\circ} \mathrm{C}$ & 28.7 & 0.0 & 0.8 & 17.7 & 52.8 & 0.059 \\
\hline $425^{\circ} \mathrm{C}$ & 15.1 & 0.3 & 1.0 & 16.8 & 66.8 & 0.061 \\
\hline \multicolumn{7}{|l|}{$72195 W$} \\
\hline $350^{\circ} \mathrm{C}$ & 72.20 & 0.0 & 0.7 & 14.7 & 12.7 & 0.064 \\
\hline $400^{\circ} \mathrm{C}$ & 33.2 & 0.5 & 0.9 & 25.9 & 39.5 & 0.073 \\
\hline $425^{\circ} \mathrm{C}$ & 16.0 & 0.7 & 1.1 & 18.4 & 63.7 & 0.059 \\
\hline \multicolumn{7}{|l|}{$72495 \mathrm{~A}$} \\
\hline $350^{\circ} \mathrm{C}$ & 71.1 & 0.0 & 0.4 & 11.1 & 17.3 & 0.073 \\
\hline $400^{\circ} \mathrm{C}$ & 28.2 & 0.6 & 0.9 & 20.7 & 49.6 & 0.061 \\
\hline $425^{\circ} \mathrm{C}$ & 21.1 & 1.1 & 0.9 & 18.7 & 58.2 & 0.059 \\
\hline
\end{tabular}

Reaction conditions: $950 \mathrm{psi}, 2900 \mathrm{~h}^{-1}$ 
The water-acetone washing procedure did not improve the surface area of the catalysts; (see $70295 \mathrm{~A}$ vs. $72095 \mathrm{~W}, 72195 \mathrm{~A}$ vs. $72195 \mathrm{~W}$ in Table 3.3.12) taken together with the fact that the procedure worsened isobutanol selectivity, it is clear that any anticipated benefit of the acetonewater washing procedure on physical properties, and, hence, selectivity is nonexistent. The results, on the other hand, do indicate that different washing procedures can have an impact on the performance of the catalysts, probably through removal or lack of removal of excess base. The observation that more than $50 \%$ of the surface area of the catalysts collapses after prolonged on-stream use indicates that the most active structures of the catalysts were probably destroyed in the early stages of treatment and reaction.

Table 3.3.12 Surface Area of the Catalysts $\left(\mathrm{m}^{2} / \mathrm{g}\right)$

\begin{tabular}{lcc} 
& Fresh & Used \\
\hline $720925 \mathrm{~A}$ & 108.2 & 47.5
\end{tabular}

$72095 \mathrm{~W}$

107.9

49.3

72195A

114.8

50.1

72195W

128.1

55.8

$72495 \mathrm{~A}$

116.9

56.9

\section{Effects of Copper Level of the Catalysts}

Based on previous results and discussions at the Annual Review in Pittsburgh, the copper level of the catalysts was an important factor to be examined. Initially, one might have expected major modifications in catalyst performance with a decrease in copper levels, since we had seen from previous experiments that a catalyst without copper showed poor performance. However, in this set of experiments, when the copper level was decreased by half in 72195A and by one fourth in 72495A from the copper level of 72095A, no significant changes in catalyst activity, selectivity, and productivity were noted (see Tables 3.3.11 and 3.3.13). 
Table 3.3.13 Performance of the Catalysts (@ 950 psi, 2900 h$^{-1}$ )

$\mathrm{CO}_{2}$ Selectivity CxHy Selectivity CO Conversion $\mathrm{H}_{2}$ Conversion (\%)

(\%)

(\%)

(\%)

$72095 \mathrm{~A}$

$350^{\circ} \mathrm{C}$

25.5

8.3

11.4

12.6

$400^{\circ} \mathrm{C}$

38.0

17.2

23.2

18.3

$425^{\circ} \mathrm{C}$

44.5

28.4

26.0

21.7

72095W

$$
350^{\circ} \mathrm{C}
$$

21.0

6.1

13.5

13.4

$400^{\circ} \mathrm{C}$

34.2

14.9

25.3

18.5

$425^{\circ} \mathrm{C}$

36.5

24.9

30.1

22.9

72195A

$350^{\circ} \mathrm{C}$

26.3

8.5

12.7

14.4

$400^{\circ} \mathrm{C}$

42.1

21.6

24.1

18.7

$425^{\circ} \mathrm{C}$

46.7

33.7

28.2

22.8

72195W

$$
350^{\circ} \mathrm{C}
$$

33.37

11.4

10.0

12.2

$400^{\circ} \mathrm{C}$

46.8

24.0

21.5

18.1

$425^{\circ} \mathrm{C}$

42.6

33.9

31.5

26.5

72495A

$350^{\circ} \mathrm{C}$

34.0

11.9

11.3

14.0

$400^{\circ} \mathrm{C}$

43.1

22.1

22.8

18.4

$425^{\circ} \mathrm{C}$

44.4

31.2

26.3

21.6 
Another interesting result observed was that surface area increased slightly with the decrease in copper level for both the fresh and used catalysts (see Table 3.3.12).

Taken together and with earlier work, these results indicate that the copper contents of catalysts can be significantly lowered. This could be important for preventing copper agglomeration. The decrease in the catalysts' copper content also increases the other metal oxide/CuO ratios of the catalysts. According to our factorially designed experiment, the metal oxide/CuO ratios play an important role in alcohol selectivities. Yet, we did not observed any significant changes in alcohol selectivities with the increase in oxide/CuO ratios for this set of catalysts. Two aspects need to be considered for rationalizing the two sets of results: (1) while $\mathrm{MnO} / \mathrm{CuO}, \mathrm{ZnO} / \mathrm{CuO}$, and $\mathrm{ZrO}_{2} / \mathrm{CuO}$ ratios increased in the order of $72095 \mathrm{~A} \rightarrow 72195 \rightarrow 72495$, the ratios among $\mathrm{MnO}, \mathrm{ZnO}$, and $\mathrm{ZrO}_{2}$ remained unchanged. The beneficial effect of increased $\mathrm{ZrO}_{2} / \mathrm{CuO}$ ratio on isobutanol selectivity might be offset by the negative effect of the increased $\mathrm{MnO} / \mathrm{CuO}$ ratio on isobutanol selectivity. Thus the factorially designed experiments left open the question of the effect of absolute $\mathrm{CuO}$ level. Presumably, if we had probed a range of copper levels between zero and $\sim 10 \mathrm{wt} \%$, the level in $72495 \mathrm{~A}$, then a significant effect would have been noted. Instead our experiments were conducted at copper levels above this critical content, and the effect of variation in copper seems minimal. (2) As seen from previous reports, most of our catalysts have behaved similarly, giving high $\mathrm{CO}_{2}$ selectivity, moderate $\mathrm{CO}$ conversion and hydrocarbon selectivity, moderate liquid productivity, and consistent liquid product distribution patterns. This might imply that our catalysts may be working close to equilibrium, that is., at reaction conditions (low pressure and relatively high reaction temperature) where reactions progress to their thermodynamic limits and are not under kinetic control. Therefore, further work needs to be done to optimize the reaction conditions while continuing the exploration of catalysts for enhanced isobutanol production. Also, thermodynamic computations will be done to examine this possible equilibrium effect.

\section{Conclusions}

The different precipitation methods of the mixed oxide catalysts discussed in this report do not show any significant effects on catalyst performance. However, thorough washing of the precipitates with water was proven to be a better procedure compared with the other methods tested. This agrees well with our earlier observation that the excess alkali doping does not improve these catalysts; thus pointing away from the retro-aldol condensation mechanism. Decreasing the copper level of the catalysts did not significantly change catalytic performance. This implies that the levels of the other components, such as $\mathrm{MnO}$ and $\mathrm{ZnO}$, need to be adjusted.

\subsection{Poison Resistant Catalysts Development and Testing}

The initial effort under this task is to build a mobile facility that will test feed gas and various feed gas cleanup methods at customer locations, as well as at Air Products. A secondary goal is to provide a laboratory that can be used for testing poison resistant catalysts, if such a program is warranted. The need for such a program will be evaluated after the costs of sulfur removal are analyzed. This latter effort is part of Bechtel's tasks, and it will be reported on separately by Bechtel. This report describes the design of the AF ${ }^{2}$ DU (Alternate Fuels Field Development Unit). 


\subsubsection{Design}

Schematic drawings of the $\mathrm{AF}^{2} \mathrm{DU}$ are presented in Figures 3.5.1-3.5.8. Since the system must be movable to a customer's location, it is mounted on a trailer, depicted in Figure 3.5.1. The trailer comprises laboratory and office areas, both of which will have adequate ventilation and alarm systems to protect occupants. Safety will be covered in Air Products' normal hazards review procedure. The trailer will meet all relevant OSHA and BOCA codes. This aspect along with a complete set of schematics and operating plans will be documented after the hazard review is completed.

The air conditioned and heated office area (Figure 3.5.2) contains an Allen-Bradley control system modeled after the Air Products standard system used in remote locations and not our laboratory systems. Its main advantage over Windows-based PC systems is that its robustness enables proper operation and data saving after a power failure. It can, of course, communicate electronically with Air Products' normal PC-based data analysis routines. Electronic data transmission over phone lines is provided for; two phone lines have been installed so that data can be transmitted as necessary. The office area also contains a desk, as well as work benches and chemical cabinets necessary for sample preparation and operation of the reactor. Under normal operating conditions, the entire $\mathrm{AF}^{2} \mathrm{DU}$ will be operated by a single person covering a 24 hour period.

The gas feed system, shown in Figure 3.5.3, allows the feed of selected poisons as well as syngas to the reactor system. Poison removal techniques can be tested in the adsorber system shown in Figure 3.5.4. The section contains five beds that can be filled with various materials. The beds can be regenerated automatically with temperature programming provided by the automatic control system. This system is also capable of blending feed gases from a second source. This feature could be used in testing at a location having recycle gas flow as well as in blending selected poisons into the feed.

The reactor system shown in Figure 3.5 .5 contains a $300 \mathrm{cc}$ reactor. The system is essentially a duplicate of that in the laboratory so that results between the field and the laboratory will be directly comparable.

The analytical system is shown in Figures 3.5.5-3.5.8. Since we will be testing for unknown materials as well as known poisons, the system is extensive and flexible. The actual analysis will be done in two Hewlet-Packard 6890 gas chromatographs. These are modern systems fitted with a variety of detectors that enable flexible analysis. The capability of the detectors is summarized in Table 3.5.1. Details of the system are given below. 


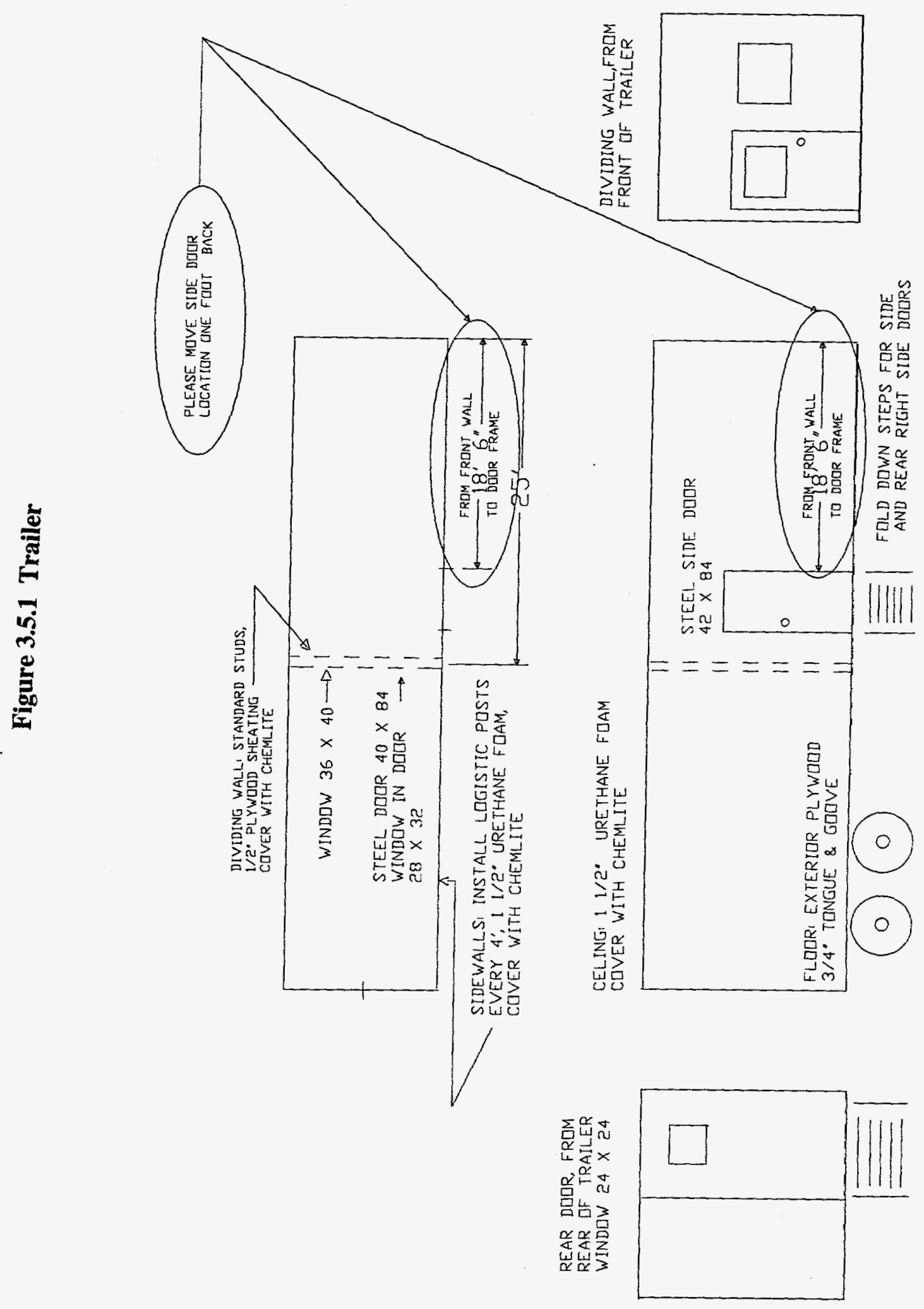

$N$ 


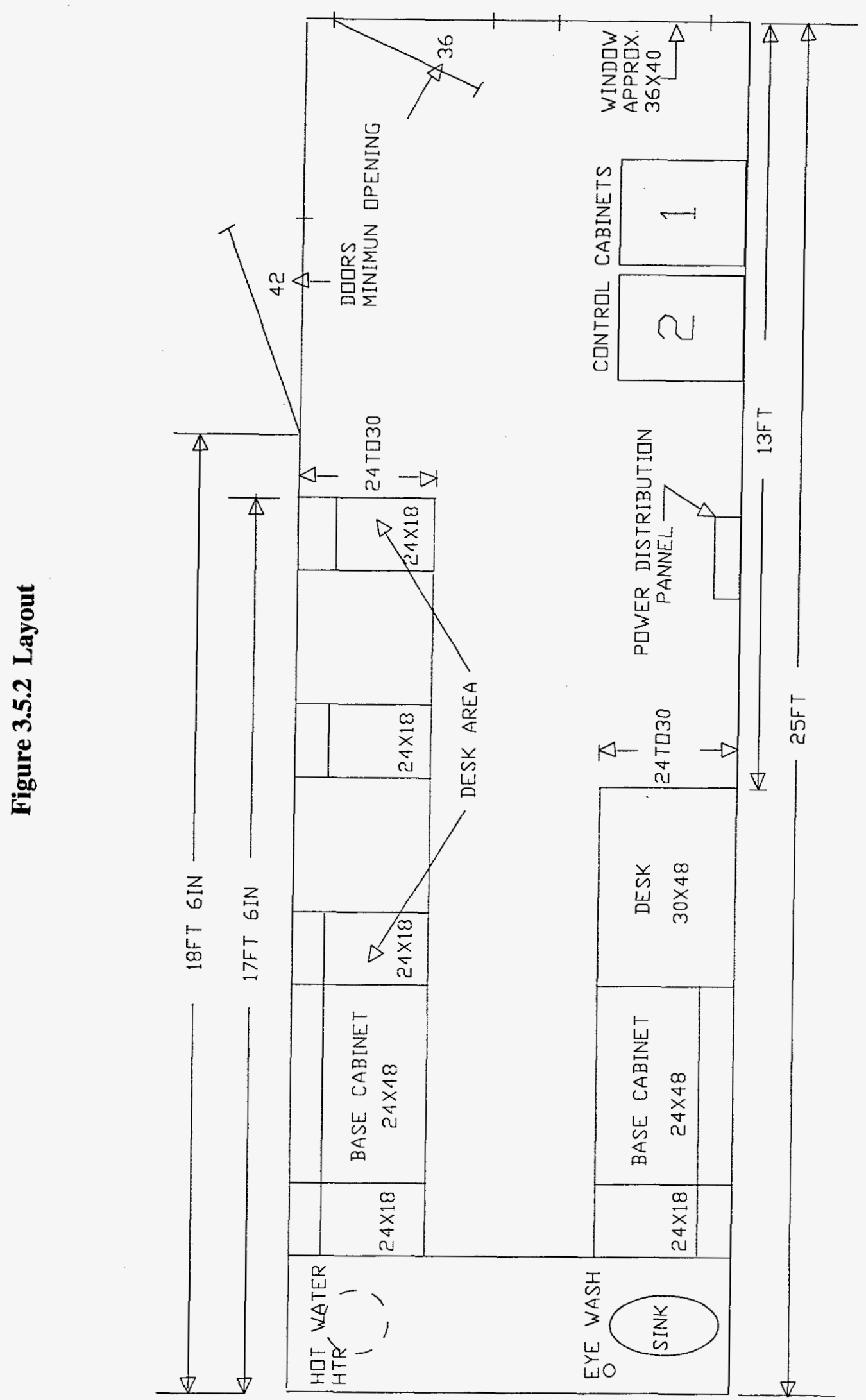




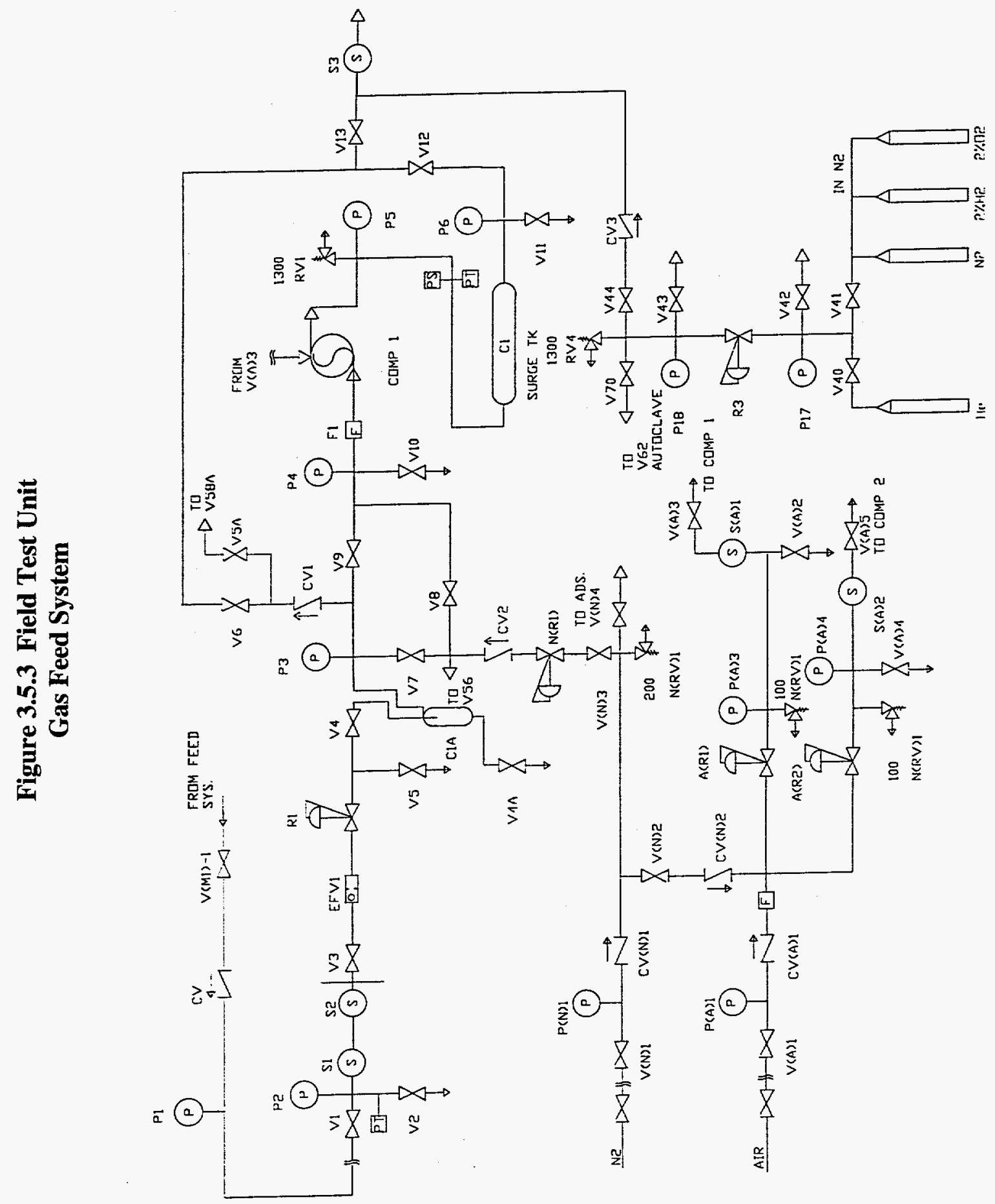



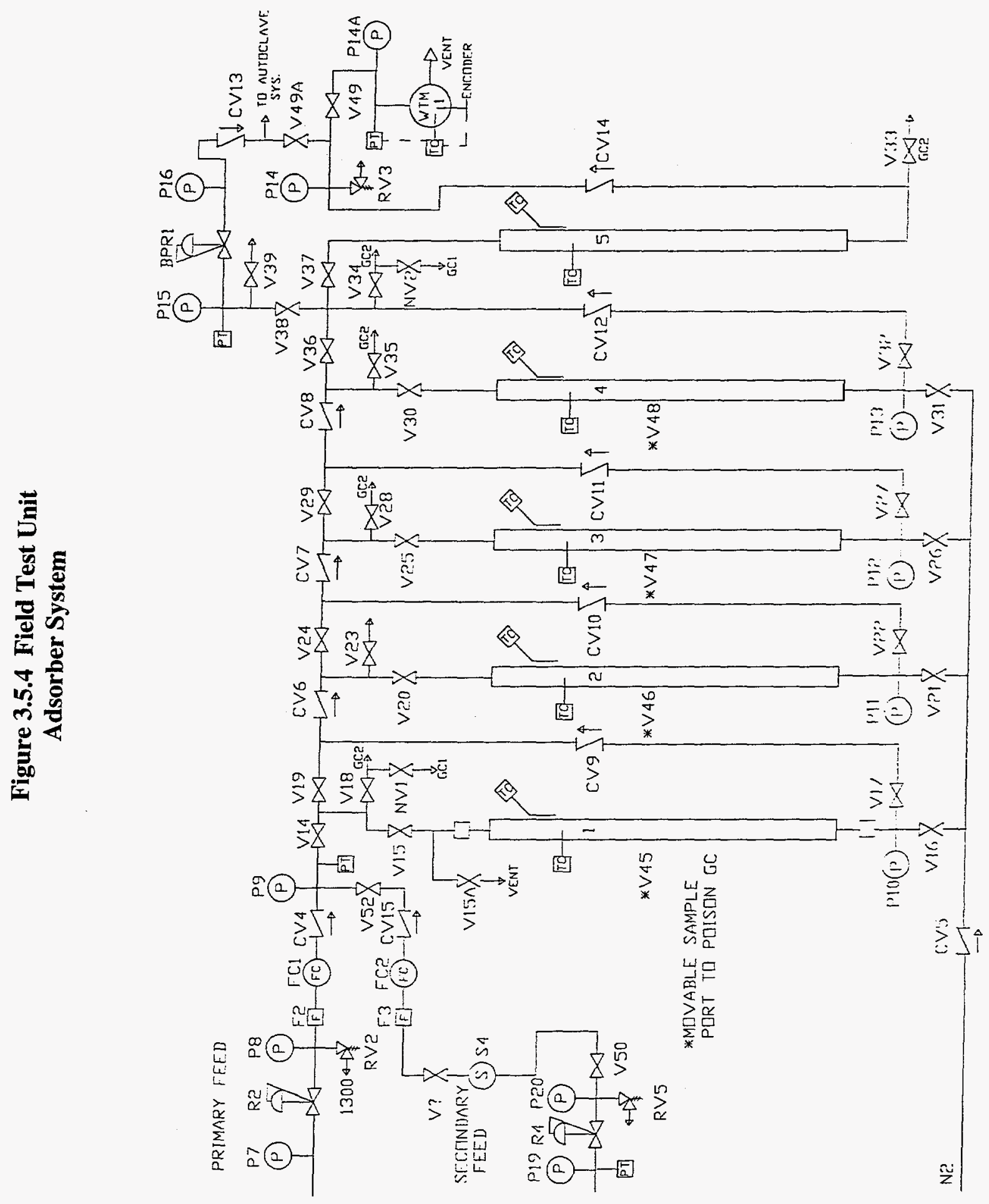

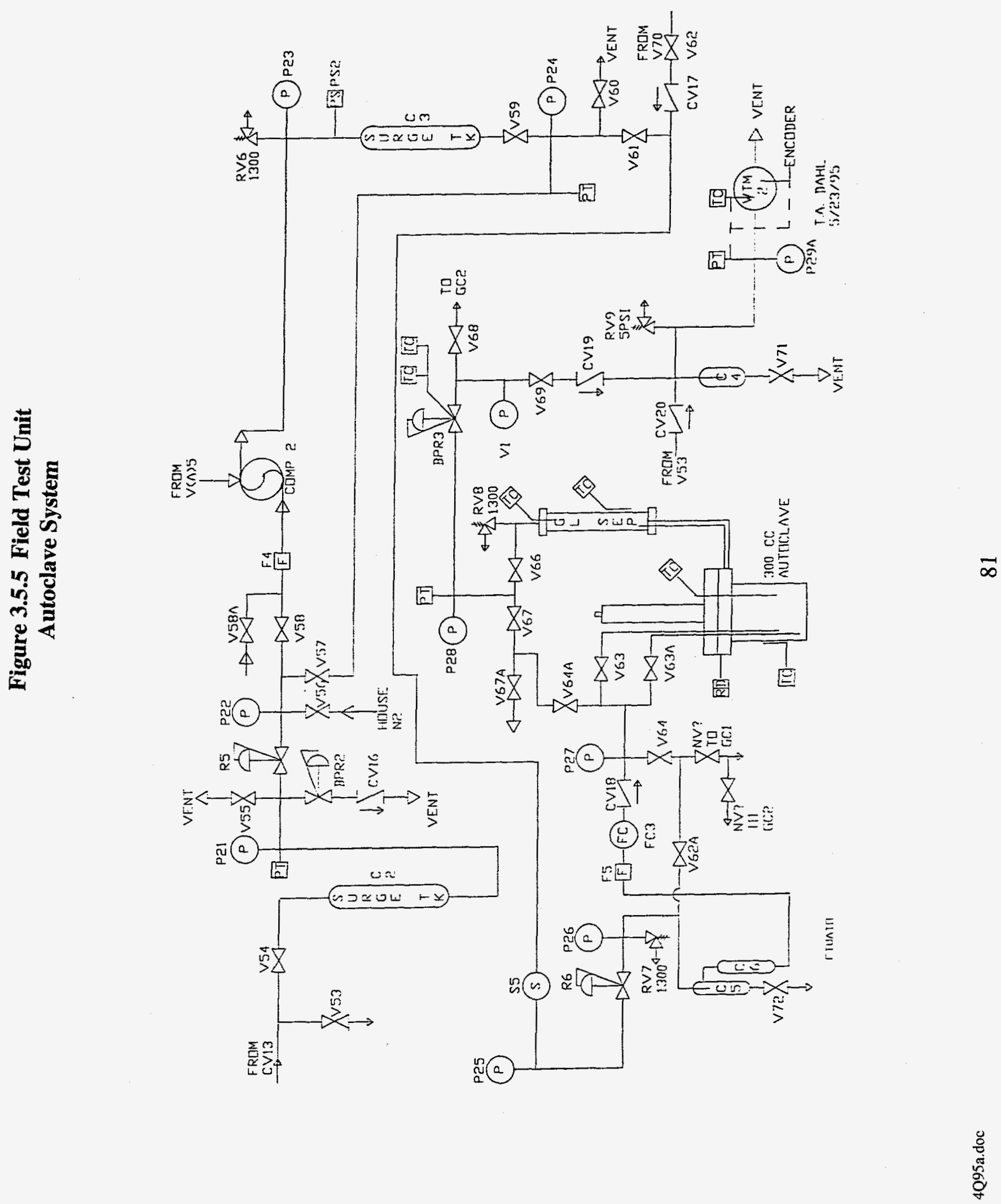

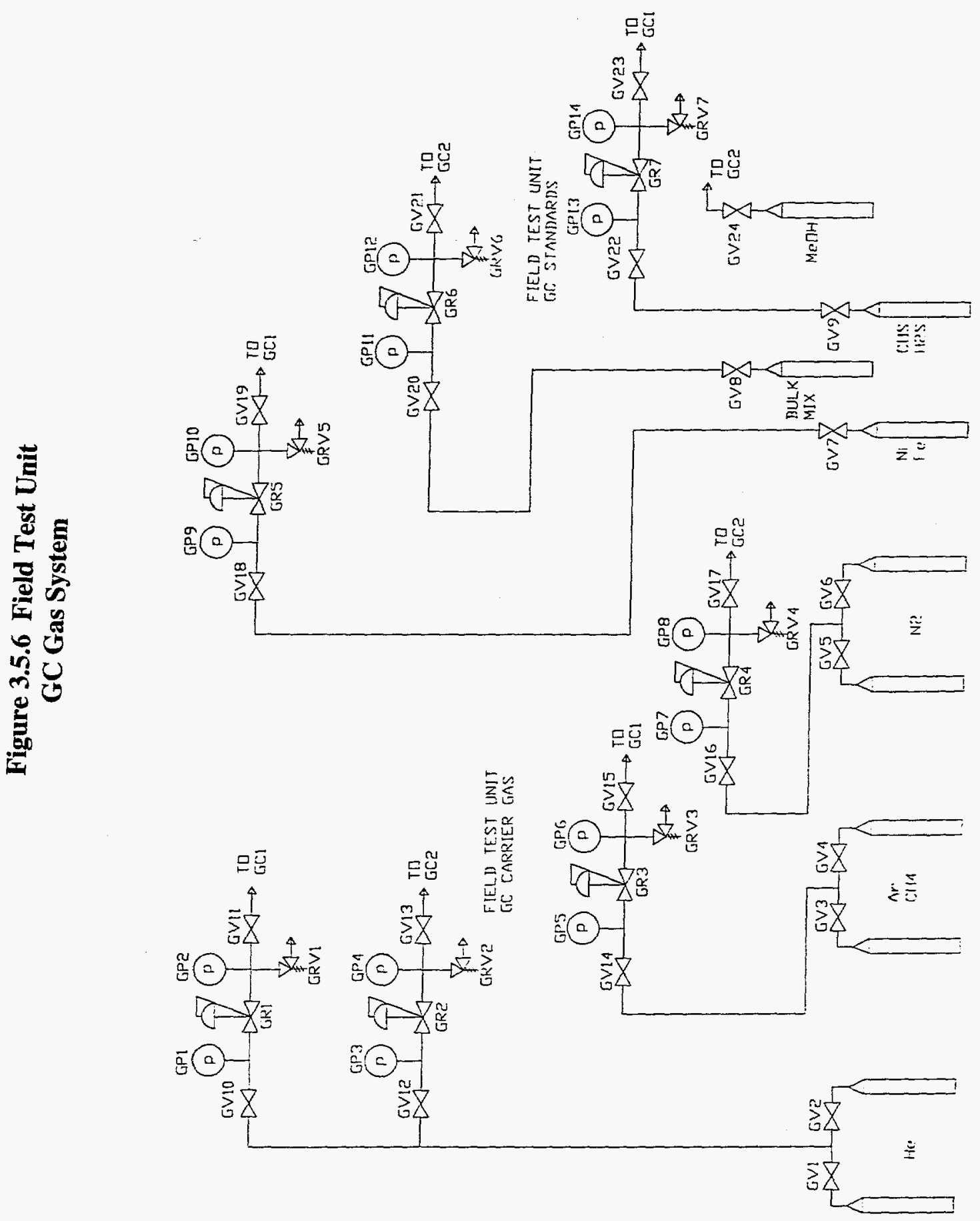

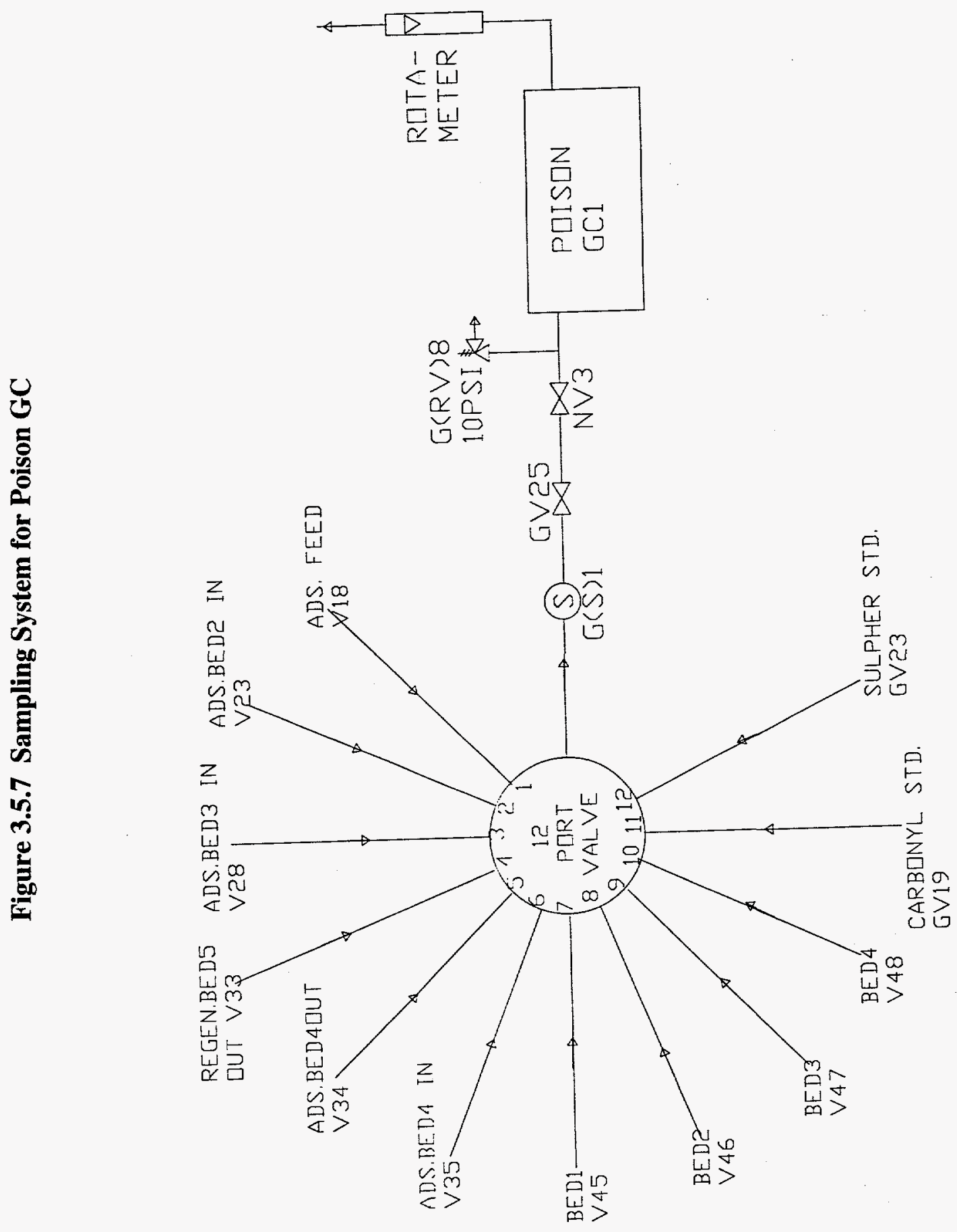


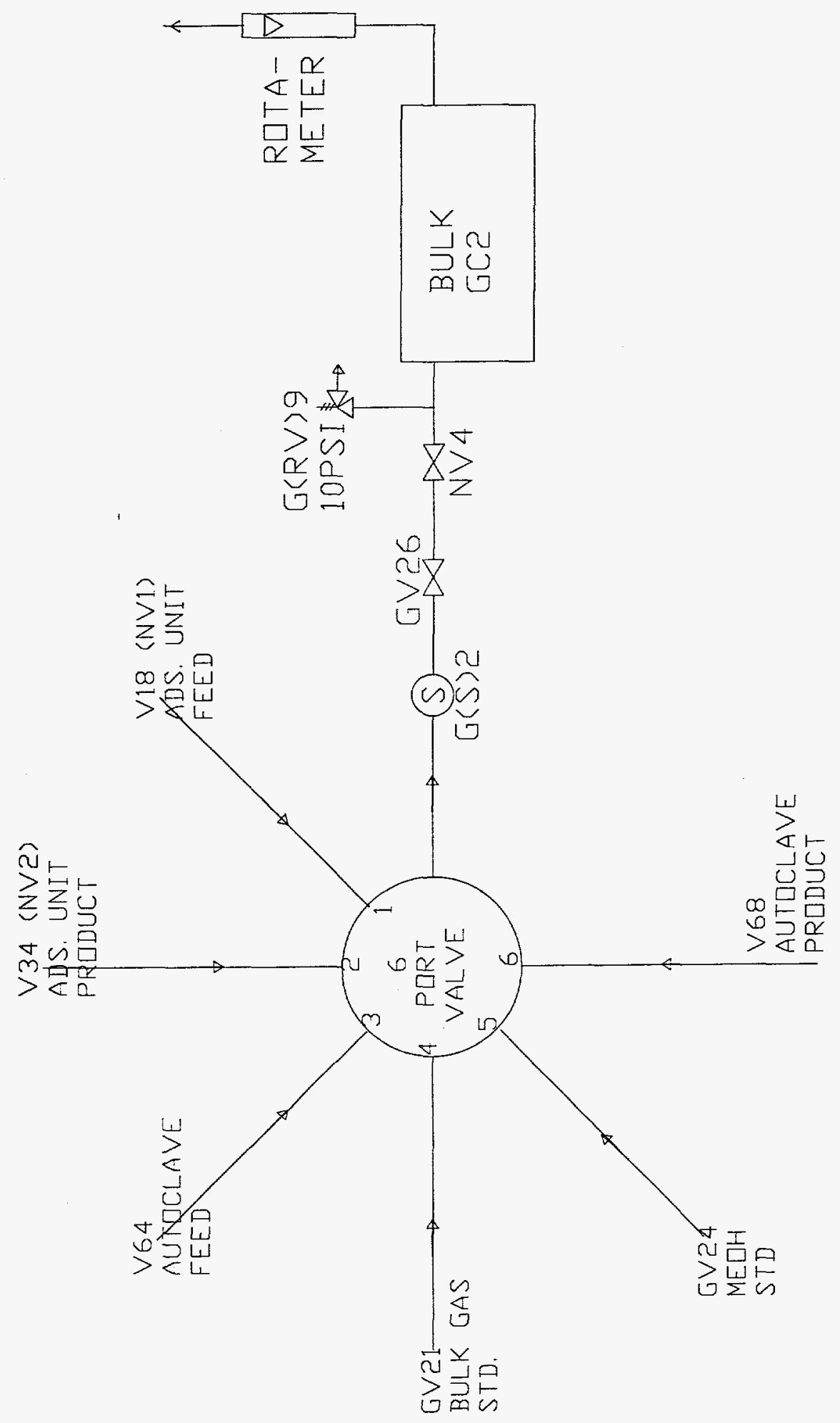

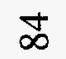

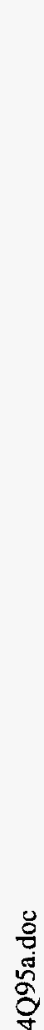




\section{Table 3.5.1 GC Detector Capabilities}

\author{
Detector \\ $\mathrm{TCD}$ (thermal conductivity) \\ FID(flame ionization) \\ $\mathrm{SCD}$ (sulfur chemiluminescence) \\ ECD(electron capture)
}

\author{
Substance(s) Measured \\ $\mathrm{CO}, \mathrm{CO}_{2}, \mathrm{~N}_{2}, \mathrm{H}_{2}$ \\ Organics(alcohols, etc.) \\ $\mathrm{S}$ \\ $\mathrm{Ni}, \mathrm{Fe}$
}

The "Poisons GC" will be an HP-6890 GC with one electronic pressure controlled (EPC) packed injector and the appropriate valving to do simultaneous injections of the sample into two columns. One column will be interfaced to an Hewlett-Packard Electron Capture Detector (ECD) to analyze nickel and iron carbonyls, and the other column will be interfaced to a Sievers Sulfur Chemiluminescence Detector (SCD) to conduct trace sulfur analysis. The SCD will monitor carbonyl sulfide, hydrogen sulfide and sulfur dioxide to $0.03 \mathrm{mg} / \mathrm{L}$.

The second "Bulk Gas GC" will be another HP-6890 GC with two thermal conductivity detectors (TCDs) and one flame ionization detector (FID) with full EPC. The FID will be configured to do hydrocarbon or alcohols analysis. The first TCD analyzes for carbon dioxide, oxygen, nitrogen, methane and carbon monoxide. The lower limit for all components is $200 \mathrm{ppm}$ except for carbon monoxide, which is $400 \mathrm{ppm}$. The second TCD detects hydrogen to a lower detection limit of $100 \mathrm{ppm}$. The TCD signals are electronically summed to provide a single signal output.

These GCs will be configured by Wasson ECE, a certified Hewlett-Packard channel supplier. The base HP-6890 instrument will be purchased from Hewlett-Packard and shipped to Wasson along with the Sievers SCD. Wasson will install the Sievers detector, the necessary valves and columns to perform the analyses stated above. Upon completing the configuration, Wasson will test and install the equipment, train personnel in the use of the equipment and guarantee the performance of the GCs to meet all the detection limits specified above. The Nelson Turbochrom equipment and computer work station will have to be ordered separately and installed in the trailer when the GCs arrive. Because Turbochrom cannot as yet fully control the new HP-6890 GC, Turbochrom 900 series A/D interfaces will have to be used.

The stream selection system will be automated by Chatham Instruments. Since integrators will not be used, the Nelson Turbochrom system will have to be able to control the stream select valves. This will require that some external computer code be written for Turbochrom to fully control and read the sample stream number from the stream select valves into the computer. The old stream select valves will be refurbished and used again in the new system to help control cost.

Careful selection of the detector, sampling ports and columns allows the capability of measuring poisons with only two Gcs, which is the number necessary to do normal laboratory analysis of reaction and feed products. 


\subsubsection{Schedule}

Since they are long lead time items, the trailer and the analytical systems were ordered in May 1995. A summary of the detailed schedule is provided in Table 3.5.2.

Construction of the trailer will be completed by the end of April when the shakedown run is completed. The project is on time and within budget for this quarter. The trailer has been purchased and the interior modified. The electrical hookups to the trailer have been completed, and utility hookup is underway.

\section{TASK 4: PROGRAM SUPPORT}

Bechtel's project personnel attended the DOE Contractors' Review Meeting on 28 through 31 August and a kick-off meeting at Air Products' Allentown office on 1 and 2 August. They reported the following:

- Task 1.3, Fischer-Tropsch Support (Catalyst/Wax Separation) - Due to reassignment of personnel, no work was performed on this task during the reporting period.

- Task 4.2, Commercial Applications (Mixed Alcohol Synthesis) - Additional modifications to the economic analysis spreadsheet models for the two scenarios of gasification coupled with mixed alcohol synthesis have been implemented, and the models are being tested.

Development of a model for the third scenario of a stand-alone mixed alcohol synthesis plant was started. Work on the second scenario, a refinery plant, was continued with the use of a linear programming model to analyze product slates and revenue.

- Task 4.5, Syngas Generation and Cleanup - Detailed material balances for the coal and coke feedstocks to predict the expected composition of the cleaned syngas discharging from several sulfur removal processes were developed and submitted to gasification vendors and acid gas removal equipment vendors for their comments. Various designs of a natural gas, partial oxidation gasifier to produce syngas were evaluated and, for two of these, estimates of capital and operating costs were made.

\section{TASK 5: PROJECT MANAGEMENT}

\subsection{Reports and Presentations}

A two-day meeting was held with DOE and subcontractors to kick off the Alternative-Fuels II and Slurry Bubble Column Reactor (Hydrodynamics) programs. Preliminary results from the June hydrodynamic run were presented at the meeting. Interesting discussions followed the presentations. DOE was quite impressed with the large number of objectives achieved in a single campaign.

A final topical report on the results of the Fischer-Tropsch II demonstration was completed. Comments and suggestions received from DOE personnel on the draft report were incorporated into the final report. 


\subsection{Management Activities}

A meeting was held with Reema International (now REMTECH) and DOE on August 29 to discuss Reema's interest in demonstrating their F-T process at LaPorte. Reema is a small, privately owned oil and gas company based in Denver, Colorado. Significant business and technical discussions are required before the project can be kicked off. An operating window of April-June 1996 was given, assuming we do not need significant modifications and the project can be kicked off in the October - November time frame.

\section{Table 3.5.2 Schedule Summary Field Test Unit \\ Revised August 221995}

Month

May :

Purchase Trailer

Power To Trailer

Order Analytical Equipment

Jun./Jul./Aug.:

Prepare Trailer

In Place At Iron Run

Floor, Walls, Ceiling,

Outside Door

Rear Door

Office Equipment

Work Bench /Storage Cabinets

Determine Lab Automation

Order Automation Components

Electrical

Transformer in Place

Distribution Panel

Heating

Lighting
Completion Date Vendor

Complete

Complete

Complete

Complete

Complete

Complete

Complete

Complete

Complete

1 Sept. 95

Dahl

Complete

Complete

1 Sept. 95

1 Sept. 95

Fac.Svcs.

Fac.Sves.

Sept./Oct./Nov.:

Utilities

Ventilation

Cooling

Phone Lines

15 Dec. 95

Air/Nitrogen

Syn. Gas Feed Lines
25 Aug. 95

25 Aug. 95

15 Sept. 95

HT Lyons.

15 Dec. 95

15 Dec. 95
HT Lyons.

HT Lyons.

Fac.Svcs. Water

HT Lyons.

HT Lyons 
Table 3.5.2 Schedule Summary (continued)

Month

Completion Date Vendor

Dec./Jan./Feb.96:

Install Lab Equipment

Allen Bradley Programming 15 Dec. 95 MIS

Excel Spread Sheet/PE Nelson Interface

Unit Electrical Connection

15 Dec. 95

Unit Mechanical Connection, Rebuild as

Necessary.

15 Dec. 95

5 Oct. 95

15 Dec. 95 Wasson /

15 Dec. 95 MIS

Analytical Equipment Delivery

Installation \& Test

Mar.:

Hazards Review Complete

Leak Check / Operational Test

Apr.:
1 Mar. 96

29 Mar.96

Dahl

Dahl
CRSD/MIS

HT Lyons

Dahl

Wasson

Shake Down Run

3 May 96

Dahl 